\title{
NONSMOOTH SEQUENTIAL ANALYSIS IN ASPLUND SPACES
}

\author{
BORIS S. MORDUKHOVICH AND YONGHENG SHAO
}

\begin{abstract}
We develop a generalized differentiation theory for nonsmooth functions and sets with nonsmooth boundaries defined in Asplund spaces. This broad subclass of Banach spaces provides a convenient framework for many important applications to optimization, sensitivity, variational inequalities, etc. Our basic normal and subdifferential constructions are related to sequential weak-star limits of Fréchet normals and subdifferentials. Using a variational approach, we establish a rich calculus for these nonconvex limiting objects which turn out to be minimal among other set-valued differential constructions with natural properties. The results obtained provide new developments in infinite dimensional nonsmooth analysis and have useful applications to optimization and the geometry of Banach spaces.
\end{abstract}

\section{INTRODUCTION}

It is well known that nonsmooth functions, sets with nonsmooth boundaries and set-valued mappings appear naturally and frequently in various areas of mathematics and applications. We refer the reader to [2, 10, 11, 39, 44, 48, 63] for many examples and motivations, especially related to optimization, stability, variational systems, and control systems. A systematic study of the local behavior of nondifferentiable objects is accomplished in the framework of nonsmooth analysis, which has become an active and fruitful area of mathematics, rich with applications. The term "nonsmooth analysis" was coined in the 1970s by Francis Clarke, who performed pioneering work in this area for fairly general nonsmooth objects.

The heart of nonsmooth analysis is the generalized differentiation of nonsmooth functions. Over the last twenty years great progress has been made in the development and applications of workable calculus for various set-valued generalized derivatives; see $[2,5,10,11,24,26,30,39,44,46,49,56,59,63,68,69]$ and references therein. While the subdifferential theory in finite dimensions has been well developed, there still exist many open principal questions in infinite dimensional spaces.

There are several natural ways to define general subdifferentials satisfying useful calculus rules. In particular, it can be done taking limits of more primitive subdifferential constructions which do not possess such a calculus. It is important that limiting constructions depend not only on the choice of primitive objects but also

Received by the editors June 8, 1994 and, in revised form, April 3, 1995.

1991 Mathematics Subject Classification. Primary 49J52; Secondary 46B20, 58C20.

Key words and phrases. Nonsmooth analysis, generalized differentiation, nonconvex calculus, Asplund spaces, variational principles, Fréchet normals and subdifferentials, sequential limits.

This research was partially supported by the National Science Foundation under grants DMS9206989 and DMS-9404128. 
on the character of the limit: topological or sequential. The topological way allows one to develop useful subdifferentials in general infinite dimensional settings, but it may lead to rather complicated and broad constructions. The sequential way usually leads to smaller and more convenient objects, but it requires some special geometric properties of spaces in question; see discussions and examples in Borwein-Fitzpatrick [4].

The sequential nonconvex subdifferential constructions in Banach spaces were first introduced in Kruger-Mordukhovich [34, 35] on the basis of sequential limits of Fréchet $\varepsilon$-normals and subdifferentials. Such limiting normal cone and subdifferential appeared as infinite dimensional extensions of the corresponding finite dimensional constructions in Mordukhovich [41, 42] motivated by applications to optimization and control. Useful properties of those and related constructions were revealed mainly for Banach spaces with Fréchet differentiable renorms; see, e.g., $[5,8,25,29,31,32,33,34,35,38,39,40,44,54,65,67]$ and the subsequent sections for more details.

On the other line of development, a series of infinite dimensional extensions of the nonconvex constructions in [41, 42] were introduced and studied by Ioffe [20, 23, $24]$ on the basis of topological limits of Dini subdifferentials and $\varepsilon$-subdifferentials. Such constructions, called "approximate subdifferentials", are well defined in more general spaces, but all of them (including their "nuclei") may be broader than the Kruger-Mordukhovich extension even for Lipschitz functions on Banach spaces with Fréchet differentiable renorms; see Section 9.

This paper is devoted to the development of sequential subdifferential and related constructions in infinite dimensions, mostly for the class of Banach spaces called Asplund spaces. This class is sufficiently rich and well investigated in the geometric theory of Banach spaces and various applications; see [1, 16, 55] and references therein. In particular, it includes every Banach space with a Fréchet differentiable renorm (hence any reflexive space) and all spaces with separable duals. Moreover, there are Asplund spaces which fail to have even a Gâteaux differentiable renorm.

Here we use the same sequential limits of Fréchet $\varepsilon$-normals and subdifferentials as in $[34,35]$, observing, in addition, that one can let $\varepsilon=0$ in all the formulas for the case of Asplund spaces; see Section 2. For such nonconvex and not topologically closed constructions we develop a comprehensive subdifferential calculus involving lower semicontinuous (l.s.c.) functions defined on arbitrary Asplund spaces. This calculus includes, in particular, sum rules, chain rules, mean value theorems, subdifferentiation of marginal/value functions, etc. Most of the results obtained provide new information for spaces with Fréchet differentiable renorms; many of them are new even for the case of Hilbert spaces, where our constructions coincide with sequential limits of proximal normals/subgradients. Note that a number of important calculus results for the sequential constructions under consideration hold in any Banach spaces, where taking $\varepsilon>0$ in the original limiting formulas is essential; see [52] and corresponding remarks in the text below.

Our approach to subdifferential calculus is mainly based on an extremal principle for set systems that is expressed in terms of Fréchet normals and actually turns out to be an extremal characterization of Asplund spaces; see [51]. This approach is essentially geometric, as well as variational, being related to corresponding generalizations of the classical separation theorem to the case of nonconvex sets. In this way we are able to obtain calculus results for nonconvex sequential subdifferentials of extended-real-valued functions that may not be locally Lipschitzian. The latter 
case includes the calculus of normal cones to closed sets, which is of particular importance for applications to sensitivity analysis; cf. [47, 48, 53].

The remainder of the paper is organized as follows. In Section 2 we present basic definitions and preliminaries needed in the sequel. Here we also obtain equivalent representations of the Kruger-Mordukhovich limiting normal cone and related subdifferential/coderivative constructions in terms of sequential limits of the corresponding Fréchet (not $\varepsilon$-Fréchet) counterparts in Asplund spaces.

In Section 3 we study a concept of extremality for systems of closed sets in Banach spaces which is at the ground of our approach to subdifferential calculus. In the case of Asplund spaces we obtain a necessary condition for extremal points of set systems in terms of Fréchet normals. Under some additional local compactness assumptions we establish necessary extremality conditions in terms of the nonconvex limiting normal cone. These results imply, in particular, a nonconvex analogue of the Bishop-Phelps densitity theorem and its limiting counterpart in Asplund spaces.

Section 4 is devoted to the fundamental calculus results about representing the (limiting) subdifferentials and singular subdifferentials for sums of l.s.c. extendedreal-valued functions defined on Asplund spaces. Based on the extremal principle in Section 3, we prove refined sum rules for those subdifferentials under minimal assumptions. As a corollary we obtain a formula for representing the nonconvex normal cone to the intersection of closed sets.

In Section 5 we consider single-valued continuous mappings between Banach spaces. The main purpose is to establish relationships between the coderivative for such a mapping and the subdifferential of its Lagrange scalarization. We prove the principal scalarization formula in the form of equality for a class of strictly Lipschitzian mappings defined on Asplund spaces.

Section 6 is devoted to the subdifferentiation of general marginal functions that can be viewed as value functions in problems of parametric optimization. In this way we obtain a series of chain rules for the nonconvex sequential subdifferentials of compositions between nonsmooth mappings in Asplund spaces. The chain rules are proved in both inclusion and equality forms under minimal assumptions.

In Section 7 we obtain more calculus rules for the subdifferentiation of maxima, minima, products, and quotients of real-valued functions, as well as for relations between "partial" and "full" subdifferentials for functions of two variables. Using the chain rule in Section 6, we establish a generalization of the classical mean value theorem for continuous functions defined on Asplund spaces.

Section 8 is devoted to an approximate mean value theorem of Zagrodny's type [70] for l.s.c. functions. We prove refined results of this kind in terms of Fréchet subgradients in Asplund spaces and obtain some useful applications, including characterizations of local Lipschitzian behavior and exact relationships between our basic nonconvex limiting objects and Clarke's normal cone and subdifferential.

In the concluding Section 9 we study the interrelation between our basic sequential constructions and Ioffe's constructions of approximate subdifferentials for the case of Asplund spaces. Invoking recent results and examples in Borwein and Fitzpatrick [4], we prove that our sequential subdifferential for l.s.c. functions is included in any of the approximate subdifferentials and their "nuclei", being strictly better even in the case of Lipschitz functions on Fréchet differentiable spaces. Furthermore, the weak-star closures of the sequential objects give exactly the so-called $G$-normal cones and subdifferentials, while Clarke's constructions coincide with 
the weak-star closures of the corresponding convex hulls. We also establish the minimality of the basic sequential subdifferential for l.s.c. functions among any subdifferential constructions satisfying some natural requirements.

Our notation is basically standard. For any Banach space $X$ we denote its norm by $\|\cdot\|$ and consider the dual space $X^{\star}$ equipped with the weak-star topology $w^{\star}$, where $\langle\cdot, \cdot\rangle$ means the canonic pairing. As usual, $B$ and $B^{\star}$ stand for the unit closed balls in the space and dual space in question. The symbol $B_{r}(x)$ denotes the closed ball with center $x$ and radius $r$. Recall that $\mathrm{cl} \Omega$ and co $\Omega$ mean, respectively, the closure and the convex hull of an arbitrary nonempty set $\Omega \subset X$, while the notation $\mathrm{cl}^{\star}$ is used for the weak-star topological closure in $X^{\star}$. The adjoint (dual) operator to a linear continuous operator $A$ is denoted by $A^{\star}$.

In contrast to the case of single-valued mappings $\Phi: X \rightarrow Y$, the symbol $\Phi: X \Rightarrow Y$ stands for a multifunction from $X$ into $Y$. We denote the graph and kernel of $\Phi$ by

$$
\operatorname{gph} \Phi:=\{(x, y) \in X \times Y \mid y \in \Phi(x)\}, \operatorname{ker} \Phi:=\{x \in X \mid 0 \in \Phi(x)\} .
$$

In this paper we often consider multifunctions $\Phi$ from $X$ into the dual space $X^{\star}$. For such objects, the expression

$$
\limsup _{x \rightarrow \bar{x}} \Phi(x)
$$

always means the sequential Kuratowski-Painlevé upper limit with respect to the norm topology in $X$ and the weak-star topology in $X^{\star}$, i.e.,

$$
\begin{aligned}
& \limsup _{x \rightarrow \bar{x}} \Phi(x):=\left\{x^{\star} \in X^{\star} \mid\right. \exists \text { sequences } x_{k} \rightarrow \bar{x} \text { and } x_{k}^{\star} \stackrel{w^{\star}}{\rightarrow} x^{\star} \\
&\text { with } \left.x_{k}^{\star} \in \Phi\left(x_{k}\right) \text { for all } k=1,2, \ldots\right\} .
\end{aligned}
$$

If $\varphi: X \rightarrow \overline{\mathbf{R}}:=[-\infty, \infty]$ is an extended-real-valued function, then, as usual, $\operatorname{dom} \varphi:=\{x \in X$ with $|\varphi(x)|<\infty\}$, epi $\varphi:=\{(x, \mu) \in X \times \mathbf{R} \mid \mu \geq \varphi(x)\}$.

In this case, $\lim \sup \varphi(x)$ and $\lim \inf \varphi(x)$ denote the upper and lower limits of such (scalar) functions in the classical sense. Depending on context, the symbols $x \stackrel{\varphi}{\rightarrow} \bar{x}$ and $x \stackrel{\Omega}{\rightarrow} \bar{x}$ mean, respectively, that $x \rightarrow \bar{x}$ with $\varphi(x) \rightarrow \varphi(\bar{x})$ and $x \rightarrow \bar{x}$ with $x \in \Omega$.

Throughout the paper we use the convention that $a+\emptyset=\emptyset+b=\emptyset$ for any elements $a$ and $b$ and the empty set $\emptyset$. Some special notation will be introduced and explaned in Sections 2 and 9.

\section{BASIC DEFINITIONS AND REPRESENTATIONS}

This section contains preliminary material on the basic generalized differentiability concepts studied in the paper. We also present useful representations of these constructions for the case of Asplund spaces. Developing a geometric approach to the generalized differentiation, let us start with the definitions of normal elements to arbitrary sets in Banach spaces as in Kruger-Mordukhovich [34, 35].

2.1. Definition. Let $\Omega$ be a nonempty subset of the Banach space $X$ and let $\varepsilon \geq 0$.

(i) Given $x \in \operatorname{cl} \Omega$, the nonempty set

$$
\hat{N}_{\varepsilon}(x ; \Omega):=\left\{x^{\star} \in X^{\star} \mid \limsup _{u \rightarrow x} \frac{\left\langle x^{\star}, u-x\right\rangle}{\|u-x\|} \leq \varepsilon\right\}
$$


is called the set of Fréchet $\varepsilon$-normals to $\Omega$ at $x$. When $\varepsilon=0$, the set (2.1) is a cone which is called the Fréchet normal cone to $\Omega$ at $x$ and is denoted by $\hat{N}(x ; \Omega)$. If $x \notin \operatorname{cl} \Omega$, we let $\hat{N}_{\varepsilon}(x ; \Omega)=\emptyset$ for all $\varepsilon \geq 0$.

(ii) Let $\bar{x} \in \operatorname{cl} \Omega$. The nonempty cone

$$
N(\bar{x} ; \Omega):=\limsup _{x \rightarrow \bar{x}, \varepsilon \downarrow 0} \hat{N}_{\varepsilon}(x ; \Omega)
$$

is called the normal cone to $\Omega$ at $\bar{x}$. We let $N(\bar{x} ; \Omega)=\emptyset$ for $\bar{x} \notin \operatorname{cl} \Omega$.

Note that the normal cone (2.2), in constrast to (2.1) for $\varepsilon>0$, does not change if one replaces the norm $\|\cdot\|$ by another equivalent norm in $X$. In the finite dimensional case $X=\mathbf{R}^{n}$, the normal cone (2.2) coincides with the one in Mordukhovich [41]:

$$
N(\bar{x} ; \Omega)=\limsup _{x \rightarrow \bar{x}}[\operatorname{cone}(x-\Pi(x, \Omega))]
$$

where "cone" means the conic hull of a set and $\Pi(x, \Omega)$ is the multi-valued Euclidean projector of $x$ on the closure of $\Omega$.

Let us observe that the set of Fréchet $\varepsilon$-normals (2.1) is convex for any $\varepsilon \geq 0$ while the normal cone (2.2) is nonconvex even in simple finite dimensional situations (e.g., for $\Omega=\operatorname{gph}|x|$ at $\bar{x}=0 \in \mathbf{R}^{2}$ ). If the space $X$ is infinite dimensional, the weakstar topology of $X^{\star}$ is not sequential and the sequential upper limit in (2.2) does not ensure that the set $N(\bar{x} ; \Omega)$ is closed in both norm and weak-star topologies of $X^{\star}$. Recently Fitzpatrick (personal communication) provided such examples in the Hilbert case $X=l^{2}$; cf. also [4]. Nevertheless, this limiting normal cone possesses a much broader spectrum of useful properties in comparison with the Fréchet normal cone $\hat{N}(\bar{x} ; \Omega)$ and its $\varepsilon$-perturbations $(2.1)$; see $[8,11,22,25,26,27,29,31,32$, $33,34,35,38,39,40,41,42,43,44,45,46,47,48,49,50,51,52,53,63,65]$ and references therein for more details and related material.

It follows directly from (2.2) that

$$
\hat{N}(\bar{x} ; \Omega) \subset N(\bar{x} ; \Omega)
$$

for any set $\Omega \subset X$ and any point $\bar{x} \in \operatorname{cl} \Omega$. The set $\Omega$ is called regular at $\bar{x}$ if (2.3) holds as equality.

The class of regular sets includes all convex sets for which

$$
\hat{N}_{\varepsilon}(\bar{x} ; \Omega)=\left\{x^{\star} \in X^{\star} \mid\left\langle x^{\star}, x-\bar{x}\right\rangle \leq \varepsilon\|x-\bar{x}\| \text { for any } x \in \Omega\right\} \quad \forall \varepsilon \geq 0
$$

and both cones in (2.3) coincide with the normal cone of convex analysis. In general, (2.3) does not hold as equality in many common situations important for applications.

In particular, such a regularity is always broken for finite dimensional sets which are nonsmooth Lipschitzian manifolds in the sense of Rockafellar [62], i.e., they can be locally represented as graphs of nonsmooth Lipschitz continuous functions. This class of sets covers graphs of maximal monotone operators and includes subdifferential mappings for convex, concave, and saddle functions; we refer the reader to $[48,62]$ for more details and discussions. It turns out that such graphical sets naturally appear in the following derivative-like constructions for multifunctions which are realizations of the geometric (graphical) approach to differentiation going back to Fermat. 
2.2. Definition. Let $\Phi: X \Rightarrow Y$ be a multifunction between Banach spaces $X$ and $Y$, and let $(\bar{x}, \bar{y}) \in \operatorname{cl}$ gph $\Phi$. The multifunction $D^{\star} \Phi(\bar{x}, \bar{y})$ from $Y^{\star}$ into $X^{\star}$ defined by

$$
D^{\star} \Phi(\bar{x}, \bar{y})\left(y^{\star}\right):=\left\{x^{\star} \in X^{\star} \mid\left(x^{\star},-y^{\star}\right) \in N((\bar{x}, \bar{y}) ; \text { gph } \Phi)\right\}
$$

is called the coderivative of $\Phi$ at $(\bar{x}, \bar{y})$. The symbol $D^{\star} \Phi(\bar{x})\left(y^{\star}\right)$ is used in (2.4) when $\Phi$ is single-valued at $\bar{x}$ and $\bar{y}=\Phi(\bar{x})$. We let $D^{\star} \Phi(\bar{x}, \bar{y})\left(y^{\star}\right)=\emptyset$ if $(\bar{x}, \bar{y}) \notin \operatorname{cl} \operatorname{gph} \Phi$.

In the finite dimensional setting the coderivative (2.4) was introduced and studied in $[42,44,49]$. Defining by analogy the Fréchet $\varepsilon$-coderivative as

$$
\hat{D}_{\varepsilon}^{\star} \Phi(\bar{x}, \bar{y})\left(y^{\star}\right):=\left\{x^{\star} \in X^{\star} \mid\left(x^{\star},-y^{\star}\right) \in \hat{N}_{\varepsilon}((\bar{x}, \bar{y}) ; \operatorname{gph} \Phi)\right\} \quad \forall \varepsilon \geq 0,
$$

one has the limiting representation

$$
D^{\star} \Phi(\bar{x}, \bar{y})\left(\bar{y}^{\star}\right)=\limsup _{\left(x, y, y^{\star}\right) \rightarrow\left(\bar{x}, \bar{y}, \bar{y}^{\star}\right), \varepsilon \downarrow 0} \hat{D}_{\varepsilon}^{\star} \Phi(x, y)\left(y^{\star}\right) .
$$

When $\varepsilon=0$, the construction (2.5) is called the Fréchet coderivative of $\Phi$ at $(\bar{x}, \bar{y})$ and is denoted by $\hat{D}^{\star} \Phi(\bar{x}, \bar{y})$. If both spaces $X$ and $Y$ are reflexive, the Fréchet coderivative is dual to a graphical derivative object (in the vein of Aubin-Frankowska [2, Chapter 5]) generated by the "weak contingent cone". On the contrary, the coderivative (2.4) is not convex-valued and is not dual to any tangentially generated derivative-type object for multifunctions.

Let $\Phi$ be locally single-valued around $\bar{x}$. Recall that $\Phi: X \rightarrow Y$ is said to be strictly differentiable at $\bar{x}$ with the derivative $\Phi^{\prime}(\bar{x})$ if

$$
\lim _{x \rightarrow \bar{x}, u \rightarrow \bar{x}} \frac{\Phi(x)-\Phi(u)-\Phi^{\prime}(\bar{x})(x-u)}{\|x-u\|}=0 .
$$

It is well known that any mapping $\Phi$ continuously Fréchet differentiable around $\bar{x}$ is strictly differentiable at $\bar{x}$, but not vice versa. The next proposition can be derived directly from the definitions; cf. [44, 50].

2.3. Proposition. Let $X$ and $Y$ be Banach spaces and let $\Phi: X \rightarrow Y$ be strictly differentiable at $\bar{x}$. Then

$$
D^{\star} \Phi(\bar{x})\left(y^{\star}\right)=\hat{D}^{\star} \Phi(\bar{x})\left(y^{\star}\right)=\left(\Phi^{\prime}(\bar{x})\right)^{\star} y^{\star} \forall y^{\star} \in Y^{\star} .
$$

Thus the coderivative (2.4) is a proper set-valued generalization of the adjoint linear operator to the classical strict derivative. In Section 5 we obtain a useful representation of the coderivative (2.4) for the class of nonsmooth strictly Lipschitzian mappings defined on Asplund spaces. In that formula we express the coderivative of a mapping with values in a Banach space in terms of the following subdifferential construction for its Lagrange scalarization.

Let $\varphi: X \rightarrow \overline{\mathbf{R}}$ be an extended-real-valued function defined on a Banach space and let

$$
\Phi(x)=E_{\varphi}(x):=\{\mu \in \mathbf{R} \mid \mu \geq \varphi(x)\}
$$

be the associated epigraphical multifunction with gph $\Phi=$ epi $\varphi$. Following the line in $[41,34,44]$, we define the basic subdifferential constructions geometrically as special cases of the coderivative (2.4) related to the normal cone (2.2).

2.4. Definition. Let $\bar{x} \in \operatorname{dom} \varphi$. The sets

$$
\partial \varphi(\bar{x}):=D^{\star} E_{\varphi}(\bar{x}, \varphi(\bar{x}))(1)=\left\{x^{\star} \in X^{\star} \mid\left(x^{\star},-1\right) \in N((\bar{x}, \varphi(\bar{x})) ; \text { epi } \varphi)\right\},
$$


(2.8) $\partial^{\infty} \varphi(\bar{x}):=D^{\star} E_{\varphi}(\bar{x}, \varphi(\bar{x}))(0)=\left\{x^{\star} \in X^{\star} \mid\left(x^{\star}, 0\right) \in N((\bar{x}, \varphi(\bar{x}))\right.$; epi $\left.\varphi)\right\}$

are called, respectively, the subdifferential and the singular subdifferential of $\varphi$ at $\bar{x}$. We let $\partial \varphi(\bar{x})=\partial^{\infty} \varphi(\bar{x})=\emptyset$ if $\bar{x} \notin \operatorname{dom} \varphi$.

The subdifferential (2.7) generalizes the concept of strict derivative to the case of nonsmooth functions and is reduced to the subdifferential of convex analysis if $\varphi$ is convex. It follows from (2.1) and (2.2) that for functions $\varphi$ l.s.c. around $\bar{x}$, the subdifferential (2.7) can be represented in the limiting form

$$
\partial \varphi(\bar{x})=\lim _{x \rightarrow \bar{\varphi}} \sup \hat{\partial}_{\varepsilon} \varphi(x)
$$

in terms of the Fréchet $\varepsilon$-subdifferentials

$$
\hat{\partial}_{\varepsilon} \varphi(x):=\hat{D}_{\varepsilon}^{\star} E_{\varphi}(x, \varphi(x))(1)=\left\{x^{\star} \in X^{\star} \mid\left(x^{\star},-1\right) \in \hat{N}_{\varepsilon}((x, \varphi(x)) ; \text { epi } \varphi)\right\},
$$

where $x \in \operatorname{dom} \varphi, \varepsilon \geq 0$ with $\hat{\partial}_{\varepsilon} \varphi(x)=\emptyset$ for $x \notin \operatorname{dom} \varphi$. If $\varepsilon=0$ in (2.10), this set is called the Fréchet subdifferential of $\varphi$ at $x$ and is denoted by $\hat{\partial} \varphi(x)$.

It follows from $(2.9)$ that $\hat{\partial} \varphi(\bar{x}) \subset \partial \varphi(\bar{x})$. The function $\varphi$ is called subdifferentially regular at $\bar{x}$ if the latter holds as equality. The class of subdifferentially regular functions includes all convex functions, smooth (strictly differentiable) functions, "max functions", etc. On the other hand, such a unilateral regularity fails, in particular, for nonsmooth concave functions like $\varphi(x)=-|x|, x \in \mathbf{R}$, where $\partial \varphi(0)=\{-1,1\}$ but $\hat{\partial} \varphi(0)=\emptyset$.

In some situations it is more convenient to replace $\hat{\partial}_{\varepsilon} \varphi(x)$ in $(2.9)$ by its analytic counterpart

$$
\tilde{\partial}_{\varepsilon} \varphi(x):=\left\{x^{\star} \in X^{\star} \mid \liminf _{u \rightarrow x} \frac{\varphi(u)-\varphi(x)-\left\langle x^{\star}, u-x\right\rangle}{\|u-x\|} \geq-\varepsilon\right\}, \quad \varepsilon \geq 0,
$$

which is effectively used, in particular, in the theory of viscosity solutions [15]. This follows from a fact proved in Kruger [31, Theorem 1] and Ioffe [25, Proposition 1]: $\tilde{\partial}_{\varepsilon} \varphi(x) \subset \hat{\partial}_{\varepsilon} \varphi(x)$ and

$$
\left[x^{\star} \in \hat{\partial}_{\varepsilon} \varphi(x)\right] \Longrightarrow\left[x^{\star} \in \tilde{\partial}_{\tilde{\varepsilon}} \varphi(x)\right] \text { for } \tilde{\varepsilon}:=(\varepsilon /(1-\varepsilon))\left(1+\left\|x^{\star}\right\|\right)
$$

when $\varphi$ is l.s.c. at $x$. Therefore, $\tilde{\partial}_{0} \varphi(x)=\hat{\partial} \varphi(x)$, and one has

$$
\partial \varphi(\bar{x})=\lim _{x \rightarrow \bar{\varphi}} \sup _{x \downarrow} \tilde{\partial}_{\varepsilon} \varphi(x)
$$

for any function $\varphi: X \rightarrow \overline{\mathbf{R}}$ l.s.c. around $\bar{x}$.

Regarding the singular subdifferential (2.8), we observe that this construction makes sense only for non-Lipschitzian functions. Indeed, directly from the definitions one can derive the following assertion; see [52].

2.5. Proposition. Let $X$ be a Banach space and let $\varphi: X \rightarrow \overline{\mathbf{R}}$ be Lipschitz continuous around $\bar{x}$. Then $\partial^{\infty} \varphi(\bar{x})=\{0\}$.

Further, one can easily check that the normal constructions (2.1) and (2.2) to any set $\Omega \subset X$ at $\bar{x} \in \Omega$ are expressed in the subdifferential forms

$$
\begin{gathered}
\hat{N}_{\varepsilon}(\bar{x} ; \Omega)=\tilde{\partial}_{\varepsilon} \delta(\bar{x}, \Omega) \text { for any } \varepsilon \geq 0, \\
N(\bar{x} ; \Omega)=\partial \delta(\bar{x}, \Omega)=\partial^{\infty} \delta(\bar{x}, \Omega),
\end{gathered}
$$


where $\delta(\cdot, \Omega)$ is the indicator function of $\Omega$, that is, $\delta(x, \Omega)=0$ if $x \in \Omega$ and $\delta(x, \Omega)=\infty$ if $x \notin \Omega$.

On the other hand, the normal cone (2.2) admits a useful representation in terms of the subdifferential (2.7) of the Lipschitz continuous distance function

$$
\operatorname{dist}(x, \Omega):=\inf \{\|x-\omega\| \text { s.t. } \omega \in \Omega\}
$$

to the set $\Omega$. The following result can be deduced from Thibault [65, Proposition $2.7]$.

2.6. Proposition. Let $\Omega$ be a nonempty subset of a Banach space $X$. Then

$$
N(\bar{x} ; \Omega)=\bigcup_{\lambda>0} \lambda \partial \operatorname{dist}(\bar{x}, \Omega) \forall \bar{x} \in \operatorname{cl} \Omega .
$$

All the results discussed above are valid in any Banach spaces. Now let us assume the spaces considered are Asplund [1], i.e., they are Banach spaces on which every continuous convex function is Fréchet differentiable at a dense set of points. We refer the reader to the recent books $[16,55]$ for various properties and characterizations of such spaces that turn out to be very important for theory and applications. One of the most useful characterizations is as follows: a Banach space is Asplund if and only if each of its separable subspaces has a separable dual.

Note that the class of Asplund spaces turns out to be essentially broader than the class of spaces with Fréchet differentiable renorms. According to Haydon's examples (see [16, Chapter 7]), there are Asplund spaces of continuous functions where every equivalent norm fails to be even Gâteaux differentiable at some nonzero points. One can find many interesting subclasses and examples of Asplund spaces in $[16,55]$.

In this paper we will use the following "fuzzy sum rule" for Fréchet subdifferentials in Asplund spaces, proved by Fabian [18, Theorem 3] with the help of the Borwein-Preiss smooth variational principle [6] and a separable reduction.

2.7. Proposition. Let $X$ be an Asplund space and let $\varphi_{i}: X \rightarrow \overline{\mathbf{R}}, i=1,2$, be l.s.c. functions one of which is Lipschitz continuous around $\bar{x} \in \operatorname{dom} \varphi_{1} \cap \operatorname{dom} \varphi_{2}$. Then for any $\varepsilon \geq 0, \delta>0$, and $\gamma>0$ one has

$$
\begin{aligned}
\tilde{\partial}_{\varepsilon}\left(\varphi_{1}+\varphi_{2}\right)(\bar{x}) \subset \bigcup\left\{\hat{\partial} \varphi_{1}\left(x_{1}\right)+\hat{\partial} \varphi_{2}\left(x_{2}\right) \mid x_{i} \in B_{\delta}(\bar{x}),\right. \\
\left.\left|\varphi_{i}\left(x_{i}\right)-\varphi_{i}(\bar{x})\right| \leq \delta, i=1,2\right\}+(\varepsilon+\gamma) B^{\star} .
\end{aligned}
$$

Applying this result for the case of $\varphi_{1}=\varphi$ and $\varphi_{2}=0$, we get

2.8. Corollary. Let $X$ be an Asplund space, let $\varphi: X \rightarrow \overline{\mathbf{R}}$ be a function l.s.c. around $\bar{x} \in \operatorname{dom} \varphi$, and let $\varepsilon \geq 0, \delta>0, \gamma>0$. Then

$$
\tilde{\partial}_{\varepsilon} \varphi(\bar{x}) \subset \bigcup\left\{\hat{\partial} \varphi(x)\left|x \in B_{\delta}(\bar{x}),\right| \varphi(x)-\varphi(\bar{x}) \mid \leq \delta\right\}+(\varepsilon+\gamma) B^{\star} .
$$

Now we use Fabian's results to prove refined representations of the normal cone (2.2) as well as the related coderivative and subdifferential constructions (2.4), (2.7), and (2.8). The representations obtained extend corresponding results of Ioffe [25] from spaces with Fréchet differentiable renorms to the general case of Asplund spaces.

2.9. Theorem. Let $X$ and $Y$ be Asplund spaces. Then the following hold: 
(i) For any set $\Omega \subset X$ and any $\bar{x} \in \operatorname{cl} \Omega$ one has

$$
N(\bar{x} ; \Omega)=\limsup _{x \rightarrow \bar{x}} \hat{N}(x ; \Omega) .
$$

(ii) For any multifunction $\Phi: X \Rightarrow Y$ and any $(\bar{x}, \bar{y}) \in \operatorname{clgph} \Phi$ one has

$$
D^{\star} \Phi(\bar{x}, \bar{y})\left(\bar{y}^{\star}\right)=\limsup _{\left(x, y, y^{\star}\right) \rightarrow\left(\bar{x}, \bar{y}, \bar{y}^{\star}\right)} \hat{D}^{\star} \Phi(x, y)\left(y^{\star}\right) .
$$

(iii) For any function $\varphi: X \rightarrow \overline{\mathbf{R}}$ l.s.c. around $\bar{x} \in \operatorname{dom} \varphi$ one has

$$
\begin{aligned}
& \partial \varphi(\bar{x})=\limsup _{x_{\varphi}^{\varphi} \bar{x}} \hat{\partial} \varphi(x), \\
& \partial^{\infty} \varphi(\bar{x})=\lim _{x \stackrel{\varphi}{\rightarrow} \bar{x} ; \varepsilon, \lambda \downarrow 0} \lambda \hat{\partial}_{\varepsilon} \varphi(x)=\lim _{x \stackrel{\varphi}{\rightarrow} \bar{x} ; \varepsilon, \lambda \downarrow 0} \lambda \tilde{\partial}_{\varepsilon} \varphi(x)=\lim _{x \stackrel{\varphi}{\rightarrow} \bar{x}, \lambda \downarrow 0} \lambda \hat{\partial} \varphi(x) .
\end{aligned}
$$

Proof. Let us prove (i). By virtue of the normal cone definition (2.2), it suffices to verify that $N(\bar{x} ; \Omega)$ is contained in the right-hand side of $(2.15)$. Taking any $x^{\star} \in N(\bar{x} ; \Omega)$, we can find sequences $x_{k} \rightarrow \bar{x}, x_{k}^{\star} \stackrel{w^{\star}}{\rightarrow} x^{\star}$, and $\varepsilon_{k} \downarrow 0$ such that $x_{k} \in \Omega$ and $x_{k}^{\star} \in \hat{N}_{\varepsilon_{k}}\left(x_{k} ; \Omega\right)$ for $k=1,2, \ldots$ Due to (2.13) the latter implies that $x_{k}^{\star} \in \tilde{\partial}_{\varepsilon_{k}} \delta\left(x_{k}, \Omega\right)$, where one may consider $\varepsilon_{k}>0$ for all $k$.

Now let us apply Corollary 2.8 to the indicator function $\varphi(x)=\delta(x, \Omega)$ at the point $x_{k}$ for each $k$. Using this result for $\varepsilon=\delta=\gamma=\varepsilon_{k}$, we get $\tilde{x}_{k} \in B_{\varepsilon_{k}}\left(x_{k}\right) \cap \Omega$ and $\tilde{x}_{k}^{\star} \in \hat{\partial} \delta\left(\tilde{x}_{k}, \Omega\right)$ such that $\left\|\tilde{x}_{k}^{\star}-x_{k}^{\star}\right\| \leq 2 \varepsilon_{k}$ for $k=1,2, \ldots$ Therefore, $\tilde{x}_{k} \rightarrow \bar{x}$ and $\tilde{x}_{k}^{\star} \stackrel{w^{\star}}{\rightarrow} x^{\star}$ as $k \rightarrow \infty$. Again employing (2.13), we conclude that $\tilde{x}_{k}^{\star} \in \hat{N}\left(\tilde{x}_{k} ; \Omega\right)$, which implies (2.15).

Assertion (ii) immediately follows from (2.15) with $\Omega=$ gph $\Phi$, Definition 2.2, and (2.5) as $\varepsilon=0$. In turn, this implies (2.16) for l.s.c. functions by virtue of $(2.7)$ and (2.10). Note that (2.16) can be obtained directly from (2.9) if we use Corollary 2.8. It remains to prove (2.17) for the singular subdifferentials in Asplund space. To verify this it suffices to show that

$$
\partial^{\infty} \varphi(\bar{x}) \subset \lim _{x \rightarrow \bar{x}} \sup _{x \downarrow \lambda} \lambda \hat{\partial} \varphi(x)
$$

since the other inclusions in (2.17) follow directly from the definitions.

Let $x^{\star} \in \partial^{\infty} \varphi(\bar{x})$, i.e., $\left(x^{\star}, 0\right) \in N((\bar{x}, \varphi(\bar{x}))$; epi $\varphi)$. It follows from assertion (i) of this theorem that there exist $\left(x_{k}, r_{k}\right) \stackrel{e p i \varphi}{\rightarrow}(\bar{x}, \varphi(\bar{x}))$ and $\left(x_{k}^{\star},-\varepsilon_{k}\right) \stackrel{w^{\star}}{\rightarrow}\left(x^{\star}, 0\right)$ as $k \rightarrow \infty$ such that $\left(x_{k}^{\star},-\varepsilon_{k}\right) \in \hat{N}\left(\left(x_{k}, r_{k}\right)\right.$; epi $\left.\varphi\right)$ for all $k=1,2, \ldots$ The latter implies that $\varepsilon_{k} \geq 0$ for all $k$. Thus one has two possiblities for the sequence $\left\{\left(x_{k}^{\star}, \varepsilon_{k}\right)\right\}$ : either

(a) there exists a subsequence of $\left\{\varepsilon_{k}\right\}$ consisting of positive numbers, or

(b) $\varepsilon_{k}=0$ for all $k$ sufficiently large.

In the case (a) we assume, without loss of generality, that $\varepsilon_{k}>0$ for all $k$, which implies $r_{k}=\varphi\left(x_{k}\right)$ and $x_{k}^{\star} / \varepsilon_{k} \in \hat{\partial} \varphi\left(x_{k}\right)$ for $k=1,2, \ldots$ Hence, letting $\lambda_{k}:=\varepsilon_{k}$ and $\tilde{x}_{k}^{\star}:=x_{k}^{\star} / \varepsilon_{k}$, we get $\lambda_{k} \tilde{x}_{k}^{\star} \stackrel{w^{\star}}{\rightarrow} x^{\star}$ and $\lambda \downarrow 0$ as $k \rightarrow \infty$.

In the case (b), one always has $\left(x_{k}^{\star}, 0\right) \in \hat{N}\left(\left(x_{k}, \varphi\left(x_{k}\right)\right)\right.$; epi $\left.\varphi\right)$. Now using Proposition 2.7 and the technique developed in Ioffe [25, proof of Theorem 4], we get sequences $\left\{\tilde{x}_{k}\right\},\left\{\tilde{x}_{k}^{\star}\right\}$, and $\left\{\lambda_{k}\right\}$ such that

$$
\left(\tilde{x}_{k}^{\star},-1\right) \in \hat{N}\left(\left(\hat{x}_{k}, \varphi\left(\hat{x}_{k}\right)\right) ; \text { epi } \varphi\right), \quad \hat{x}_{k} \stackrel{\varphi}{\rightarrow} \bar{x}, \quad \lambda_{k} \downarrow 0, \text { and } \lambda_{k} \tilde{x}_{k} \stackrel{w^{\star}}{\rightarrow} x^{\star} \text { as } k \rightarrow \infty \text {. }
$$


This establishes (2.18) and completes the proof of the theorem.

2.10. Remark. Equalities (2.17) provide convenient representations of the singular subdifferential (2.8) that is widely used in this paper. They go back to the corresponding Rockafellar theorem in finite dimensions [59]. Note that the intermediate limits in (2.17) are equal in any Banach space, due to the relationship between (2.10) and (2.11) mentioned above. Such constructions possess some useful properties in the general Banach space setting; see $[52,65]$.

To conclude this section let us consider the $\varepsilon$-subdifferential construction

$$
\partial_{\varepsilon} \varphi(\bar{x}):=\limsup _{x \stackrel{\varphi}{\rightarrow} \bar{x}} \tilde{\partial}_{\varepsilon} \varphi(x), \quad \varepsilon \geq 0
$$

where $\tilde{\partial}_{\varepsilon} \varphi(x)$ is defined in (2.11). Construction (2.19) was recently introduced in [28] and effectively employed therein to study relationships between concepts of $\varepsilon$-convexity for extended-real-valued functions and $\varepsilon$-monotonicity for their subdifferential mappings. It follows from Theorem 2.9(iii) that when $\varepsilon=0$ the set (2.19) coincides with our basic subdifferential (2.7) for l.s.c. functions in Asplund spaces. Let us establish the link between (2.7) and (2.19) for any $\varepsilon \geq 0$ that we observed after receiving [28].

2.11. Proposition. Let $X$ be an Asplund space and let $\varphi: X \rightarrow \overline{\mathbf{R}}$ be l.s.c. around $\bar{x} \in \operatorname{dom} \varphi$. Then

$$
\partial_{\varepsilon} \varphi(\bar{x})=\partial \varphi(\bar{x})+\varepsilon B^{\star}
$$

for all $\varepsilon \geq 0$.

Proof. The inclusion " $\supset$ " in (2.20) is trivial. Let us prove the opposite inclusion. Consider any $x^{\star} \in \partial_{\varepsilon} \varphi(\bar{x})$. Due to the definition (2.19) there exist sequences $x_{k} \stackrel{\varphi}{\rightarrow} \bar{x}$ and $x_{k}^{\star} \stackrel{w^{\star}}{\rightarrow} x^{\star}$ as $k \rightarrow \infty$ such that $x_{k}^{\star} \in \tilde{\partial}_{\varepsilon} \varphi\left(x_{k}\right)$ for all $k=1,2, \ldots$ Picking any sequence $\delta_{k} \downarrow 0$ as $k \rightarrow \infty$ and employing Corollary 2.8 with $\delta=\gamma=\delta_{k}$, we find $u_{k} \in x_{k}+\delta_{k} B$ such that $\left|\varphi\left(u_{k}\right)-\varphi\left(x_{k}\right)\right| \leq \delta_{k}$ and

$$
x_{k}^{\star} \in \hat{\partial} \varphi\left(u_{k}\right)+\left(\varepsilon+\delta_{k}\right) B^{\star} \text { for all } k=1,2, \ldots .
$$

This allows us to find $u_{k}^{\star} \in \hat{\partial} \varphi\left(u_{k}\right)$ and $z_{k}^{\star} \in\left(\varepsilon+\delta_{k}\right) B^{\star}$ such that

$$
x_{k}^{\star}=u_{k}^{\star}+z_{k}^{\star} \text { for all } k=1,2, \ldots .
$$

Now taking into account that $X$ is Asplund and the dual unit ball $B^{\star}$ is weak-star sequentially compact in $X^{\star}$, we may assume that

$$
z_{k}^{\star} \stackrel{w^{\star}}{\rightarrow} z^{\star} \text { as } k \rightarrow \infty \text { with }\left\|z^{\star}\right\| \leq \liminf _{k \rightarrow \infty}\left\|z_{k}^{\star}\right\| \leq \varepsilon .
$$

Due to (2.21) the latter yields $u_{k}^{\star} \stackrel{w^{\star}}{\rightarrow} u^{\star}$ as $k \rightarrow \infty$, where $u^{\star} \in \partial \varphi(\bar{x})$ by virtue of (2.16). Now passing to the limit in (2.21) as $k \rightarrow \infty$, we arrive at

$$
\partial_{\varepsilon} \varphi(\bar{x}) \subset \partial \varphi(\bar{x})+\varepsilon B^{\star} .
$$

This ends the proof of the proposition. 
Therefore, all the calculus results obtained below for our basic subdifferential construction (2.7) can be tranfered to the $\varepsilon$-subdifferential (2.19).

\section{EXTREMAL PRINCIPLE}

In this section we study a geometric concept of local extremality for systems of sets and obtain necessary conditions for such an extremality in terms of Fréchet normals, $\varepsilon$-normals, and their sequential limits in Asplund spaces. The results obtained in this way are versions of the extremal principle which is at the heart of our approach to the generalized differential calculus of the nonconvex constructions (2.2), (2.7), and (2.8).

3.1. Definition. Let $\Omega_{1}$ and $\Omega_{2}$ be closed sets in a Banach space $X$ and let $\bar{x} \in$ $\Omega_{1} \cap \Omega_{2}$. Then $\bar{x}$ is called a locally extremal point of the set system $\left\{\Omega_{1}, \Omega_{2}\right\}$ if there are a neighborhood $U$ of $\bar{x}$ and sequences $\left\{a_{i k}\right\} \subset X, i=1,2$, such that $a_{i k} \rightarrow 0$ for $i=1,2$ and

$$
\left(\Omega_{1}-a_{1 k}\right) \cap\left(\Omega_{2}-a_{2 k}\right) \cap U=\emptyset \quad \forall k=1,2, \ldots .
$$

We say that the sets $\Omega_{1}$ and $\Omega_{2}$ generate an extremal system $\left\{\Omega_{1}, \Omega_{2}\right\}$ if they have at least one locally extremal point.

This geometric concept of set extremality $[34,35]$ means that two closed sets with a common point can be locally pushed apart by small perturbations. It covers the usual notions of solution to standard as well as nonstandard problems of scalar and/or vector optimization; see [32, 33, 34, 35, 42, 44]. For example, let $\bar{x}$ be a local optimal solution to the problem

$$
\text { minimize } \varphi(x) \text { subject to } x \in \Omega \text {, }
$$

where $\varphi$ is an extended-real-valued function l.s.c. around $\bar{x}$ and $\Omega$ is a closed set in a Banach space $X$. Then one can see that $(\bar{x}, \varphi(\bar{x}))$ is a locally extremal point of the set system $\left\{\Omega_{1}, \Omega_{2}\right\}$ in $X \times \mathbf{R}$ with $\Omega_{1}=\operatorname{epi} \varphi$ and $\Omega_{2}=\Omega \times\{\varphi(\bar{x})\}$. For checking this, we take $a_{1 k}=\left(0, \nu_{k}\right)$ with $\nu_{k}<0, a_{2 k}=0$ for any $k=1,2, \ldots$, and $U=V \times \mathbf{R}$ in (3.1), where $V$ is a neighborhood of the local minimizer $\bar{x}$ in (3.2).

Another important example of an extremal system is provided by the pair $\{\bar{x}, \Omega\}$ where $\bar{x}$ is a boundary point of the closed set $\Omega \subset X$. There are also close connections between extremality and separability of systems of convex and nonconvex sets; see [44, Section 6].

Now we present necessary conditions for extremal points of closed set systems in Asplund spaces. These conditions can be viewed as generalized Euler equations in the abstract geometric setting expressed in terms of Fréchet normals and $\varepsilon$-normals.

3.2. Theorem. Let $X$ be an Asplund space, let $\Omega_{1}$ and $\Omega_{2}$ be closed sets in $X$, and let $\bar{x} \in \Omega_{1} \cap \Omega_{2}$ be a locally extremal point of the system $\left\{\Omega_{1}, \Omega_{2}\right\}$. Then one has the following two equivalent conditions:

(i) For any $\varepsilon>0$ there exist $x_{i \varepsilon} \in \Omega_{i} \cap B_{\varepsilon}(\bar{x})$ and $x_{i \varepsilon}^{\star} \in \hat{N}_{\varepsilon}\left(x_{i \varepsilon} ; \Omega_{i}\right), i=1,2$, such that

$$
\left\|x_{1 \varepsilon}^{\star}\right\|+\left\|x_{2 \varepsilon}^{\star}\right\|=1 \text { and } x_{1 \varepsilon}^{\star}+x_{2 \varepsilon}^{\star}=0 .
$$

(ii) For any $\varepsilon>0$ there exist $x_{i \varepsilon} \in \Omega_{i} \cap B_{\varepsilon}(\bar{x})$ and $x_{i \varepsilon}^{\star} \in \hat{N}\left(x_{i \varepsilon} ; \Omega_{i}\right)+\varepsilon B^{\star}, i=$ 1,2 , such that relationships (3.3) hold. 
Proof. Developing a classical line in optimization theory, we derive necessary conditions for locally extremal points of set systems from a corresponding open mapping principle. Indeed, one can conclude directly from the definitions that $\bar{x}$ is not a locally extremal point of the system $\left\{\Omega_{1}, \Omega_{2}\right\}$ if the mapping $\Phi: \Omega_{1} \times \Omega_{2} \rightarrow X$ defined by

$$
\Phi(z):=x_{2}-x_{1} \text { for } z=\left(x_{1}, x_{2}\right) \in \Omega:=\Omega_{1} \times \Omega_{2}
$$

possesses the following covering property: there exist a number $a>0$ and a neighborhood $V$ of $\bar{z}:=(\bar{x}, \bar{x})$ such that

$$
B_{a r}(\Phi(z)) \subset \Phi\left(B_{r}(z) \cap \Omega\right) \text { for any }(z, r) \text { with } B_{r}(z) \subset V .
$$

Therefore, any sufficient condition for the covering of mapping (3.4) around $\bar{z}$ generates a necessary condition for the locally extremal point $\bar{x}$ of the system $\left\{\Omega_{1}, \Omega_{2}\right\}$.

In [50], we proved several necessary and sufficient conditions for the covering of multifunctions between Asplund spaces. The following one is more convenient to be employed in the present setting: $\Phi$ enjoys the covering property around $\bar{z}$ if and only if there exists $\varepsilon>0$ such that

$$
\text { ker } \hat{D}_{\varepsilon}^{\star} \Phi(z) \subset B^{\star} \forall x \in B_{\varepsilon}(\bar{z}) \cap \Omega \text {. }
$$

Using criterion (3.5) for the case of mapping (3.4) and taking into account the previous discussions, we can conclude that if $\bar{x}$ is an extremal point of $\left\{\Omega_{1}, \Omega_{2}\right\}$, then for any $\varepsilon>0$ there exist $x_{1 \varepsilon} \in \Omega_{1} \cap B_{\varepsilon}(\bar{x}), x_{2 \varepsilon} \in \Omega_{2} \cap B_{\varepsilon}(\bar{x})$, and $x_{\varepsilon}^{\star} \in$ ker $\hat{D}_{\varepsilon}^{\star} \Phi\left(x_{1 \varepsilon}, x_{2 \varepsilon}\right)$ with $\left\|x_{\varepsilon}^{\star}\right\|>1$. By virtue of (2.5), (2.1), and (3.4) one has

$$
\limsup _{\left(x_{1}, x_{2}\right) \stackrel{\Omega_{1} \times \Omega_{2}}{\rightarrow}\left(x_{1 \varepsilon}, x_{2 \varepsilon}\right)} \frac{-\left\langle x_{\varepsilon}^{\star},\left(x_{2}-x_{1}\right)-\left(x_{2 \varepsilon}-x_{1 \varepsilon}\right)\right\rangle}{\left\|\left(x_{1}, x_{2}\right)-\left(x_{1 \varepsilon}, x_{2 \varepsilon}\right)\right\|} \leq \varepsilon .
$$

Now denoting $x_{1 \varepsilon}^{\star}:=x_{\varepsilon}^{\star} /\left(2\left\|x_{\varepsilon}^{\star}\right\|\right)$ and $x_{2 \varepsilon}^{\star}:=-x_{\varepsilon}^{\star} /\left(2\left\|x_{\varepsilon}^{\star}\right\|\right)$, we get (3.3) and the inclusions $x_{i \varepsilon}^{\star} \in \hat{N}_{\varepsilon}\left(x_{i \varepsilon} ; \Omega_{i}\right), i=1,2$, which follow from (3.6). This implies the first necessary extremality condition of the theorem.

Conclusion (ii) of the theorem follows from (i) by virtue of Corollary 2.8 applied to the indicator functions $\delta\left(\cdot, \Omega_{1}\right)$ and $\delta\left(\cdot, \Omega_{2}\right)$. The opposite implication (ii) $\Longrightarrow(\mathrm{i})$ holds for any Banach space due to the obvious inclusion

$$
\hat{N}(x ; \Omega)+\varepsilon B^{\star} \subset \hat{N}_{\varepsilon}(x ; \Omega) \forall \varepsilon \geq 0
$$

that is valid in the general case. This ends the proof of the theorem.

3.3. Remarks. (a) Condition (i) of Theorem 3.2 was first proved by Kruger and Mordukhovich [34, 35] by using Ekeland's variational principle [17] for spaces with Fréchet differentiable renorms; see also [32, 44]. In [25], Ioffe proved condition (ii) in the Fréchet differentiable setting by using the smooth variational principle of Borwein and Preiss [6] instead of Ekeland's. Our result shows that both extremality conditions (i) and (ii) are valid and equivalent in any Asplund space.

(b) Another proof of Theorem 3.2 can be found in Mordukhovich-Shao [51]. Moreover, in [51] we show that a Banach space $X$ is Asplund if and only if conditions (i) and (ii) are equivalent and hold for any extremal set system in $X$.

The extremal point characteristics obtained in Theorem 3.2 lead to necessary optimality conditions for general problems of nonsmooth optimization in Asplund spaces; this topic is beyond the scope of the present paper. On the other hand, employing those characteristics, one can easily get some useful consequences related to 
the geometry of Banach spaces. The following result may be viewed as a nonconvex generalization of the celebrated Bishop-Phelps density theorem in terms of Fréchet normals in Asplund spaces; cf. [5, 8, 55].

3.4. Corollary. Let $\Omega \subset X$ be a nonempty closed subset of an Asplund space $X$. Then the set of points

$$
x \in \Omega \text { with } \hat{N}(x ; \Omega) \neq\{0\}
$$

is dense in the boundary of $\Omega$.

Proof. Let $\bar{x}$ be a boundary point of the set $\Omega$. Then it is a locally extremal point of the system $\left\{\Omega_{1}, \Omega_{2}\right\}$, where $\Omega_{1}:=\Omega$ and $\Omega_{2}:=\{\bar{x}\}$. Let us apply to this case the necessary extremality condition (ii) of Theorem 3.2 with $0<\varepsilon \leq 1 / 2$. Using this result, we find $x_{\varepsilon} \in \Omega$ and $x_{1 \varepsilon}^{\star}, x_{2 \varepsilon}^{\star} \in X^{\star}$ such that

$$
x_{\varepsilon} \in B_{\varepsilon}(\bar{x}), \quad x_{1 \varepsilon}^{\star} \in \hat{N}\left(x_{\varepsilon} ; \Omega\right)+\varepsilon B^{\star}, \quad\left\|x_{1 \varepsilon}^{\star}\right\|+\left\|x_{2 \varepsilon}^{\star}\right\|=1, \quad x_{1 \varepsilon}^{\star}+x_{2 \varepsilon}^{\star}=0 .
$$

This implies that $\left\|x_{1 \varepsilon}^{\star}\right\|=1 / 2>\varepsilon$ and the cone $\hat{N}(\cdot ; \Omega)$ is non-trivial at $x_{\varepsilon}$ which is a boundary point of $\Omega$ within $\varepsilon$ of $\bar{x}$. Therefore, the set (3.7) is norm dense in the boundary of $\Omega$.

Note that if $\Omega$ is convex, then the Fréchet normal cone $\hat{N}(\cdot ; \Omega)$ is reduced to the normal cone of convex analysis, and (3.7) coincides with the set of support points of $\Omega$ as in the Bishop-Phelps theorem [55, Theorem 3.18].

Now let us consider circumstances when one can pass to the limit in the results of Theorem 3.2 as $\varepsilon \downarrow 0$ and obtain in this way necessary conditions for extremal points of set systems $\left\{\Omega_{1}, \Omega_{2}\right\}$ in terms of the normal cone (2.2). We can always do it for any closed sets in finite dimensions and justify the extremal principle of the form

$$
\exists x^{\star} \neq 0 \text { such that } x^{\star} \in N\left(\bar{x} ; \Omega_{1}\right) \cap\left(-N\left(\bar{x} ; \Omega_{2}\right)\right) ;
$$

see Mordukhovich $[42,44,49]$ for alternative proofs and applications of this result in finite dimensional nonsmooth analysis and optimization. In infinite dimensions, one should impose additional assumptions on the sets in question to ensure the nontriviality of the sequential weak-star limit in the extremality conditions of Theorem 3.2. Some previous conditions in this direction can be found in $[32,34,44]$ for extremal systems in Fréchet differentiable spaces. In this paper we are able to establish (3.8) and related calculus results in the general Asplund space framework under the compactness condition as follows.

3.5. Definition. A closed set $\Omega \subset X$ is said to be normally compact around $\bar{x} \in \Omega$ if there exist positive numbers $\gamma, \delta$ and a compact subset $S$ of $X$ such that

$$
\hat{N}(x ; \Omega) \subset K_{\gamma}(S):=\left\{x^{\star} \in X^{\star}\left|\gamma\left\|x^{\star}\right\| \leq \max _{s \in S}\right|\left\langle x^{\star}, s\right\rangle \mid\right\} \quad \forall x \in B_{\delta}(\bar{x}) \cap \Omega .
$$

Condition (3.9) has been used by Loewen [38] to prove that the graph of the normal cone $(2.2)$ is closed in the norm $\times$ weak topology of $X \times X^{\star}$ for the case of reflexive spaces $X$. We observe that in the general Asplund space setting this condition does not ensure such a robustness property, but it turns out to be crucial to support limiting procedures developed below; see also [53] for more discussions.

In [38, Proposition 2.7], Loewen proves for the case of arbitrary Banach spaces that $\Omega$ satisfies the normal compactness condition (3.9) if it happens to be "compactly epi-Lipschitzian" around $\bar{x}$ in the sense of Borwein and Strojwas [7]. The 
latter means that there exist a neighborhood $U$ of $\bar{x}$, a neighborhood $V$ of the origin in $X$, a compact set $S \subset X$, and a constant $r>0$ such that

$$
\Omega \cap U+\lambda V \subset \Omega+\lambda S \quad \forall \lambda \in(0, r) .
$$

In particular, (3.9) holds for any set $\Omega$ epi-Lipschitzian around $\bar{x}$ in the sense of Rockafellar [58] that corresponds to (3.10) when $S$ is a singleton. Note that the epi-Lipschitzian property does not necessarily hold for arbitrary closed sets in finite dimensions. In fact, it is equivalent to the nonempty interiorty of Clarke's tangent cone to $\Omega$ at $\bar{x}$ that is reduced to int $\Omega \neq \emptyset$ if $\Omega$ is convex; see $[7,58]$. On the contrary, the compactly epi-Lipschitzian property is fulfilled if $\Omega$ is "epi-Lipschitzlike" around $\bar{x}$ in the sense of Borwein [3]. The latter always holds if either $\Omega$ is epi-Lipschitzian around $\bar{x}$ or $X$ is finite dimensional; see [3] for more details. Thus the normal compactness property in Definition 3.5 turns out to be a natural generalization of the epi-Lipschitzian behavior in Banach spaces and automatically holds for any closed set in finite dimensions.

3.6. Theorem. Let $X$ be an Asplund space, let $\Omega_{1}$ and $\Omega_{2}$ be closed subsets of $X$, and let $\bar{x} \in \Omega_{1} \cap \Omega_{2}$ be a locally extremal point of the system $\left\{\Omega_{1}, \Omega_{2}\right\}$. If one of the sets $\Omega_{1}$ and $\Omega_{2}$ is normally compact around $\bar{x}$, then there exists $x^{\star} \in X^{\star}$ such that the extremal principle (3.8) holds.

Proof. Let us use the extremality conditions of Theorem 3.2. Picking an arbitrary sequence $\varepsilon_{k} \downarrow 0$ as $k \rightarrow \infty$, we find sequences $\left\{x_{k}\right\} \subset X$ and $\left\{x_{k}^{\star}\right\} \subset X^{\star}$ such that

$$
\begin{gathered}
x_{i k}^{\star} \in \hat{N}\left(x_{i k} ; \Omega_{i}\right), \quad\left\|x_{i k}-\bar{x}\right\| \leq \varepsilon_{k} \text { for } i=1,2 ; \\
1 / 2-\varepsilon_{k} \leq\left\|x_{i k}^{\star}\right\| \leq 1 / 2+\varepsilon_{k}, i=1,2 \text {, and }\left\|x_{1 k}^{\star}+x_{2 k}^{\star}\right\| \leq \varepsilon_{k} .
\end{gathered}
$$

Using boundedness of $\left\{x_{i k}^{\star}\right\}$ and taking into account that the unit ball $B^{\star} \subset X^{\star}$ is weak-star sequentially compact for any Asplund space $X$, we may assume that $x_{i k}^{\star} \stackrel{w^{\star}}{\rightarrow} x_{i}^{\star}$ as $k \rightarrow \infty$ for $i=1,2$. Thus $x_{i}^{\star} \in N\left(\bar{x} ; \Omega_{i}\right), i=1,2$, and $x_{1}^{\star}+x_{2}^{\star}=0$ due to $(3.12)$.

Let us denote $x^{\star}:=x_{1}^{\star}=-x_{2}^{\star}$. To prove (3.8) it remains to show that $x^{\star} \neq 0$ if one of the sets $\Omega_{i}$ (say $\Omega_{1}$ ) is normally compact around $\bar{x}$. Assume that it is not true, i.e., $x_{1 k}^{\star} \stackrel{w^{\star}}{\rightarrow} x^{\star}=0$ as $k \rightarrow \infty$. Using the compactness of the set $S \subset X$ in (3.9), we conclude that $\left\langle x_{1 k}^{\star}, s\right\rangle \rightarrow 0$ uniformly in $s \in S$. By virtue of condition (3.9), this implies $x_{1 k}^{\star} \rightarrow 0$ as $k \rightarrow \infty$ in the norm topology of $X^{\star}$, which contradicts the first formula in (3.12) and completes the proof of the theorem.

3.7. Corollary. Let $X$ be an Asplund space and let $\Omega \subset X$ be normally compact around $\bar{x} \in \Omega$. Then $\bar{x}$ is a boundary point of $\Omega$ if and only if the normal cone $(2.2)$ is nontrivial, i.e.

$$
N(\bar{x} ; \Omega) \neq\{0\} .
$$

Proof. If $\bar{x}$ is an interior point of $\Omega$, then $N(\bar{x} ; \Omega)=\{0\}$ by virtue of Definition 2.1. Let $\bar{x}$ be a boundary point of $\Omega$, i.e., it is a locally extremal point of the system $\{\bar{x}, \Omega\}$. Using Theorem 3.6, we arrive at condition (3.13).

3.8. Remarks. (a) If both $\Omega_{1}$ and $\Omega_{2}$ are convex, then (3.8) is equivalent to the classical separation property:

$$
\exists x^{\star} \neq 0 \text { with }\left\langle x^{\star}, \omega_{1}\right\rangle \leq\left\langle x^{\star}, \omega_{2}\right\rangle \quad \forall \omega_{1} \in \Omega_{1}, \omega_{2} \in \Omega_{2} .
$$


It is easy to show that the separation property implies the local extremality of any point $\bar{x} \in \Omega_{1} \cap \Omega_{2}$ in the general nonconvex case; cf. [44, Proposition 6.1]. This means that for convex sets the separation and extremality properties are equivalent. In particular, any closed convex sets $\Omega_{1}$ and $\Omega_{2}$ with $\Omega_{1} \cap \Omega_{2} \neq \emptyset$ and (int $\Omega_{1}$ ) $\cap \Omega_{2}=$ $\emptyset$ provide an extremal system. Therefore, one may view Theorem 3.6 as a proper generalization of the classical separation (Hahn-Banach) theorem for nonconvex sets in Asplund spaces. Respectively, Corollary 3.7 turns out to be a nonconvex analogue of the supporting hyperplane result.

(b) As it has been mentioned above, the epi-Lipschitzian property of a convex set is reduced to the nonemptiness of its interiority. Thus the normal compactness condition (3.9) is a substantial development of this property in the general nonconvex setting. From this viewpoint the results of Theorem 3.2 may be treated as nonconvex generalizations of the classical separation theorem with no interiority-like assumptions.

(c) The results of Theorems 3.2 and 3.6 can be extended to an extremal system of sets $\left\{\Omega_{1}, \ldots, \Omega_{n}\right\}$ for any finite number $n \geq 2$, taking into account that Asplund spaces are invariant with respect to Cartesian product. This can be done either directly or using an inductive reduction to the case of two sets; cf. [32, 44, 51].

In conclusion of this section let us present a useful consequence of Corollary 3.7 that ensures nonemptiness of the subdifferential (2.7) for any locally Lipschitzian function defined on an Asplund space.

3.9. Corollary. Let $X$ be an Asplund space and let $\varphi: X \rightarrow \overline{\mathbf{R}}$ be Lipschitz continuous around $\bar{x}$. Then $\partial \varphi(\bar{x}) \neq \emptyset$.

Proof. Consider the set $\Omega:=$ epi $\varphi$, which is closed and epi-Lipschitzian around $(\bar{x}, \varphi(\bar{x}))$ due to the Lipschitz continuity of $\varphi$ around $\bar{x}$. Obviously, $(\bar{x}, \varphi(\bar{x}))$ is a boundary point of epi $\varphi$. Employing Corollary 3.7, one has $N((\bar{x}, \varphi(\bar{x}))$; epi $\varphi) \neq$ $\{0\}$. Now using Proposition 2.5, we conclude that the subdifferential (2.7) is nonempty.

3.10. Remark. One can easily see that the set $\partial \varphi(\bar{x})$ is bounded in the norm topology of $X^{\star}$ if $\varphi$ is Lipschitz continuous around $\bar{x}$ in any Banach space $X$. However, this set may not be weak-star closed even for Lipschitz functions defined on spaces with Fréchet differentiable renorms; see Section 9 for more details.

\section{SUM RULES FOR SUBDIFFERENTIALS}

In this section we obtain fundamental calculus results for the nonconvex sequential constructions in Section 2 related to representations of the subdifferentials for sums of extended-real-valued functions and the normal cone to intersections of sets in Asplund spaces. The main tool in proving these results is the extremal principle developed in the previous section.

First we consider normal compactness assumptions on functions related to the corresponding assumptions on sets in Section 3. We say that a l.s.c. function $\varphi: X \rightarrow \overline{\mathbf{R}}$ is normally compact around $\bar{x} \in \operatorname{dom} \varphi$ if its epigraph $\Omega:=$ epi $\varphi$ is normally compact around $(\bar{x}, \varphi(\bar{x}))$ in the sense of Definition 3.5.

It follows from Loewen [38] that $\varphi$ is normally compact around $\bar{x}$ if it is "compactly epi-Lipschitz" around this point. This corresponds to the compactly epiLipschitzian property of the set $\Omega:=$ epi $\varphi$ around $(\bar{x}, \varphi(\bar{x}))$; see Borwein [3]. A special case of this setting when the epigraph of $\varphi$ is epi-Lipschitzian around 
$(\bar{x}, \varphi(\bar{x}))$ corresponds to the directionally Lipschitz property of $\varphi$ around $\bar{x}$ in the sense of Rockafellar [58]. The latter always holds when $\varphi$ happens to be Lipschitz continuous around $\bar{x}$. Moreover, the class of compactly epi-Lipschitz functions covers "epi-Lipschitz-like" functions on Banach spaces that, in turn, include all l.s.c. functions defined on finite dimensional spaces; see [3]. Therefore, the normal compactness property under consideration holds for every l.s.c. function in finite dimensions as well as for locally Lipschitz functions and their generalizations in arbitrary Banach spaces.

The next theorem contains general sum rules for the sequential subdifferential constructions (2.7) and (2.8).

4.1. Theorem. Let $X$ be an Asplund space, let $\varphi_{i}: X \rightarrow \overline{\mathbf{R}}, i=1,2, \ldots, n$, be l.s.c. around $\bar{x}$, and let all but one of these functions be normally compact around $\bar{x}$. Suppose also that the following qualification condition holds:

(4.1) $\left[x_{i}^{\star} \in \partial^{\infty} \varphi_{i}(\bar{x}), i=1, \ldots, n \mid x_{1}^{\star}+\cdots+x_{n}^{\star}=0\right] \Longrightarrow x_{1}^{\star}=\cdots=x_{n}^{\star}=0$.

Then one has the inclusions

$$
\begin{gathered}
\partial\left(\varphi_{1}+\cdots+\varphi_{n}\right)(\bar{x}) \subset \partial \varphi_{1}(\bar{x})+\cdots+\partial \varphi_{n}(\bar{x}), \\
\partial^{\infty}\left(\varphi_{1}+\cdots+\varphi_{n}\right)(\bar{x}) \subset \partial^{\infty} \varphi_{1}(\bar{x})+\cdots+\partial^{\infty} \varphi_{n}(\bar{x}) .
\end{gathered}
$$

Moreover, if all $\varphi_{i}$ are subdifferentially regular at $\bar{x}$, then the sum $\varphi_{1}+\cdots+\varphi_{n}$ is also subdifferentially regular at this point, and equality holds in (4.2).

Proof. First let us consider the case of two functions $\varphi_{1}, \varphi_{2}$ and prove inclusion (4.2). For definiteness we assume that $\varphi_{1}$ is normally compact around $\bar{x}$.

Let $x^{\star} \in \partial\left(\varphi_{1}+\varphi_{2}\right)(\bar{x})$. Due to representation (2.16) one can find sequences $x_{k} \rightarrow \bar{x}$ and $x_{k}^{\star} \stackrel{w^{\star}}{\rightarrow} \bar{x}^{\star}$ such that $\varphi_{i}\left(x_{k}\right) \rightarrow \varphi_{i}(\bar{x}), i=1,2$, and $x_{k}^{\star} \in \hat{\partial}\left(\varphi_{1}+\varphi_{2}\right)\left(x_{k}\right)$, $k=1,2, \ldots$. Picking an arbitrary sequence $\varepsilon_{k} \downarrow 0$ as $k \rightarrow \infty$ and using (2.11) at $x_{k}$ for $\varepsilon=0$, we find a neighborhood $U_{k}$ of $x_{k}$ where

$$
\left(\varphi_{1}+\varphi_{2}\right)(x)-\left(\varphi_{1}+\varphi_{2}\right)\left(x_{k}\right)-\left\langle x_{k}^{\star}, x-x_{k}\right\rangle+\varepsilon_{k}\left\|x-x_{k}\right\| \geq 0 \forall x \in U_{k} .
$$

Without loss of generality, we assume that $\varphi_{1}$ and $\varphi_{2}$ are l.s.c. on $X$. Therefore, the sets

$$
\Omega_{1 k}:=\left\{(x, \mu) \in X \times \mathbf{R} \mid \varphi_{1}(x)-\varphi_{1}\left(x_{k}\right) \leq \mu\right\}
$$

and

$$
\Omega_{2 k}:=\left\{(x, \mu) \in X \times \mathbf{R} \mid \varphi_{2}(x)-\varphi_{2}\left(x_{k}\right)-\left\langle x_{k}^{\star}, x-x_{k}\right\rangle+\varepsilon_{k}\left\|x-x_{k}\right\| \leq-\mu\right\}
$$

are closed. By virtue of (4.4) and the construction of sets (4.5) and (4.6), we observe that $\left(x_{k}, 0\right)$ is a locally extremal point of the system $\left\{\Omega_{1 k}, \Omega_{2 k}\right\}$ for each $k=1,2, \ldots$ Indeed, one can easily check that

$\left(x_{k}, 0\right) \in \Omega_{1 k} \cap \Omega_{2 k}$ and $\Omega_{1 k} \cap\left[\Omega_{2 k}-(0, \nu)\right] \cap\left[U_{k} \times \mathbf{R}\right]=\emptyset \forall \nu>0, k=1,2, \ldots$.

Now we employ the extremal principle in Theorem 3.2. According to assertion (ii) of this theorem there exist $\left(x_{i k}, \mu_{i k}\right) \in\left(\right.$ epi $\left.\varphi_{i}\right) \cap B_{\varepsilon_{k}}\left(x_{k}, \varphi_{i}\left(x_{k}\right)\right), i=1,2$, as well as $\left(\tilde{x}_{k}^{\star}, \alpha_{k}\right) \in X^{\star} \times \mathbf{R}$ and $\left(\tilde{y}_{k}^{\star}, \beta_{k}\right) \in X^{\star} \times \mathbf{R}$ such that

(4.7) $1 / 2-\varepsilon_{k} \leq\left\|\tilde{x}_{k}^{\star}\right\|+\left|\alpha_{k}\right| \leq 1 / 2+\varepsilon_{k}, \quad 1 / 2-\varepsilon_{k} \leq\left\|\tilde{y}_{k}^{\star}\right\|+\left|\beta_{k}\right| \leq 1 / 2+\varepsilon_{k}$,

$$
\left(\tilde{x}_{k}^{\star}, \alpha_{k}\right) \in \hat{N}\left(\left(x_{1 k}, \mu_{1 k}-\varphi_{1}\left(x_{k}\right)\right) ; \Omega_{1 k}\right)
$$




$$
\begin{gathered}
\left(\tilde{y}_{k}^{\star}, \beta_{k}\right) \in \hat{N}\left(\left(x_{2 k},-\mu_{2 k}+\varphi_{2}\left(x_{k}\right)+\left\langle x_{k}^{\star}, x_{2 k}-x_{k}\right\rangle-\varepsilon_{k}\left\|x_{2 k}-x_{k}\right\|\right) ; \Omega_{2 k}\right), \\
\left\|\left(\tilde{x}_{k}^{\star}, \alpha_{k}\right)+\left(\tilde{y}_{k}^{\star}, \beta_{k}\right)\right\| \leq \varepsilon_{k} .
\end{gathered}
$$

Thus one has $\left(x_{i k}, \mu_{i k}\right) \stackrel{e p i}{\longrightarrow}\left(\bar{x}, \varphi_{i}(\bar{x})\right)$ as $k \rightarrow \infty$ for $i=1,2$. Taking into account that the space $X$ is Asplund and the sequences $\left.\left(\tilde{x}_{k}^{\star}, \alpha_{k}\right)\right\}$ and $\left\{\left(\tilde{y}_{k}^{\star}, \beta_{k}\right)\right\}$ are bounded, we may suppose that

$$
\left(\tilde{x}_{k}^{\star}, \alpha_{k}\right) \stackrel{w^{\star}}{\rightarrow}\left(\tilde{x}^{\star}, \alpha\right) \text { and }\left(\tilde{y}_{k}^{\star}, \beta_{k}\right) \stackrel{w^{\star}}{\rightarrow}\left(\tilde{y}^{\star}, \beta\right) \text { as } k \rightarrow \infty .
$$

From (4.5), (4.8), (4.11), and the construction of the normal cone (2.2) we get

$$
\left(\tilde{x}^{\star}, \alpha\right) \in N\left(\left(\bar{x}, \varphi_{1}(\bar{x})\right) ; \text { epi } \varphi_{1}\right) \text { with } \alpha \leq 0 .
$$

On the other hand, using (4.6), (4.9) as well as the definition of Fréchet normals, one obtains

$$
\limsup _{(x, \mu) \stackrel{e p_{i} \varphi_{2}}{\rightarrow}\left(x_{2 k}, \mu_{2 k}\right)} \frac{\left\langle\tilde{y}_{k}^{\star}, x-x_{2 k}\right\rangle-\beta_{k}\left(\mu-\mu_{2 k}-\left\langle x_{k}^{\star}, x-x_{2 k}\right\rangle+\varepsilon_{k}\left\|x-x_{2 k}\right\|\right)}{\|\left(x-x_{2 k}\left\|+\left|\mu-\mu_{2 k}\right|+\left|\left\langle x_{k}^{\star}, x-x_{2 k}\right\rangle\right|+\varepsilon_{k}\right\| x-x_{2 k} \|\right.} \leq 0 .
$$

The latter implies that

$$
\left(\beta_{k} x_{k}^{\star}+\tilde{y}_{k}^{\star},-\beta_{k}\right) \in \hat{N}_{\tilde{\varepsilon}_{k}}\left(\left(x_{2 k}, \mu_{2 k}\right) ; \operatorname{epi} \varphi_{2}\right)
$$

with $\tilde{\varepsilon}_{k}:=\varepsilon_{k}\left(1+\left\|x_{k}^{\star}\right\|+\varepsilon_{k}+\left|\beta_{k}\right|\right)$ for each $k=1,2 \ldots$ Passing to the limit in (4.13) as $k \rightarrow \infty$, one has $\left(\beta x^{\star}+\tilde{y}^{\star},-\beta\right) \in N\left(\left(\bar{x}, \varphi_{2}(\bar{x})\right)\right.$; epi $\left.\varphi_{2}\right)$, where $\tilde{y}^{\star}=-\tilde{x}^{\star}$ and $\beta=-\alpha$ by virtue of (4.10). Therefore, we obtain the inclusion

$$
\left(-\alpha x^{\star}-\tilde{x}^{\star}, \alpha\right) \in N\left(\left(\bar{x}, \varphi_{2}(\bar{x})\right) ; \operatorname{epi} \varphi_{2}\right) .
$$

Next let us show that $\alpha \neq 0$. Indeed, if $\alpha=0$, then (4.12) and (4.14) yield

$$
\left(\tilde{x}^{\star}, 0\right) \in N\left(\left(\bar{x}, \varphi_{1}(\bar{x})\right) ; \operatorname{epi} \varphi_{1}\right) \text { and }\left(-\tilde{x}^{\star}, 0\right) \in N\left(\left(\bar{x}, \varphi_{2}(\bar{x})\right) ; \operatorname{epi} \varphi_{2}\right),
$$

that implies $\tilde{x}^{\star}=0$ by virtue of definition (2.8) and the qualification condition (4.1) for $n=2$. Hence, $\left(\tilde{x}_{k}^{\star}, \alpha_{k}\right) \stackrel{w^{\star}}{\rightarrow}(0,0)$ as $k \rightarrow \infty$ due to (4.11). Now remembering that $\left(\tilde{x}_{k}^{\star}, \alpha_{k}\right) \in \hat{N}\left(\left(x_{1 k}, \mu_{1 k}\right)\right.$; epi $\left.\varphi_{1}\right)$ and $\varphi_{1}$ is normally compact around $\bar{x}$, we can conclude that $\left(\tilde{x}_{k}^{\star}, \alpha_{k}\right) \rightarrow(0,0)$ in the norm topology of $X^{\star} \times \mathbf{R}$ as $k \rightarrow \infty$; cf. the proof of Theorem 3.6. But the latter contradicts (4.7). Therefore, $\alpha \neq 0$ and inclusions (4.12) and (4.14) are reduced to, respectively,

$$
\begin{gathered}
\left(\tilde{x}^{\star} /|\alpha|,-1\right) \in N\left(\left(\bar{x}, \varphi_{1}(\bar{x})\right) ; \operatorname{epi} \varphi_{1}\right), \\
\left(x^{\star}-\tilde{x}^{\star} /|\alpha|,-1\right) \in N\left(\left(\bar{x}, \varphi_{2}(\bar{x})\right) ; \operatorname{epi} \varphi_{2}\right) .
\end{gathered}
$$

Denoting $x_{1}^{\star}:=\tilde{x}^{\star} /|\alpha|, x_{2}^{\star}:=x^{\star}-x_{1}^{\star}$ and using (2.7), we get from (4.15) the inclusion $x^{\star} \in \partial \varphi_{1}(\bar{x})+\partial \varphi_{2}(\bar{x})$, which proves (4.2) for the case of two functions.

To prove inclusion (4.3) for singular subdifferentials when $n=2$, we use the arguments above and Theorem 2.9(iii). When $n>2$, we prove inclusions (4.2) and (4.3) by induction, where the qualification assumption (4.1) at the current step is justified by using (4.3) at the previous step.

It remains to prove the equality and regularity statement of the theorem. Let us observe that one always has

$$
\hat{\partial}\left(\varphi_{1}+\cdots+\varphi_{n}\right)(\bar{x}) \supset \hat{\partial} \varphi_{1}(\bar{x})+\cdots+\hat{\partial} \varphi_{n}(\bar{x})
$$

for Fréchet subdifferentials of any functions $\varphi_{i}$. This easily follows from representation (2.11) as $\varepsilon=0$. If all $\varphi_{i}$ are subdifferentially regular at $\bar{x}$, then (4.2) and 
(4.16) ensure that the sum $\varphi_{1}+\cdots+\varphi_{n}$ is also subdifferentially regular at this point and equality holds in (4.2). This ends the proof of the theorem.

4.2. Remark. If all but one of the functions $\varphi_{i}$ are continuous around $\bar{x}$, then the conclusions of Theorem 4.1 hold true even when the remaining function (say $\varphi_{n}$ ) is not l.s.c. around $\bar{x}$. This follows from the definitions and the proof given above, where $\varphi_{n}$ can be replaced by its lower semicontinuous envelope; cf. the proof of Theorem 4.1 in [44].

4.3. Corollary. Let $X$ be an Asplund space and let all but one of the functions $\varphi_{i}$ be Lipschitz continuous around $\bar{x}$. Then:

(i) One has inclusion (4.2) where equality holds if all but one of the $\varphi_{i}$ are strictly differentiable at $\bar{x}$.

(ii) One always has equality in (4.3).

Proof. Due to Proposition 2.5 we have $\partial^{\infty} \varphi_{i}(\bar{x})=\{0\}$ if $\varphi_{i}$ is Lipschitz continuous around $\bar{x}$. This implies the qualification condition (4.1) and thus inclusion (4.2), taking into account Remark 4.2. To prove equality in (4.2) we consider for simplicity the case of two functions where $\varphi_{2}$ is strictly differentiable at $\bar{x}$. Then employing inclusion (4.2) for the sum $\varphi_{1}=\left(\varphi_{1}+\varphi_{2}\right)+\left(-\varphi_{2}\right)$ with $\partial\left(-\varphi_{2}\right)(\bar{x})=\left\{-\varphi_{2}^{\prime}(\bar{x})\right\}$, we obtain the inclusion opposite to (4.2). To prove equality in (4.3) in the Lipschitz case we use the same procedure, taking into account Proposition 2.5.

4.4. Remarks. (a) The equalities in Corollary 4.3 can be proved in any Banach space without using Theorem 4.1. This is directly based on the definitions and representation (2.12) with $\varepsilon>0$. We refer the reader to [52] for details and further considerations.

(b) It is well known that the condition $\partial^{\infty} \varphi(\bar{x})=\{0\}$, which is crucial in Corollary 4.3 , is not only sufficient but also necessary for the local Lipschitz continuity of l.s.c. functions defined on finite dimensional spaces; cf. [11, 44, 59, 63]. This result is no longer true in infinite dimensions unless additional conditions are imposed. A proper setting for the fulfilment of such a criterion of Lipschitz continuity is the normal compactness condition considered in Theorem 4.1, that covers the finite dimensional case. It can be derived from Theorem 8.8 stated below; cf. Loewen [40] for the case of spaces with Fréchet differentiable norms. An alternative proof of this criterion in Asplund spaces can be found in [53].

One can formulate other consequences of Theorem 4.1 that ensure the fulfilment of the normal compactness condition for all but one of the functions $\varphi_{i}$ (in particular, under directionally Lipschitz, Lipschitz-like, or compactly Lipschitz properties; see the discussions above). Let us present an important corollary for the normal cones to intersections of sets that is beyond the scope of locally Lipschitzian functions in Corollary 4.3.

4.5. Corollary. Let $X$ be an Asplund space and let $\Omega_{1}, \ldots, \Omega_{n}$ be closed sets in $X$ such that all but one of them are normally compact around $\bar{x} \in \Omega_{1} \cap \ldots \cap \Omega_{n}$. Suppose that

$$
\left[x_{i}^{\star} \in N\left(\bar{x} ; \Omega_{i}\right), i=1, \ldots, n \mid x_{1}^{\star}+\cdots+x_{n}^{\star}=0\right] \Longrightarrow x_{1}^{\star}=\cdots=x_{n}^{\star}=0 .
$$

Than one has the inclusion

$$
N\left(\bar{x} ; \Omega_{1} \cap \ldots \cap \Omega_{n}\right) \subset N\left(\bar{x} ; \Omega_{1}\right)+\cdots+N\left(\bar{x} ; \Omega_{n}\right)
$$

where equality holds when all $\Omega_{i}$ are regular at $\bar{x}$. 
Proof. Taking $\varphi_{i}:=\delta\left(\cdot, \Omega_{i}\right)$ in Theorem 4.1 and using representations (2.14), we reduce the qualification condition (4.1) to (4.17) and both inclusions (4.2) and (4.3) to (4.18). To finish the proof we observe that the subdifferential regularity of $\delta(\cdot, \Omega)$ is equivalent to the regularity of $\Omega$ at the same point.

4.6. Remark. In finite dimensions the results of Theorem 4.1 and Corollary 4.5 for extended-real-valued l.s.c. functions and closed sets were first obtained in Mordukhovich [43, 44]; we also refer the reader to [11, 22, 39, 45, 63] for various proofs, special cases, and discussions. In [31], Kruger proved the infinite dimensional counterparts of those results for directionally Lipschitzian functions and epi-Lipschitzian sets in spaces with Fréchet differentiable renorms under more restrictive qualification conditions. The results presented above unify and extend both finite and infinite dimensional predecessors to the Asplund space setting under the most general qualification and compactness conditions. Note that Ioffe [24] established analogues of the results in Theorem 4.1 for his $G$-subdifferentials of directionally Lipschitz functions in Banach spaces which are not reduced to the results proved above even for the case of locally Lipschitz functions defined on spaces with Fréchet differentiable renorms; see Section 9. Recently Jourani and Thibault [30] obtained generalizations of the latter results for $G$-subdifferentials to the case of compactly epi-Lipschitz functions.

\section{SCALARIZATION FORMUla}

In Section 2 we defined the coderivative (2.4) of a multifunction $\Phi: X \Rightarrow Y$ using the normal cone to its graph. This concept turns out to be important for many applications, some of which are considered in this paper; see the next section. Actually our basic subdifferential construction (2.7) is defined geometrically as a special case of the coderivative, although it admits equivalent analytical representations. In some situations (in particular, for chain rules; see below) it is more convenient to find reverse representations of the coderivatives for multi-valued or single-valued mappings in terms of the subdifferentials for related scalarizations. Such a representation was obtained for single-valued locally Lipschitzian mappings $\Phi: X \rightarrow Y$ between finite dimensional spaces in the form

$$
D^{\star} \Phi(\bar{x})\left(y^{\star}\right)=\partial\left\langle y^{\star}, \Phi\right\rangle(\bar{x}) \text { for all } y^{\star} \in Y^{\star}
$$

where $\left\langle y^{\star}, \Phi\right\rangle(x):=\left\langle y^{\star}, \Phi(x)\right\rangle$ is the Lagrange scalarization of $\Phi$; cf. the proofs in Ioffe [22, Proposition 8] and Mordukhovich [44, Theorem 3.3] with further discussions and references. The question arises about possible extensions of the scalarization formula (5.1) to infinite dimensional spaces. Now we provide such an extension for the following subclass of locally Lipschitzian mappings in infinite dimensions.

5.1. Definition. Let $\Phi: X \rightarrow Y$ be a single-valued mapping between Banach spaces $X$ and $Y$ that is Lipschitz continuous around $\bar{x}$. We say that $\Phi$ is strictly Lipschitzian at $\bar{x}$ if there exists a neighborhood $V$ of the origin in $X$ such that the sequence

$$
\frac{\Phi\left(x_{k}+t_{k} v\right)-\Phi\left(x_{k}\right)}{t_{k}}, \quad k=1,2, \ldots
$$

has a convergent subsequence in the norm topology of $Y$ for each $v \in V, x_{k} \rightarrow \bar{x}$, and $t_{k} \downarrow 0$ as $k \rightarrow \infty$. 
Obviously, any mapping $\Phi: X \rightarrow Y$ that is Lipschitz continuous around $\bar{x}$ is strictly Lipschitzian at this point if the space $Y$ is finite dimensional. Moreover, one can check directly from the definitions that any locally Lipschitzian mapping acting between Banach spaces is strictly Lipschitzian at $\bar{x}$ if it has a norm-compact-valued "strict prederivative" in the sense of Ioffe [20]. Thus the class of strictly Lipschitzian mappings covers all strictly differentiable mappings and all compositions $H \circ F$ of a Lipschitz continuous mapping $F$ with a strictly differentiable mapping $H$ whose derivative is a compact operator. The latter subclass includes Fredholm integral operators with Lipschitz continuous kernels, which are particularly important for applications in optimal control; see [19].

Also one can easily verify that $\Phi$ is strictly Lipschitzian at $\bar{x}$ in the general Banach space setting if it is "strongly compactly Lipschitzian" at this point in the sense intensively studied by Jourani and Thibault; see, e.g., [30]. After receiving this paper, Thibault proved (personal communication) the equivalence of our Definition 5.1 to a variant [29] of his original concept of "compactly Lipschitzian" mappings; cf. [64].

5.2. Theorem. Let $X$ and $Y$ be Banach spaces and let $\Phi: X \rightarrow Y$ be a singlevalued mapping continuous around $\bar{x}$. Then one always has

$$
\partial\left\langle y^{\star}, \Phi\right\rangle(\bar{x}) \subset D^{\star} \Phi(\bar{x})\left(y^{\star}\right) \forall y^{\star} \in Y^{\star} .
$$

If, in addition, $X$ is an Asplund space and $\Phi$ is strictly Lipschitzian at $\bar{x}$, then one has the scalarization formula $(5.1)$, where $D^{\star} \Phi(\bar{x})\left(y^{\star}\right) \neq \emptyset$ for any $y^{\star} \in Y^{\star}$.

Proof. First let us prove inclusion (5.2) under the general assumptions. Taking any $x^{\star} \in \partial\left\langle y^{\star}, \Phi\right\rangle(\bar{x})$ and using representation (2.12), we find sequences $\left\{x_{k}\right\},\left\{x_{k}^{\star}\right\}$, and $\left\{\varepsilon_{k}\right\}$ such that $x_{k} \rightarrow \bar{x}, x_{k}^{\star} \stackrel{w^{\star}}{\rightarrow} x^{\star}$, and $\varepsilon_{k} \downarrow 0$ as $k \rightarrow \infty$ with $x_{k}^{\star} \in \tilde{\partial}_{\varepsilon_{k}}\left\langle y^{\star}, \Phi\right\rangle\left(x_{k}\right)$ for $k=1,2, \ldots$ Due to (2.11) there is a sequence $\delta_{k} \downarrow 0$ as $k \rightarrow \infty$ with

$$
\begin{array}{r}
\left\langle y^{\star}, \Phi\right\rangle(x)-\left\langle y^{\star}, \Phi\right\rangle\left(x_{k}\right)-\left\langle x_{k}^{\star}, x-x_{k}\right\rangle \geq-2 \varepsilon_{k}\left\|x-x_{k}\right\| \\
\forall x \in B_{\delta_{k}}\left(x_{k}\right), k=1,2, \ldots .
\end{array}
$$

The latter easily implies

$$
\limsup _{x \rightarrow x_{k}} \frac{\left\langle x_{k}^{\star}, x-x_{k}\right\rangle-\left\langle y^{\star}, \Phi(x)-\Phi\left(x_{k}\right)\right\rangle}{\left\|\left(x-x_{k}, \Phi(x)-\Phi\left(x_{k}\right)\right)\right\|} \leq 2 \varepsilon_{k},
$$

that means $\left(x_{k}^{\star},-y^{\star}\right\rangle \in \hat{N}_{2 \varepsilon_{k}}\left(\left(x_{k}, \Phi\left(x_{k}\right)\right)\right.$; gph $\left.\Phi\right)$ for each $k=1,2, \ldots$ Now using (2.2) and (2.4), we conclude that $x^{\star} \in D^{\star} \Phi(\bar{x})\left(y^{\star}\right)$. This proves inclusion (5.2) in the general case under consideration.

Now let us prove that the opposite inclusion holds if $X$ is an Asplund space and $\Phi$ is strictly Lipschitzian at $\bar{x}$. Picking any $x^{\star} \in D^{\star} \Phi(\bar{x})\left(y^{\star}\right)$ and using definitions (2.4) and (2.2), we find sequences $x_{k}^{\star} \stackrel{w^{\star}}{\rightarrow} x^{\star}, y_{k}^{\star} \stackrel{w^{\star}}{\rightarrow} y^{\star}, x_{k} \rightarrow \bar{x}$, and $\varepsilon_{k} \downarrow 0$ as $k \rightarrow \infty$ with $\left(x_{k}^{\star},-y_{k}^{\star}\right) \in \hat{N}_{\varepsilon_{k}}\left(\left(x_{k}, \Phi\left(x_{k}\right)\right)\right.$; gph $\left.\Phi\right)$ for $k=1,2, \ldots$ By virtue of (2.1) there exists a sequence $\delta_{k} \downarrow 0$ as $k \rightarrow \infty$ such that

$$
\left\langle x_{k}^{\star}, x-x_{k}\right\rangle-\left\langle y_{k}^{\star}, \Phi(x)-\Phi\left(x_{k}\right)\right\rangle \leq 2 \varepsilon_{k}\left(\left\|x-x_{k}\right\|+\left\|\Phi(x)-\Phi\left(x_{k}\right)\right\|\right)
$$

for any $x \in B_{\delta_{k}}\left(x_{k}\right)$ with $\left\|\Phi(x)-\Phi\left(x_{k}\right)\right\| \leq \delta_{k}, k=1,2, \ldots$ Taking into account that $\Phi$ is locally Lipschitzian around $\bar{x}$ and $x_{k} \rightarrow \bar{x}$ as $k \rightarrow \infty$, we consider a uniform Lipschitz modulus $l$ for $\Phi$ on the sets $B_{\delta_{k}}\left(x_{k}\right)$ for all $k=1,2, \ldots$. Then 
(5.3) provides

$$
\begin{aligned}
&\left\langle x_{k}^{\star}, x-x_{k}\right\rangle-\left\langle y_{k}^{\star}, \Phi(x)\right\rangle+\left\langle y_{k}^{\star}, \Phi\left(x_{k}\right)\right\rangle \leq 2 \varepsilon_{k}(1+l)\left\|x-x_{k}\right\| \\
& \forall x \in B_{\delta_{k}}\left(x_{k}\right), k=1,2, \ldots .
\end{aligned}
$$

The latter implies that for each fixed $k=1,2, \ldots$ one has the limiting expression

$$
\liminf _{x \rightarrow x_{k}} \frac{\left\langle y_{k}^{\star}, \Phi\right\rangle(x)-\left\langle y_{k}^{\star}, \Phi\right\rangle\left(x_{k}\right)-\left\langle x_{k}^{\star}, x-x_{k}\right\rangle}{\left\|x-x_{k}\right\|} \geq \nu_{k},
$$

where $\nu_{k}:=2(1+l) \varepsilon_{k} \rightarrow 0$ as $k \rightarrow \infty$. Remembering (2.11), we obtain

$$
x_{k}^{\star} \in \tilde{\partial}_{\nu_{k}}\left\langle y_{k}^{\star}, \Phi\right\rangle\left(x_{k}\right)=\tilde{\partial}_{\nu_{k}}\left[\left\langle y^{\star}, \Phi\right\rangle+\left\langle y_{k}^{\star}-y^{\star}, \Phi\right\rangle\right]\left(x_{k}\right) .
$$

Now we consider the sum of two functions in (5.4) and employ Proposition 2.7 with $\varepsilon=\delta=\gamma:=\nu_{k}$ for each $k=1,2, \ldots$ In this way one finds sequences $\left\{u_{k}\right\},\left\{v_{k}\right\},\left\{z_{k}^{\star}\right\}$, and $\left\{v_{k}^{\star}\right\}$ such that $\left\|u_{k}-x_{k}\right\| \leq \nu_{k},\left\|v_{k}-x_{k}\right\| \leq \nu_{k},\left\|z_{k}^{\star}\right\| \leq \nu_{k}$, and $x_{k}^{\star}=\tilde{x}_{k}^{\star}+z_{k}^{\star}$ with

$$
\tilde{x}_{k}^{\star}=u_{k}^{\star}+v_{k}^{\star}, u_{k}^{\star} \in \hat{\partial}\left\langle y^{\star}, \Phi\right\rangle\left(u_{k}\right) \text {, and } v_{k}^{\star} \in \hat{\partial}\left\langle y_{k}^{\star}-y^{\star}, \Phi\right\rangle\left(v_{k}\right) \text { for } k=1,2 \ldots
$$

Taking into account the boundedness of $\left\{y_{k}^{\star}\right\}$ in $Y^{\star}$ and the Lipschitz continuity of $\Phi$ around $\bar{x}$, we conclude that the sequences $\left\{u_{k}^{\star}\right\}$ and $\left\{v_{k}^{\star}\right\}$ are also bounded in $Y^{\star}$. Thus we can suppose that $u_{k}^{\star} \stackrel{w^{\star}}{\rightarrow} u^{\star}$ and $v_{k}^{\star} \stackrel{w^{\star}}{\rightarrow} v^{\star}$ as $k \rightarrow \infty$. Now passing to the limit in (5.5), one gets $u^{\star} \in \partial\left\langle y^{\star}, \Phi\right\rangle(\bar{x})$ and $x^{\star}=u^{\star}+v^{\star}$.

We claim that $v^{\star}=0$. Indeed, it follows from (5.5) and (2.11) as $\varepsilon=0$ that for any $\gamma_{k} \downarrow 0$ there exists $\delta_{k} \downarrow 0$ as $k \rightarrow \infty$ such that

$$
\left\langle v_{k}^{\star}, x-v_{k}\right\rangle-\left\langle y_{k}^{\star}-y^{\star}, \Phi(x)-\Phi\left(v_{k}\right)\right\rangle \leq \gamma_{k}\left\|x-v_{k}\right\|
$$

for all $x \in B_{\delta_{k}}\left(v_{k}\right), k=1,2, \ldots$ Let us consider a neighborhood $V$ of the origin in $X$ that appeared in Definition 5.1. Picking any $v \in V$, one can choose a sequence $t_{k} \downarrow 0$ as $k \rightarrow \infty$ such that $v_{k}+t_{k} v \in B_{\delta_{k}}\left(v_{k}\right)$ for all $k$. Then (5.6) implies

$$
\left\langle v_{k}^{\star}, v\right\rangle-\left\langle y_{k}^{\star}-y^{\star}, \frac{\Phi\left(v_{k}+t_{k} v\right)-\Phi\left(v_{k}\right)}{t_{k}}\right\rangle \leq \gamma_{k}\|v\| .
$$

Since $\Phi$ is strictly Lipschitzian at $\bar{x}$, we may assume that the sequence $\left\{\left[\Phi\left(v_{k}+t_{k} v\right)-\Phi\left(x_{k}\right)\right] / t_{k}\right\}$ converges in the norm topology of $Y$ as $k \rightarrow \infty$. Now taking the limit in (5.7) as $k \rightarrow \infty$, one has $\left\langle v^{\star}, v\right\rangle \leq 0$ for any $v \in V$. This obviously implies $v^{\star}=0$. Therefore, we get $x^{\star} \in \partial\left\langle y^{\star}, \Phi\right\rangle(\bar{x})$ and prove the opposite inclusion in (5.2), i.e., the scalarization formula (5.1). The nonemptiness of $D^{\star} \Phi(\bar{x})\left(y^{\star}\right)$ for any $y^{\star} \in Y^{\star}$ follows from (5.1) and Corollary 3.9. This ends the proof of the theorem.

5.3. Remark. In [24, Theorem 7.8], Ioffe established an analogue of Theorem 5.2 for the so-called " $G$-nuclei", that may be bigger than our sequential constructions even for locally Lipschitzian mappings $\Phi: X \rightarrow Y$ between spaces with Fréchet differentiable renorms; see Section 9. The corresponding $G$-analogue of the scalarization formula (5.1) was proved in [24] for the case of locally Lipschitzian mappings $\Phi$ that have strict prederivatives at $\bar{x}$ with norm compact values and, therefore, belong to the class of strictly Lipschitzian mappings in Definition 5.1. 


\section{Subdifferentials of MARginal FunCtions AND CHAin RULES}

In this section we study a broad class of marginal functions of the form

$$
m(x):=\inf \{\varphi(x, y) \mid y \in \Phi(x)\},
$$

where $\varphi: X \times Y \rightarrow \overline{\mathbf{R}}$ is an extended-real-valued function and $\Phi: X \Rightarrow Y$ is a multifunction between Banach spaces. It is well known that marginal functions play a substantial role in nonsmooth analysis and optimization. In particular, they include value functions in various optimization problems, that characterize the dependence of the optimal value in the problem on parametric perturbations. We observe that the marginal function (6.1) happens to be nonsmooth even in classical situations with a smooth function $\varphi$ and a simple constant multifunction $\Phi$. To study generalized differential properties of marginal functions is one of the principal topics in sensitivity analysis.

In what follows we obtain comprehensive results on evaluating the subdifferentials (2.7) and (2.8) for the class of marginal functions (6.1) in Asplund spaces. Note that if $\varphi(x, y)=\varphi(y)$ and $\Phi$ is single-valued, the marginal function (6.1) coincides with the composition

$$
(\varphi \circ \Phi)(x):=\varphi(\Phi(x)),
$$

and subdifferential formulas for (6.1) are reduced to chain rules in nonsmooth subdifferential calculus that are crucial to the theory and applications of nonsmooth analysis. In this way we obtain refined chain rules for the subdifferentials under consideration using the scalarization formula in Section 5.

The main results of this section consist of three theorems that are generally independent and different in proofs, although they have essential intersections. To formulate the results let us consider the parametric minimum set

$$
M(x):=\{y \in \Phi(x) \mid \varphi(x, y)=m(x)\}
$$

associated with (6.1). We always assume that the multifunction $M: X \Rightarrow Y$ in (6.3) has the following lower semicompactness property around the reference point $\bar{x}$ : there exists a neighborhood $U$ of $\bar{x}$ such that for any $x \in U$ and any sequence $x_{k} \rightarrow x$ as $k \rightarrow \infty$ there is a sequence $y_{k} \in M\left(x_{k}\right), k=1,2, \ldots$, which contains a subsequence convergent in the norm topology of $Y$. Obviously, any multifunction lower semicontinuous around $\bar{x}$ is lower semicompact around this point. If $\operatorname{dim} Y$ $<\infty$, the lower semicompactness property is inherent in any multifunction whose values are nonempty and uniformly bounded around $\bar{x}$.

Our first theorem on the subdifferentiation of (6.1) is concerned with general nonsmooth functions $\varphi$ and multifunctions $\Phi$ in Asplund spaces and generalizes to infinite dimensions the results and arguments in Mordukhovich [43, 44, 45]. In its formulation we use the normal compactness conditions for sets and functions discussed in Sections 3 and 4. The proof is related to employing the sum rule in Theorem 4.1, which is based on the extremal principle.

6.1. Theorem. Let $\Phi: X \Rightarrow Y$ be a closed-graph multifunction between Asplund spaces $X$ and $Y$, let $\varphi: X \times Y \rightarrow \overline{\mathbf{R}}$ be l.s.c. on gph $\Phi$, and let the multifunction $M$ in (6.3) be lower semicompact around $\bar{x} \in \operatorname{dom} m$. Assume that for any $\bar{y} \in M(\bar{x})$ either $\varphi$ or gph $\Phi$ is normally compact around $(\bar{x}, \bar{y})$ and the qualification condition

$$
\nexists\left(x^{\star}, y^{\star}\right) \in \partial^{\infty} \varphi(\bar{x}, \bar{y}) \text { with }\left(y^{\star},-x^{\star}\right) \in \operatorname{gph} D^{\star} \Phi(\bar{x}, \bar{y})
$$


holds. Then one has the inclusions

$$
\begin{gathered}
\partial m(\bar{x}) \subset \bigcup\left[x^{\star}+D^{\star} \Phi(\bar{x}, \bar{y})\left(y^{\star}\right) \mid\left(x^{\star}, y^{\star}\right) \in \partial \varphi(\bar{x}, \bar{y}), \bar{y} \in M(\bar{x})\right], \\
\left.\partial^{\infty} m(\bar{x}) \subset \bigcup\left[x^{\star}+D^{\star} \Phi(\bar{x}, \bar{y})\left(y^{\star}\right) \mid\left(x^{\star}, y^{\star}\right) \in \partial^{\infty} \varphi(\bar{x}, \bar{y})\right), \bar{y} \in M(\bar{x})\right] .
\end{gathered}
$$

Moreover, $m$ is subdifferentially regular at $\bar{x}$ and (6.5) becomes an equality if $\Phi$ is single-valued around $\bar{x}, \varphi$ is subdifferentially regular at $(\bar{x}, \Phi(\bar{x}))$ and either

(a) $\operatorname{dim} Y<\infty, \Phi$ is Lipschitz continuous around $\bar{x}$ with gph $\Phi$ regular at $(\bar{x}, \Phi(\bar{x}))$, or

(b) $\Phi$ is strictly differentiable at $\bar{x}$.

Proof. First observe that the marginal function (6.1) is l.s.c. around $\bar{x}$ under the general assumptions made. Indeed, let $U$ be a neighborhood of $\bar{x}$ from the lower semicompactness condition for $M$. Taking any $x \in U$ and sequence $x_{k} \rightarrow x$, we find a sequence $y_{k} \in M\left(x_{k}\right)$ that contains a subsequence convergent to some point $y \in Y$. Since $\Phi$ has closed graph, one gets $(x, y) \in$ gph $\Phi$. Now using the l.s.c. of $\varphi$ on gph $\Phi$, we obtain the relationships

$$
m(x) \leq \varphi(x, y) \leq \liminf _{k \rightarrow \infty} \varphi\left(x_{k}, y_{k}\right)=\liminf _{k \rightarrow \infty} m\left(x_{k}\right),
$$

that establish the l.s.c. of $m(x)$ around $\bar{x}$.

Let us consider the function $f: X \times Y \rightarrow \overline{\mathbf{R}}$ defined by

$$
f(x, y):=\varphi(x, y)+\delta((x, y), \operatorname{gph} \Phi),
$$

and let us prove that

$$
\partial m(\bar{x}) \subset\left\{x^{\star} \in X^{\star} \mid\left(x^{\star}, 0\right) \in \partial f(\bar{x}, \bar{y}), \bar{y} \in M(\bar{x})\right\} .
$$

Since $m(x)$ is l.s.c. around $\bar{x}$, we use Theorem 2.9(iii) and for any $x^{\star} \in \partial m(\bar{x})$ find sequences $x_{k} \rightarrow \bar{x}$ and $x_{k}^{\star} \stackrel{w^{\star}}{\rightarrow} x^{\star}$ such that $m\left(x_{k}\right) \rightarrow m(\bar{x})$ as $k \rightarrow \infty$ and $x_{k}^{\star} \in \hat{\partial} m\left(x_{k}\right)$ for all $k=1,2, \ldots$ By virtue of (2.11), for any $\varepsilon>0$ there exists a sequence $\eta_{k} \downarrow 0$ as $k \rightarrow \infty$ with

$$
\left\langle x_{k}^{\star}, x-x_{k}\right\rangle \leq m(x)-m\left(x_{k}\right)+\varepsilon\left\|x-x_{k}\right\| \forall x \in B_{\eta_{k}}\left(x_{k}\right), k=1,2, \ldots .
$$

Due to (6.7), this implies

$$
\left(x_{k}^{\star}, 0\right) \in \hat{\partial} f\left(x_{k}, y_{k}\right) \forall y_{k} \in M\left(x_{k}\right), k=1,2, \ldots
$$

Now using the lower semicompactness of $M$ at $\bar{x}$, one can select a sequence $y_{k} \in$ $M\left(x_{k}\right)$ that contains a subsequence convergent to some point $\bar{y} \in \Phi(\bar{x})$. Since $m\left(x_{k}\right) \rightarrow m(\bar{x})$, one concludes that $\bar{y} \in M(\bar{x})$ and $f\left(x_{k}, y_{k}\right) \rightarrow f(\bar{x}, \bar{y})$ as $k \rightarrow \infty$. Employing (6.9) and (2.16), we arrive at $\left(x^{\star}, 0\right) \in \partial f(\bar{x}, \bar{y})$ and finish the proof of (6.8).

Next we use Theorem 4.1 to represent the subdifferential $\partial f(\bar{x}, \bar{y})$ in $(6.8)$ for the sum of two functions (6.7). One can easily check that the qualification condition (4.1) is reduced to (6.4), and inclusions (4.2) and (6.8) imply (6.5).

To establish inclusion (6.6) for the singular subdifferentials we observe that

$$
\partial^{\infty} m(\bar{x}) \subset\left\{x^{\star} \in X^{\star} \mid\left(x^{\star}, 0\right) \in \partial^{\infty} f(\bar{x}, \bar{y}), \bar{y} \in M(\bar{x})\right\} .
$$

This can be proved similarly to (6.8) based on the last representation in (2.17). Then we derive (6.6) from (6.10) employing inclusion (4.3) in Theorem 4.1. 
It remains to consider the equality cases in the theorem when $\Phi$ is single-valued around $\bar{x}$ and $m(x)=\varphi(x, \Phi(x))$. First we are going to show that

$$
\partial m(\bar{x})=\left\{x^{\star} \in X^{\star} \mid\left(x^{\star}, 0\right) \in \partial f(\bar{x}, \Phi(\bar{x}))\right\}
$$

if either $\Phi$ is strictly differentiable at $\bar{x}$ or $\Phi$ is Lipschitz continuous around $\bar{x}$ and $Y$ is finite dimensional.

Indeed, let $\Phi: X \rightarrow Y$ be Lipschitz continuous around $\bar{x}$ with modulus $l \geq 0$ and let $\left(x^{\star}, 0\right) \in \partial f(\bar{x}, \Phi(\bar{x}))$. Using (2.16) and (6.7), one can find sequences $x_{k} \rightarrow$ $\bar{x}, x_{k}^{\star} \stackrel{w^{\star}}{\rightarrow} x^{\star}$, and $y_{k}^{\star} \stackrel{w^{\star}}{\rightarrow} 0$ such that $\varphi\left(x_{k}, \Phi\left(x_{k}\right)\right) \rightarrow \varphi(\bar{x}, \Phi(\bar{x}))$ as $k \rightarrow \infty$ and

$$
\liminf _{x \rightarrow x_{k}} \frac{\varphi(x, \Phi(x))-\varphi\left(x_{k}, \Phi\left(x_{k}\right)\right)-\left\langle\left(x_{k}^{\star}, y_{k}^{\star}\right),(x, \Phi(x))-\left(x_{k}, \Phi\left(x_{k}\right)\right)\right\rangle}{\left\|(x, \Phi(x))-\left(x_{k}, \Phi\left(x_{k}\right)\right)\right\|} \geq 0
$$

for each $k=1,2, \ldots$ If $\operatorname{dim} Y<\infty$, then $\left\|y_{k}^{\star}\right\| \rightarrow 0$, and (6.12) implies

$$
\liminf _{x \rightarrow x_{k}} \frac{\varphi(x, \Phi(x))-\varphi\left(x_{k}, \Phi\left(x_{k}\right)\right)-\left\langle x_{k}^{\star}, x-x_{k}\right\rangle}{\left\|x-x_{k}\right\|} \geq-\varepsilon_{k},
$$

where $\varepsilon_{k}:=(l+1)\left\|y_{k}^{\star}\right\| \rightarrow 0$ as $k \rightarrow \infty$. Therefore, $x_{k}^{\star} \in \tilde{\partial}_{\varepsilon_{k}} m\left(\bar{x}_{k}\right)$ for each $k$, and we get $x^{\star} \in \partial m(\bar{x})$ by virtue of (2.12). This proves equality (6.11) for the case of $\operatorname{dim} Y<\infty$.

Next we prove that if $\Phi$ is strictly differentiable at $\bar{x}$, equality (6.11) holds with no finite dimensionality assumption. It is well known that any mapping strictly differentiable at $\bar{x}$ is Lipschitz continuous around $\bar{x}$ with some modulus $l \geq 0$. By definition (2.6) of the strict derivative, for any sequence $\gamma_{\nu} \downarrow 0$ there is a sequence $\eta_{\nu} \downarrow 0$ as $\nu \rightarrow \infty$ such that

$$
\left\|\Phi(u)-\Phi(x)-\Phi^{\prime}(\bar{x})(u-x)\right\| \leq \gamma_{\nu}\|u-x\| \quad \forall x, u \in B_{\eta_{\nu}}(\bar{x}), \nu=1,2, \ldots .
$$

Using (6.12) and (6.13), we can select subsequences $k_{\nu} \rightarrow \infty$ and $x_{k_{\nu}} \rightarrow \bar{x}$ as $\nu \rightarrow \infty$ along which one has

$$
\begin{aligned}
& \varphi(x, \Phi(x))-\varphi\left(x_{k_{\nu}}, \Phi\left(x_{k_{\nu}}\right)\right)-\left\langle x_{k_{\nu}}^{\star}+\left(\Phi^{\prime}(\bar{x})\right)^{\star} y_{k_{\nu}}^{\star}, x-x_{k_{\nu}}\right\rangle \\
& \quad \leq-\gamma_{\nu}\left\|y_{k_{\nu}}^{\star}\right\|(1+l)\left\|x-x_{k \nu}\right\| \forall x \in B_{\eta_{\nu} / 2}\left(x_{k_{\nu}}\right), \nu=1,2, \ldots .
\end{aligned}
$$

The latter implies that

$$
x_{k_{\nu}}^{\star}+\left(\Phi^{\prime}(\bar{x})\right)^{\star} y_{k_{\nu}}^{\star} \in \tilde{\partial}_{\varepsilon_{\nu}} \varphi\left(x_{k_{\nu}}, \Phi\left(x_{k_{\nu}}\right)\right), \quad \nu=1,2, \ldots,
$$

where $\varepsilon_{\nu}:=\gamma_{\nu}\left\|y_{k_{\nu}}^{\star}\right\|(1+l) \rightarrow 0$ as $\nu \rightarrow \infty$. Passing to the limit in (6.14) and observing that $x_{k_{\nu}}^{\star}+\left(\Phi^{\prime}(\bar{x})\right)^{\star} y_{k_{\nu}}^{\star} \stackrel{w^{\star}}{\rightarrow} x^{\star}$ as $\nu \rightarrow \infty$, we obtain $x^{\star} \in \partial m(\bar{x})$ by virtue of (2.12). This proves equality (6.11) for the case of strictly differentiable mappings $\Phi$ between Asplund spaces.

To finish the proof of the theorem it remains to apply the subdifferential regularity statement in Theorem 4.1 to the sum of functions (6.7) in equality (6.11) under the assumptions made. Due to Proposition 2.3 the strict differentiability of $\Phi$ at $\bar{x}$ ensures the regularity of its graph at $(\bar{x}, \Phi(\bar{x}))$ for any Banach spaces. Note that the latter properties are equivalent for locally Lipschitzian mappings between finite dimensional spaces (cf. $[48,62]$ ), i.e., case (b) is contained in (a) if both $X$ and $Y$ are finite dimensional. 
6.2. Remark. One can easily see that for $\varphi(x, y)=\varphi(y)$ the qualification condition (6.4) is reduced to

$$
\partial^{\infty} \varphi(\bar{y}) \cap \operatorname{ker} D^{\star} \Phi(\bar{x}, \bar{y})=\{0\} \quad \forall \bar{y} \in M(\bar{x}),
$$

that is automatically fulfilled if, in particular, $\varphi$ is Lipschitz continuous around $\bar{y}$; cf. Proposition 2.5. Respectively, inclusions (6.5) and (6.6) can be written as

$$
\begin{gathered}
\partial m(\bar{x}) \subset \bigcup\left[D^{\star} \Phi(\bar{x}, \bar{y})\left(y^{\star}\right) \mid y^{\star} \in \partial \varphi(\bar{y}), \bar{y} \in M(\bar{x})\right], \\
\partial^{\infty} m(\bar{x}) \subset \bigcup\left[D^{\star} \Phi(\bar{x}, \bar{y})\left(y^{\star}\right) \mid y^{\star} \in \partial^{\infty} \varphi(\bar{y}), \bar{y} \in M(\bar{x})\right] .
\end{gathered}
$$

If $\Phi$ is single-valued and continuous around $\bar{x}$, then (6.15) and (6.16) with $\bar{y}=$ $\Phi(\bar{x})$ present chain rules for the generalized subdifferentiation of compositions (6.2) in terms of the coderivative of $\Phi$ at $\bar{x}$. Using the scalarization formula proved in Section 5 , we obtain the following chain rules involving only subdifferentials.

6.3. Corollary. Let $X$ and $Y$ be Asplund spaces, let $\Phi: X \rightarrow Y$ be strictly Lipshitzian at $\bar{x}$, let $\varphi: Y \rightarrow \overline{\mathbf{R}}$ be normally compact around $\bar{y}=\Phi(\bar{x})$, and let the qualification condition

$$
\partial^{\infty} \varphi(\bar{y}) \cap \operatorname{ker} \partial\langle\cdot, \Phi\rangle(\bar{x})=\{0\}
$$

hold. Then one has the inclusions

$$
\begin{gathered}
\partial(\varphi \circ \Phi)(\bar{x}) \subset \bigcup_{y^{\star} \in \partial \varphi(\bar{y})} \partial\left\langle y^{\star}, \Phi\right\rangle(\bar{x}), \\
\partial^{\infty}(\varphi \circ \Phi)(\bar{x}) \subset \bigcup_{y^{\star} \in \partial^{\infty} \varphi(\bar{y})} \partial\left\langle y^{\star}, \Phi\right\rangle(\bar{x}) .
\end{gathered}
$$

Moreover, $\varphi \circ \Phi$ is subdifferential regular at $\bar{x}$, and (6.17) becomes an equality if $\varphi$ is subdifferentially regular at $\bar{y}$ and either $\Phi$ is strictly differentiable at $\bar{x}$ or the graph of $\Phi$ is regular at $(\bar{x}, \bar{y})$ with $\operatorname{dim} Y<\infty$.

Proof. This follows directly from Theorems 5.2 and 6.1 by taking into account Remark 6.2.

6.4. Remark. The chain rules obtained generalize previous results known in this direction. Note that Kruger [31] proved inclusion (6.17) when $X$ has a Fréchet differentiable renorm, $\operatorname{dim} Y<\infty, \Phi$ and $\varphi$ are, respectively, locally and directionally Lipschitzian. Jourani and Thibault [29] provided a different proof of (6.17) when $X$ is reflexive, $Y$ admits a Fréchet differentiable renorm, $\varphi$ is locally Lipschitzian, and $\Phi$ is compactly Lipschitzian at $\bar{x}$; cf. the remark before Theorem 5.2. Ioffe $[22,24]$ established chain rules for approximate subdifferentials in finite and infinite dimensions which coincide with (6.17) when, in particular, both $X$ and $Y$ are reflexive, $\varphi$ and $\Phi$ are locally Lipschitzian, and $\Phi$ has a strict prederivative at $\bar{x}$; see also Section 9. Recently Jourani and Thibault [30] extended the latter results for $G$-subdifferentials in Banach spaces to the case of compactly epi-Lipschitz functions $\varphi$ and strongly compactly Lipschitzian mappings $\Phi$.

Next let us consider a special class of marginal functions (6.1) where $\varphi$ is strictly differentiable at reference points. For this class we obtain refinements of the results in Theorem 6.1 and Corollary 6.3 that, in particular, ensure a subdifferential chain rule in the form of equality with no regularity and/or strictly Lipschitzness assumptions in infinite dimensions; see $[44,49]$ for finite dimensional counterparts. 
6.5. Theorem. Let $X$ and $Y$ be Asplund spaces. Assume that $\Phi: X \rightarrow Y$ is single-valued and Lipschitz continuous around $\bar{x}$ and $\varphi: X \times Y \rightarrow \mathbf{R}$ is strictly differentiable at $(\bar{x}, \Phi(\bar{x}))$. Then for $m(x)=\varphi(x, \Phi(x))$ one has

$$
\partial m(\bar{x})=\varphi_{x}^{\prime}(\bar{x}, \bar{y})+\partial\left\langle\varphi_{y}^{\prime}(\bar{x}, \bar{y}), \Phi\right\rangle(\bar{x}) \text { with } \bar{y}=\Phi(\bar{x}) .
$$

Proof. Let $x^{\star} \in \partial m(\bar{x})$, i.e., there are sequences $x_{k} \rightarrow \bar{x}$ and $x_{k}^{\star} \stackrel{w^{\star}}{\rightarrow} x^{\star}$ as $k \rightarrow \infty$ such that $x_{k}^{\star} \in \hat{\partial} m\left(x_{k}\right)$ for all $k$; see representation (2.16). Now we use the strict differentiablity of $\varphi$ at $(\bar{x}, \bar{y})$ similarly to the proof of equality (6.11) in Theorem 6.1. To this end, for any sequence $\gamma_{\nu} \downarrow 0$ we find $\eta_{\nu} \downarrow 0$ and $k_{\nu} \rightarrow \infty$ as $\nu \rightarrow \infty$ with

$$
\begin{aligned}
& \left\langle\varphi_{y}^{\prime}(\bar{x}, \bar{y}), \Phi(x)-\Phi\left(x_{k_{\nu}}\right)\right\rangle-\left\langle x_{k_{\nu}}^{\star}-\varphi_{x}^{\prime}(\bar{x}, \bar{y}), x-x_{k_{\nu}}\right\rangle \\
& \quad \geq-\gamma_{\nu}(1+l)\left\|x-x_{k_{\nu}}\right\| \forall x \in B_{\eta_{\nu} / 2}\left(x_{k_{\nu}}\right), \nu=1,2, \ldots,
\end{aligned}
$$

where $l \geq 0$ is a Lipschitz modulus of $\Phi$ around $\bar{x}$. It follows from (6.19) and (2.11) that

$$
x_{k_{\nu}}^{\star}-\varphi_{x}^{\prime}(\bar{x}, \bar{y}) \in \tilde{\partial}_{\varepsilon_{\nu}}\left\langle\varphi_{y}^{\prime}(\bar{x}, \bar{y}), \Phi\right\rangle\left(x_{k_{\nu}}\right) \text { with } \varepsilon_{\nu}:=\gamma_{\nu}(1+l) .
$$

The latter implies the inclusion " $\subset$ " in $(6.18)$ by virtue of (2.12). To justify the opposite inclusion we employ the same arguments starting with a point $x^{\star} \in$ $\partial\left\langle\varphi_{y}^{\prime}(\bar{x}, \bar{y}), \Phi\right\rangle(\bar{x})$. This ends the proof of the theorem.

6.6. Remark. If $\Phi$ is not strictly differentiable at $\bar{x}$, then equality (6.18) does not follow from the equality conclusions of Theorem 6.1 even for finite dimensional spaces $X$ and $Y$. On the other hand, the assumptions of Theorem 6.5 do not ensure the subdifferential regularity of the composite function. Note also that we get a subdifferential chain rule in (6.18) although $\Phi$ is not assumed to be strictly Lipschitzian at $\bar{x}$ as in Corollary 6.3.

Let us present another set of conditions that provide equality in the subdifferentiation formula (6.5) with no regularity. The next theorem also contains a new equality chain rule for the singular subdifferentials (2.8).

6.7. Theorem. (i) Let $X$ and $Y$ be Asplund spaces, let $\Phi: X \rightarrow Y$ be strictly differentiable at $\bar{x}$ with $\Phi^{\prime}(\bar{x})$ invertible (i.e., surjective and one-to-one), and let $\varphi: X \times Y \rightarrow \overline{\mathbf{R}}$ be represented as

$$
\varphi(x, y)=\varphi_{1}(x)+\varphi_{2}(y)
$$

where $\varphi_{1}$ is strictly differentiable at $\bar{x}$ and $\varphi_{2}$ is l.s.c. around $\bar{y}=\Phi(\bar{x})$. Then

$$
\partial m(\bar{x})=\varphi_{1}^{\prime}(\bar{x})+\left(\Phi^{\prime}(\bar{x})\right)^{\star} \partial \varphi_{2}(\bar{y}) .
$$

(ii) If in assumptions (i) $\varphi_{1}$ is Lipschitz continuous around $\bar{x}$, then

$$
\partial^{\infty} m(\bar{x})=\left(\Phi^{\prime}(\bar{x})\right)^{\star} \partial^{\infty} \varphi_{2}(\bar{y})
$$

Proof. Observe that

$$
m(x)=\varphi(x, \Phi(x))=\varphi_{1}(x)+\varphi_{2}(\Phi(x)) .
$$

If $\varphi_{1}$ is strictly differentiable at $\bar{x}$, one has

$$
\partial m(\bar{x})=\varphi_{1}^{\prime}(\bar{x})+\partial\left(\varphi_{2} \circ \Phi\right)(\bar{x})
$$

according to Corollary 4.3(i). Let us prove that

$$
\partial\left(\varphi_{2} \circ \Phi\right)(\bar{x})=\left(\Phi^{\prime}(\bar{x})\right)^{\star} \partial \varphi_{2}(\bar{y}) \text { with } \bar{y}=\Phi(\bar{x})
$$


if $\Phi$ is strictly differentiable at $\bar{x}$ and $\Phi^{\prime}(\bar{x})$ is invertible. First we justify the inclusion " $\supset$ " in (6.22). Remember that due to Leach [36] the inverse mapping $\Phi^{-1}$ is locally single-valued and strictly differentiable at $\Phi(\bar{x})$ with the strict derivative $\left(\Phi^{\prime}(\bar{x})\right)^{-1}$ at this point.

Let $y^{\star} \in \partial \varphi_{2}(\bar{y})$. Employing (2.16), one has sequences $y_{k} \rightarrow \bar{y}$ and $y_{k}^{\star} \stackrel{w^{\star}}{\rightarrow} y^{\star}$ as $k \rightarrow \infty$ with $y_{k}^{\star} \in \hat{\partial} \varphi_{2}\left(y_{k}\right)$ for all $k$. Now using the fact that $\Phi$ is a local homeomorphism around $\bar{x}$ and the procedure similar to the proofs of equalities (6.11) and (6.18) in Theorems 6.1 and 6.5, for any sequence $\gamma_{\nu} \downarrow 0$ we find $\eta_{\nu} \downarrow$ $0, x_{\nu} \rightarrow \bar{x}$ and a subsequence $k_{\nu} \rightarrow \infty$ as $\nu \rightarrow \infty$ such that

$$
\begin{aligned}
\varphi_{2}(\Phi(x))-\varphi_{2}\left(\Phi\left(x_{\nu}\right)\right)-\left\langle\left(\Phi^{\prime}(\bar{x})\right)^{\star} y_{k_{\nu}}^{\star}, x-x_{\nu}\right\rangle \geq-\varepsilon_{\nu} & \left\|x-x_{\nu}\right\| \\
\forall x & \in B_{\eta_{\nu}}\left(x_{\nu}\right), \nu=1,2, \ldots,
\end{aligned}
$$

where $\varepsilon_{\nu}:=\gamma_{\nu} \cdot\left\|y_{k_{\nu}}^{\star}\right\|$. By virtue of (2.11) and (2.12) the latter implies the inclusion " $\supset$ " in (6.22).

To justify the opposite inclusion we represent $\varphi_{2}$ in the form

$$
\varphi_{2}(y)=\left(\psi \circ \Phi^{-1}\right)(y) \text { with } \psi(x):=\left(\varphi_{2} \circ \Phi\right)(x) .
$$

Applying the inclusion " $\supset$ " in (6.22) to the composition $\psi \circ \Phi$ and taking into account that $\left(\Phi^{-1}\right)^{\prime}(\bar{y})=\left(\Phi^{\prime}(\bar{x})\right)^{-1}$, we obtain the inclusion " $\subset$ " in (6.22). This proves formula (6.20).

To establish (ii) we observe that $\partial^{\infty} m(\bar{x})=\partial^{\infty}\left(\varphi_{2} \circ \Phi\right)(\bar{x})$ if $\varphi_{1}$ is Lipschitz continuous around $\bar{x}$; see Corollary 4.3(ii). The proof of

$$
\partial^{\infty}\left(\varphi_{2} \circ \Phi\right)(\bar{x})=\left(\Phi^{\prime}(\bar{x})\right)^{\star} \partial^{\infty} \varphi_{2}(\Phi(\bar{x}))
$$

is similar to $(6.22)$ by using the last representation in (2.17). We complete the proof of the theorem.

6.8. Remarks. (a) For $\varphi(x, y)=\varphi(y)$, the results presented in Theorems 6.5 and 6.7 provide new chain rules with equality for the generalized differentiation of compositions (6.2) in infinite dimensions. They have many useful consequences and applications, some of which are considered in the next section.

(b) Theorems 6.5 and 6.7 and some related results for the sequential subdifferentials under consideration can be obtained in general Banach spaces. The basic framework for this development is the limiting representation (2.12), where $\varepsilon>0$ cannot be dismissed like in the case of Asplund spaces. We refer to [52] for more details.

Now let us apply the chain rules obtained to evaluate the normal cone (2.2) to sets given in the form

$$
\Phi^{-1}(\Lambda):=\{x \in X \mid \Phi(x) \in \Lambda\}
$$

where the mapping $\Phi: X \rightarrow Y$ is continuous around $\bar{x}$ and the set $\Lambda \subset Y$ is closed.

6.9. Corollary. Let $X$ and $Y$ be Asplund spaces, let $\Lambda$ be normally compact around $\Phi(\bar{x})$, and let the qualification condition

$$
N(\Phi(\bar{x}) ; \Lambda) \cap \operatorname{ker} D^{\star} \Phi(\bar{x})=\{0\}
$$

hold. Then one has the inclusion

$$
N\left(\bar{x} ; \Phi^{-1}(\Lambda)\right) \subset \bigcup\left[D^{\star} \Phi(\bar{x})\left(y^{\star}\right) \mid y^{\star} \in N(\Phi(\bar{x}) ; \Lambda)\right],
$$


where $D^{\star} \Phi(\bar{x})\left(y^{\star}\right)$ can be replaced by $\partial\left\langle y^{\star}, \Phi\right\rangle(\bar{x})$ if $\Phi$ is strictly Lipschitzian at $\bar{x}$. In addition, the set $\Phi^{-1}(\Lambda)$ is regular at $\bar{x}$, and equality holds in (6.23) if $\Lambda$ is regular at $\Phi(\bar{x})$ and either assumptions (a) or (b) of Theorem 6.1 are fulfilled. Moreover, one has the equality

$$
N\left(\bar{x} ; \Phi^{-1}(\Lambda)\right)=\left(\Phi^{\prime}(\bar{x})\right)^{\star} N(\Phi(\bar{x}) ; \Lambda)
$$

if $\Phi$ is strictly differentiable at $\bar{x}$ and $\Phi^{\prime}(\bar{x})$ is invertible.

Proof. The first part of these results related to (6.23) follows from Theorem 6.1 and Corollary 6.3 with $\varphi(x, y)=\delta(y, \Lambda)$. Equality (6.24) is implied by Theorem 6.7(i).

6.10. Remark. Using calculus results, we can obtain various applications and developments of the subdifferentiation formulas for marginal functions in the case of special representations of the multifunction $\Phi$ in (6.1). One of the most important classes of multifunctions arising in nonlinear analysis and optimization is represented in the form of the so-called parametric constraint systems

$$
\Phi(x)=\{y \in Y \mid g(x, y) \in \Lambda,(x, y) \in \Omega\},
$$

where $g: X \times Y \rightarrow Z$ is a continuous mapping between Banach spaces. Among others, this class includes multi-valued solution maps to parametric generalized equations and variational inequalities; see, e.g., [47, 48, 61] and references therein. Employing the results in Corollaries 4.5 and 6.9, one can compute the coderivative of $\Phi$ in (6.25) and get effective representations of the marginal function subdifferentials in terms of the normal and differential constructions for $g$ and $\Lambda$; cf. [47, 48] for the case of finite dimensions. Developing this approach and using the corresponding criteria in $[50,53]$, we are able to obtain various results on metric regularity and Lipschitzian stability of parametric constraint and variational systems in infinite dimensions; see [53].

\section{More CAlculus}

In this section we consider extended-real-valued functions defined on Asplund spaces and prove calculus results related to the generalized differentiation of products, quotients, maxima, minima as well as partial derivatives and mean values. The results obtained are based on the chain rules in Section 6 .

Let us start with product rules. For simplicity, we consider the case of locally Lipschitzian functions when the product rules obtained involve only subdifferentials, without using coderivatives as in the general case.

7.1. Theorem. Let $X$ be an Asplund space and let $\varphi_{i}: X \rightarrow \mathbf{R}, i=1,2$, be Lipschitz continuous around $\bar{x}$. Then one has product rules of the equality form

$$
\partial\left(\varphi_{1} \cdot \varphi_{2}\right)(\bar{x})=\partial\left(\varphi_{2}(\bar{x}) \varphi_{1}+\varphi_{1}(\bar{x}) \varphi_{2}\right)(\bar{x})
$$

and of the inclusion form

$$
\partial\left(\varphi_{1} \cdot \varphi_{2}\right)(\bar{x}) \subset \partial\left(\varphi_{2}(\bar{x}) \varphi_{1}\right)(\bar{x})+\partial\left(\varphi_{1}(\bar{x}) \varphi_{2}\right)(\bar{x})
$$

which becomes an equality if one of the functions $\varphi_{i}, i=1,2$, is strictly differentiable at $\bar{x}$. Moreover, equality holds in (7.2), and $\varphi_{1} \cdot \varphi_{2}$ is subdifferentially regular at $\bar{x}$ when both functions $\varphi_{2}(\bar{x}) \varphi_{1}$ and $\varphi_{1}(\bar{x}) \varphi_{2}$ are subdifferentially regular at this point. 
Proof. To establish (7.1) we employ equality (6.18) in Theorem 6.5 with $\Phi: X \rightarrow$ $\mathbf{R}^{2}$ and $\varphi: \mathbf{R}^{2} \rightarrow \mathbf{R}$ defined by $\Phi(x):=\left(\varphi_{1}, \varphi_{2}\right)$ and $\varphi\left(y_{1}, y_{2}\right):=y_{1} \cdot y_{2}$. Inclusion (7.2) and the other statements of the theorem follow from (7.1) due to Theorem 4.1 and Corollary 4.3(i).

7.2. Remark. The results obtained generalize the classical product rule to the case of nonsmooth functions. Note that the right-hand side of (7.2) is equal to

$$
\varphi_{2}(\bar{x}) \partial \varphi_{1}(\bar{x})+\varphi_{1}(\bar{x}) \partial \varphi_{2}(\bar{x})
$$

if $\varphi_{i}(\bar{x}) \geq 0, i=1,2$. This follows from the obvious equality $\partial(\alpha \varphi)(\bar{x})=\alpha \partial \varphi(\bar{x})$, valid for any $\varphi$ and $\alpha \geq 0$. Furthermore, one always has

$$
\partial(\alpha \varphi)(\bar{x})=\alpha \partial^{+} \varphi(\bar{x}) \text { for } \quad \alpha<0
$$

where $\partial^{+} \varphi(\bar{x}):=-\partial \varphi(\bar{x})$ is the superdifferential of $\varphi$ at $\bar{x}$ that is considerably different from the subdifferential (2.7) for nonsmooth functions; see, e.g., [44].

The next theorem provides useful quotient rules for subdifferentials of locally Lipschitzian functions.

7.3. Theorem. Let $\varphi_{2}(\bar{x}) \neq 0$ under the general assumptions of the previous theorem. Then one has quotient rules of the equality form

$$
\left.\partial\left(\varphi_{1} / \varphi_{2}\right)(\bar{x})=\left[\partial\left(\varphi_{2}(\bar{x}) \varphi_{1}\right)-\varphi_{1}(\bar{x}) \varphi_{2}\right)(\bar{x})\right] /\left[\varphi_{2}(\bar{x})\right]^{2}
$$

and of the inclusion form

$$
\partial\left(\varphi_{1} / \varphi_{2}\right)(\bar{x}) \subset\left[\partial\left(\varphi_{2}(\bar{x}) \varphi_{1}\right)(\bar{x})-\partial\left(\varphi_{1}(\bar{x}) \varphi_{2}\right)(\bar{x})\right] /\left[\varphi_{2}(\bar{x})\right]^{2},
$$

where equality holds when one of the functions $\varphi_{i}, i=1,2$, is strictly differentiable at $\bar{x}$. Furthermore, $\varphi_{1} / \varphi_{2}$ is subdifferentially regular at $\bar{x}$, and (7.4) becomes an equality if both functions $\varphi_{2}(\bar{x}) \varphi_{1}$ and $-\varphi_{1}(\bar{x}) \varphi_{2}$ are subdifferentially regular at this point.

Proof. This is proved similarly to Theorem 7.1, using the representation $\left(\varphi_{1} / \varphi_{2}\right)(x)$ $=(\varphi \circ \Phi)(x)$, where $\Phi: X \rightarrow \mathbf{R}^{2}$ and $\varphi: \mathbf{R}^{2} \rightarrow \mathbf{R}$ are defined by

$$
\Phi(x):=\left(\varphi_{1}(x), \varphi_{2}(x)\right) \text { and } \varphi\left(y_{1}, y_{2}\right):=y_{1} / y_{2} \text {. }
$$

7.4. Corollary. Let $X$ be an Asplund space and let $\varphi: X \rightarrow \mathbf{R}$ be Lipschitz continuous around $\bar{x}$ with $\varphi(\bar{x}) \neq 0$. Then one has

$$
\partial(1 / \varphi)(\bar{x})=-\partial^{+} \varphi(\bar{x}) / \varphi^{2}(\bar{x}) .
$$

Proof. This follows from the quotient rule (7.3) with $\varphi_{1}=1$ and $\varphi_{2}=\varphi$.

Now we consider the maximum function of the form

$$
\left(\bigvee \varphi_{j}\right)(x):=\max \left\{\varphi_{j}(x) \mid j=1, \ldots, n\right\}, n \geq 2,
$$

where $\varphi_{j}: X \rightarrow \overline{\mathbf{R}}$ for $j=1, \ldots, n$. Let us construct the sets

$$
J(\bar{x}):=\left\{j \in\{1, \ldots, n\} \mid \varphi_{j}(\bar{x})=\left(\bigvee \varphi_{j}\right)(\bar{x})\right\},
$$

$\Lambda(\bar{x}):=\left\{\left(\lambda_{1}, \ldots, \lambda_{n}\right) \in \mathbf{R}^{n} \mid \lambda_{j} \geq 0, \lambda_{1}+\cdots \lambda_{n}=1, \lambda_{j}\left(\varphi_{j}(\bar{x})-\left(\bigvee \varphi_{j}\right)(\bar{x})\right)=0\right\}$.

The following theorem generalizes and extends in various directions the corresponding results in $[22,24,31,43,44]$. 
7.5. Theorem. (i) Let $X$ be Asplund and let $\varphi_{j}$ be l.s.c. around $\bar{x}$ for $j \in J(\bar{x})$ and upper semicontinuous at $\bar{x}$ for $j \notin J(\bar{x})$. Assume that the functions $\varphi_{j}$ are normally compact around $\bar{x}$ for all but one $j \in J(\bar{x})$ and the qualification condition (4.1) considered for $j \in J(\bar{x})$ is fulfilled. Then

$$
\begin{gathered}
\partial\left(\bigvee \varphi_{j}\right)(\bar{x}) \subset \bigcup\left\{\sum_{j \in J(\bar{x})} \lambda_{j} \circ \partial \varphi_{j}(\bar{x}) \mid\left(\lambda_{1}, \ldots, \lambda_{n}\right) \in \Lambda(\bar{x})\right\}, \\
\partial^{\infty}\left(\bigvee \varphi_{j}\right)(\bar{x}) \subset \sum_{j \in J(\bar{x})} \partial^{\infty} \varphi_{j}(\bar{x}),
\end{gathered}
$$

where we define $\lambda \circ \partial \varphi(x)$ by $\lambda \partial \varphi(x)$ for $\lambda>0$ and by $\partial^{\infty} \varphi(x)$ for $\lambda=0$. Moreover, the maximum function (7.5) is subdifferentially regular at $\bar{x}$, and equality holds in (7.6) if the sets epi $\varphi_{j}$ are regular at $\left(\bar{x}, \varphi_{j}(\bar{x})\right)$ for $j \in J(\bar{x})$.

(ii) Let $X$ be Asplund and let $\varphi_{j}, j=1, \ldots, n$, be Lipschitz continuous around $\bar{x}$. Then one has the inclusions

$$
\begin{aligned}
& \partial\left(\bigvee \varphi_{j}\right)(\bar{x}) \subset \bigcup\left\{\partial\left(\sum_{j \in J(\bar{x})} \lambda_{j} \varphi_{j}\right)(\bar{x}) \mid\left(\lambda_{1}, \ldots, \lambda_{n}\right) \in \Lambda(\bar{x})\right\} \\
& \subset \operatorname{co}\left\{\partial \varphi_{j}(\bar{x}) \mid j \in J(\bar{x})\right\}
\end{aligned}
$$

where equality holds and the maximum function is subdifferentially regular at $\bar{x}$ if the functions $\varphi_{j}$ are subdifferentially regular at $\bar{x}$ for $j \in J(\bar{x})$.

Proof. First we prove inclusion (7.6), denoting the maximum function (7.5) by $g(x)$. Let $x^{\star} \in \partial g(\bar{x})$, i.e., $\left(x^{\star},-1\right) \in N((\bar{x}, g(\bar{x}))$; epi $g)$. We observe that epi $g=\bigcap_{j=1}^{n}\left(\right.$ epi $\left.\varphi_{j}\right)$, and $(\bar{x}, g(\bar{x}))$ is an interior point of the set epi $\varphi_{j}$ for any $j \notin J(\bar{x})$ due to the upper semicontinuity assumption. Taking this into account and also that the space $X \times \mathbf{R}$ is Asplund, one can apply Corollary 4.5 to the above normal cone, considering there only $j \in J(\bar{x})$. It is clear that the qualification condition (4.17) in Corollary 4.5 is reduced to (4.1) with $j \in J(\bar{x})$ for the case considered. In this way, we represent $\left(x^{\star},-1\right)$ as a sum of $\left(x_{j}^{\star},-\lambda_{j}\right) \in N\left(\left(\bar{x}, \varphi_{j}(\bar{x})\right)\right.$; epi $\left.\varphi_{j}\right)$ with $j \in J(\bar{x})$ and $\left(\lambda_{1}, \ldots, \lambda_{n}\right) \in \Lambda(\bar{x})$. The latter implies (7.6) according to the definitions.

To prove (7.7) we use the same procedure based on Corollary 4.5. The equality and regularity statements in (i) follow from the corresponding conclusions in Corollary 4.5 and the fact that the regularity of epi $g$ at $(\bar{x}, g(\bar{x}))$ always implies the subdifferential regularity of (7.5) at $\bar{x}$.

Now let us prove (ii). To do this we observe that the maximum function (7.5) is represented as a composition $(\varphi \circ \Phi)(x)$ with Lipschitz continuous mappings $\Phi: X \rightarrow \mathbf{R}^{n}$ and $\varphi: \mathbf{R}^{n} \rightarrow \mathbf{R}$ defined by

$$
\Phi(x):=\left(\varphi_{1}(x), \ldots, \varphi_{n}(x)\right) \text { and } \varphi\left(y_{1}, \ldots, y_{n}\right):=\max \left\{y_{j} \mid j=1, \ldots, n\right\} .
$$

Note that the function $\varphi$ defined is convex and its subdifferential is well known in convex analysis; see. e.g., [10]. Now employing Corollary 6.3, we get the first inclusion in (7.8), that implies the second one by virtue of Corollary 4.3. Note that if $\varphi_{j}, j \in J(\bar{x})$, are Lipschitz continuous around $\bar{x}$, then the second inclusion in (7.8) coincides with (7.7). Moreover, in this case the regularity of epi $\varphi_{j}$ at $\left(\bar{x}, \varphi_{j}(\bar{x})\right)$ is equivalent to the subdifferential regularity of $\varphi_{j}$ at $\bar{x}$. Therefore, the regularity and equality statements in (ii) follows from the corresponding results in (i). This ends the proof of the theorem. 
Now we consider the minimum function

$$
\left(\bigwedge \varphi_{j}\right)(x):=\min \left\{\varphi_{j}(x) \mid j=1, \ldots, n\right\}, \quad n \geq 2,
$$

for $\varphi_{j}: X \rightarrow \overline{\mathbf{R}}$. Note that (7.9) and (7.5) are essentially different from the viewpoint of the unilateral subdifferentials (2.7) and (2.8). Denoting $J(x):=\{j \in$ $\left.\{1, \ldots, n\} \mid \varphi_{j}(x)=\left(\bigwedge \varphi_{j}\right)(\bar{x})\right\}$, one can obtain the following subdifferentiation formulas for (7.9) similarly to the finite dimensional case; cf. [22, 44].

7.6. Theorem. Let $X$ be an Asplund space. Assume that the functions $\varphi_{j}$ and (7.9) are l.s.c. around $\bar{x}$ for $j \in J(\bar{x})$ and $\varphi_{j}$ are l.s.c. at this point for $j \notin J(\bar{x})$. Then one has

$$
\begin{gathered}
\partial\left(\bigwedge \varphi_{j}\right)(\bar{x}) \subset \bigcup\left\{\partial \varphi_{j}(\bar{x}) \mid j \in J(\bar{x})\right\}, \\
\partial^{\infty}\left(\bigwedge \varphi_{j}\right)(\bar{x}) \subset \bigcup\left\{\partial^{\infty} \varphi_{j}(\bar{x}) \mid j \in J(\bar{x})\right\} .
\end{gathered}
$$

Proof. To prove (7.10) let us pick a sequence $\left\{x_{k}\right\} \subset X$ such that $x_{k} \rightarrow \bar{x}$ and $\varphi_{j}\left(x_{k}\right) \rightarrow\left(\bigwedge \varphi_{j}\right)(\bar{x})$ for $j \in J(\bar{x})$ as $k \rightarrow \infty$. Using the 1.s.c. of $\varphi_{j}$ at $\bar{x}$ for $j \notin J(\bar{x})$, we get $J\left(x_{k}\right) \subset J(\bar{x})$. According to (2.11) with $\varepsilon=0$ this yields

$$
\hat{\partial}\left(\bigwedge \varphi_{j}\right)\left(x_{k}\right) \subset \bigcup\left\{\hat{\partial} \varphi_{j}\left(x_{k}\right) \mid j \in J(\bar{x})\right\} \quad \forall k=1,2, \ldots
$$

The latter implies (7.10) due to representation (2.16) in Theorem 2.9(iii). To prove (7.11) we use similar arguments based on representation (2.17).

Next we apply chain rules to obtain connections between "full" and "partial" subdifferentials for functions of many variables. Let $\varphi: X \times Y \rightarrow \overline{\mathbf{R}}$ be finite at $(\bar{x}, \bar{y})$ and let $\partial_{x} \varphi(\bar{x}, \bar{y})$ and $\partial_{x}^{\infty} \varphi(\bar{x}, \bar{y})$ be, respectively, its partial subdifferential and partial singular subdifferential in $x$ at this point, i.e., the corresponding subdifferentials $(2.7)$ and $(2.8)$ of the function $\varphi(\cdot, \bar{y})$ at $\bar{x}$. The following theorem (cf. $[39,44,60]$ in finite dimensions) establishes the relationships between the partial subdifferentials under consideration and projections of their full counterparts.

7.7. Theorem. Let $X$ and $Y$ be Asplund spaces, and let $\varphi: X \times Y \rightarrow \overline{\mathbf{R}}$ be l.s.c. and normally compact around $(\bar{x}, \bar{y})$. Under the qualification condition

$$
\nexists y^{\star} \neq 0 \text { with }\left(0, y^{\star}\right) \in \partial^{\infty} \varphi(\bar{x}, \bar{y})
$$

one has the inclusions

$$
\begin{gathered}
\partial_{x} \varphi(\bar{x}, \bar{y}) \subset\left\{x^{\star} \in X^{\star} \mid\left(x^{\star}, y^{\star}\right) \in \partial \varphi(\bar{x}, \bar{y}) \text { for some } y^{\star} \in Y^{\star}\right\}, \\
\partial_{x}^{\infty} \varphi(\bar{x}, \bar{y}) \subset\left\{x^{\star} \in X^{\star} \mid\left(x^{\star}, y^{\star} \in \partial^{\infty} \varphi(\bar{x}, \bar{y}) \text { for some } y^{\star} \in Y^{\star}\right\} .\right.
\end{gathered}
$$

Moreover, $\varphi(\cdot, \bar{y})$ is subdifferentially regular at $\bar{x}$, and (7.12) becomes an equality if $\varphi$ is subdifferentially regular at $(\bar{x}, \bar{y})$.

Proof. We can represent $\varphi(x, \bar{y})=(\varphi \circ \Phi)(x)$, where $\Phi: X \rightarrow X \times Y$ is a smooth mapping defined by $\Phi(x):=(x, \bar{y})$. Then all the results of the theorem follow from Corollary 6.3 .

In conclusion of this section we employ the chain rule (6.17) to obtain a nonsmooth generalization of the classical mean value theorem in terms of the sequential subdifferential constructions in Asplund spaces. For any function $\varphi: X \rightarrow \overline{\mathbf{R}}$ we define the symmetric subdifferential

$$
\partial^{0} \varphi(\bar{x}):=\partial \varphi(\bar{x}) \cup \partial^{+} \varphi(\bar{x})=\partial \varphi(\bar{x}) \cup[-\partial(-\varphi)(\bar{x})]
$$


at $\bar{x}$, which is a nonconvex subset of $X^{\star}$ with the symmetry property $\partial^{0}(-\varphi)(\bar{x})=$ $-\partial^{0} \varphi(\bar{x})$. The following nonsmooth analogue of the Fermat stationary principle is useful in the sequel.

7.8. Proposition. Let $X$ be a Banach space, let $\varphi: X \rightarrow \overline{\mathbf{R}}$, and let $\bar{x} \in \operatorname{dom} \varphi$. Then $0 \in \partial \varphi(\bar{x})$ if $\varphi$ has a local minimum at $\bar{x}$, and $0 \in \partial^{+} \varphi(\bar{x})$ if $\bar{x}$ has a local maximum at $\bar{x}$. So $0 \in \partial^{0} \varphi(\bar{x})$ if $\bar{x}$ is either a minimum or a maximum point of $\varphi$.

Proof. If $\bar{x}$ is a minimum point of $\varphi$ then $0 \in \hat{\partial} \varphi(\bar{x}) \subset \partial \varphi(\bar{x})$. This follows directly from (2.11) and implies the other statements in the proposition according to the definitions.

To formulate the mean value theorem we denote $\partial^{\infty,+} \varphi(\bar{x}):=-\partial^{\infty}(-\varphi)(\bar{x})$, $(b-a)^{\perp}:=\left\{x^{\star} \in X^{\star} \mid\left\langle x^{\star}, b-a\right\rangle=0\right\},[a, b]:=\{a+t(b-a) \mid 0 \leq t \leq 1\}$, and similarly $(a, b),[a, b),(a, b]$ for any $a, b \in X$.

7.9. Theorem. Let $X$ be Asplund and let $\varphi: X \rightarrow \overline{\mathbf{R}}$ be continuous in $[a, b] \subset X$. Assume that both $\varphi$ and $-\varphi$ are normally compact around every point in $(a, b)$ and

$$
\left[\partial^{\infty} \varphi(x) \cup \partial^{\infty,+} \varphi(x)\right] \cap(b-a)^{\perp}=\{0\} \forall x \in(a, b) .
$$

Then there is a point $c \in(a, b)$ such that

$$
\varphi(b)-\varphi(a) \in\left\langle\partial^{0} \varphi(c), b-a\right\rangle .
$$

Proof. Let us consider the real-valued function

$$
f(t):=\varphi(a+t(b-a))+t(\varphi(a)-\varphi(b)), \quad 0 \leq t \leq 1,
$$

that is continuous in $[0,1]$ with $f(0)=f(1)=\varphi(a)$. Employing the classical Weierstrass theorem and excluding the trivial case $f(t) \equiv$ const, we find a point $\theta \in(0,1)$ where $f$ attains either its minimum or maximum on $[0,1]$. According to Proposition 7.8 one has $0 \in \partial^{0} f(\theta)$. Due to (7.16) the latter implies

$$
\varphi(b)-\varphi(a) \in \partial_{t}^{0} \varphi(a+\theta(b-a))
$$

where the right-hand side is the symmetric subdifferential (7.13) of the function $t \rightarrow \varphi(a+t(b-a))$ at $t=\theta$.

Now let us represent $\varphi(a+t(b-a))$ in the composition form

$$
\varphi(a+t(b-a))=(\varphi \circ \Phi)(t), \quad 0 \leq t \leq 1,
$$

with a smooth mapping $\Phi:[0,1] \rightarrow X$ defined by $\Phi(t):=a+t(b-a)$. We employ Corollary 6.3 to compute the symmetric subdifferential (7.13) of the composition (7.18), furnishing this separately for the subdifferential (2.7) and its superdifferential counterpart. Note that assumption (7.14) implies the qualification condition in Corollary 6.3 for both functions $\varphi$ and $-\varphi$. Therefore, we derive (7.15) from (7.17) with $c:=a+\theta(b-a)$.

7.10. Corollary. Let $X$ be an Asplund space and let $\varphi: U \rightarrow \mathbf{R}$ be Lipschitz continuous in some open set $U \subset X$ containing $[a, b]$. Then there exists $c \in(a, b)$ where (7.15) holds.

Proof. This follows from Theorem 7.9 by virtue of Proposition 2.5, that ensures (7.14). 
7.11. Remarks. (a) One cannot replace $\partial^{0} \varphi$ by $\partial \varphi$ in (7.15). A simple counterexample is provided by the function $\varphi(x)=-|x|$ defined on the real interval $[-1,1]$ with $\partial \varphi(0)=\{-1,1\}$.

(b) Theorem 7.9 extends the results in Mordukhovich [43, 44, 49] to infinite dimensions. Corollary 7.10 was obtained by Kruger [31] for Lipschitz continuous functions defined on spaces with Fréchet differentiable renorms; cf. also [40, 54].

(c) Some results in this section based on the chain rules in Theorems 6.5 and 6.7 hold true in any Banach spaces; cf. Remark 6.8(b) and the proofs furnished above. They include product and quotient rules in equality forms (7.1) and (7.3), minimum rules in Theorem 7.6, a version of the mean value theorem, etc. More details can be found in [52].

\section{Approximate mean value theorem and some applications}

This section is concerned with mean value results of a new type that are grouped around the so-called approximate mean value theorem. Results of this type were first obtained by Zagrodny [70] for subgradients of Clarke and related directional derivatives in Banach spaces. Here we present an advanced version of such results involving Fréchet subgradients of l.s.c. functions defined in Asplund spaces. We also consider some important applications of the results obtained, including relationships between our basic sequential constructions and Clarke's concepts of generalized normals and subgradients. We begin by formulating the following useful property of Fréchet subgradients, proved by Fabian [18, Lemma 3].

8.1. Proposition. Let $X$ be an Asplund space and let $\varphi_{i}: X \rightarrow(-\infty, \infty], i=1,2$, be two l.s.c. functions whose sum $\varphi_{1}+\varphi_{2}$ attains an isolated minimun at some $\bar{x}$. Given $\gamma>0$, we assume that the set $\left\{x \in B_{\gamma}(\bar{x}) \mid \varphi_{2}(x) \leq \alpha\right\}$ is norm compact for any $\alpha \in \mathbf{R}$. Then there are $x_{1}, x_{2} \in B_{\gamma}(\bar{x})$ with $\left|\varphi_{i}\left(x_{i}\right)-\varphi_{i}(\bar{x})\right| \leq \gamma, i=1,2$, such that

$$
0 \in \hat{\partial} \varphi_{1}\left(x_{1}\right)+\hat{\partial} \varphi_{2}\left(x_{2}\right) .
$$

Now let us prove the approximate mean value theorem as follows.

8.2. Theorem. Let $X$ be an Asplund space and let $\varphi: X \rightarrow(-\infty, \infty]$ be a l.s.c. function finite at two given points $a \neq b$. Then for any point $c \in[a, b)$ satisfying

$$
\varphi(c)+\frac{\varphi(b)-\varphi(a)}{\|b-a\|}\|c-b\| \leq \varphi(x)+\frac{\varphi(b)-\varphi(a)}{\|b-a\|}\|x-b\| \forall x \in[a, b]
$$

there exist sequences $x_{k} \stackrel{\varphi}{\rightarrow}$ c and $x_{k}^{\star} \in \hat{\partial} \varphi\left(x_{k}\right)$ such that

$$
\begin{gathered}
\liminf _{k \rightarrow \infty}\left\langle x_{k}^{\star}, b-x_{k}\right\rangle \geq \frac{\varphi(b)-\varphi(a)}{\|b-a\|}\|b-c\|, \\
\liminf _{k \rightarrow \infty}\left\langle x_{k}^{\star}, b-a\right\rangle \geq \varphi(b)-\varphi(a) .
\end{gathered}
$$

Moreover, when $c \neq a$ one has

$$
\lim _{k \rightarrow \infty}\left\langle x_{k}^{\star}, b-a\right\rangle=\varphi(b)-\varphi(a) .
$$

Proof. Let us denote by $f(x)$ the function on the right-hand side of (8.1). This function is l.s.c. and, therefore, attains its minimum over the compact set $[a, b]$ at some point $c \in[a, b]$. Due to $f(a)=f(b)=\varphi(a)$ one can always take $c \in[a, b)$, i.e., 
the set of points $c$ satisfying (8.1) is nonempty. Moreover, for any given $c$ in (8.1) and each $k=1,2, \ldots$ the function

$$
f_{k}(x):=\varphi(x)+\frac{\|x-b\|}{\|b-a\|}(\varphi(b)-\varphi(a))+\frac{1}{k}\|x-c\|+\delta(x,[a, b])
$$

attains an isolated local minimum at $c$.

Let $\varphi_{1}:=\varphi$ and let $\varphi_{2}$ be the sum of the last three functions in (8.5). One can check that all the assumptions of Proposition 8.1 hold. Taking there $\bar{x}=c$ and $\gamma=1 / k$, we find $x_{k} \stackrel{\varphi}{\rightarrow} c$ and $y_{k} \rightarrow c$ as $k \rightarrow \infty$ such that $0 \in \hat{\partial} \varphi\left(x_{k}\right)+\hat{\partial} \varphi_{2}\left(y_{k}\right)$ for all $k=1,2, \ldots$ Observe that each term of $\varphi_{2}$ is convex and the first two terms are continuous. Employing the Moreau-Rockafellar subdifferential theorem in convex analysis for $\hat{\partial} \varphi_{2}\left(y_{k}\right)=\partial \varphi_{2}\left(y_{k}\right)$ and taking into account the well-known subdifferentiation formulas for the convex functions in (8.5) at $y_{k} \neq b$, we find elements $x_{k}^{\star}, u_{k}^{\star}, v_{k}^{\star}$, and $w_{k}^{\star}$ in $X^{\star}$ such that $x_{k}^{\star} \in \hat{\partial} \varphi\left(x_{k}\right), w_{k}^{\star} \in(1 / k) B^{\star}$,

$$
\begin{gathered}
\left\langle u_{k}^{\star}, b-y_{k}\right\rangle=-\frac{\varphi(b)-\varphi(a)}{\|b-a\|}\left\|y_{k}-b\right\|, \quad\left\|u_{k}^{\star}\right\|=\frac{|\varphi(b)-\varphi(a)|}{\|b-a\|}, \\
\left\langle v_{k}^{\star}, x-y_{k}\right\rangle \leq 0 \quad \forall x \in[a, b],
\end{gathered}
$$

and

$$
x_{k}^{\star}+u_{k}^{\star}+v_{k}^{\star}+w_{k}^{\star}=0 \quad \forall k=1,2, \ldots
$$

It follows from (8.6), (8.8), and (8.7) as $x=b$ that

$$
\left\langle x_{k}^{\star}, b-y_{k}\right\rangle \geq \frac{\varphi(b)-\varphi(a)}{\|b-a\|}\left\|y_{k}-b\right\|-\frac{1}{k}\left\|y_{k}-b\right\| \forall k=1,2, \ldots .
$$

Let us observe that

$$
(x-b)\|y-b\|=(y-b)\|x-b\| \forall x, y \in[a, b] .
$$

So multiplying both side of (8.9) by $\left\|x_{k}-b\right\| / y_{k}-b \|$, using (8.10) for $x=x_{k}, y=y_{k}$, and taking the liminf as $k \rightarrow \infty$, we obtain (8.2). Similarly, (8.9) and (8.10) with $x=a, y=y_{k}$ yield (8.3).

Now let $c \neq a$. Without loss of generality we assume that $y_{k} \neq a$ for $k=1,2, \ldots$ In this case one easily gets from (8.7) the equality $\left\langle v_{k}^{\star}, b-c\right\rangle=0$ for all $k$. Using this equality together with (8.6) and (8.8), we have

$$
\left\langle x_{k}^{\star}, b-c\right\rangle=\frac{\varphi(b)-\varphi(a)}{\|b-a\|}\left\|y_{k}-b\right\|+\left\langle u_{k}^{\star}, c-y_{k}\right\rangle-\left\langle w_{k}^{\star}, b-c\right\rangle
$$

for all $k=1,2, \ldots$ Noting that $\left\langle u_{k}^{\star}, c-y_{k}\right\rangle \rightarrow 0$ and $\left\langle w_{k}^{\star}, b-c\right\rangle \rightarrow 0$ as $k \rightarrow \infty$, we obtain from (8.11) the equality

$$
\lim _{k \rightarrow \infty}\left\langle x_{k}^{\star}, b-c\right\rangle=\frac{\varphi(b)-\varphi(a)}{\|b-a\|}\|c-b\|,
$$

that yields (8.4) by virtue of (8.10) for $x=a$ and $y=c$. This completes the proof of the theorem.

8.3. Remark. Using another procedure, Zagrodny [70] first proved his approximate mean value theorem for the Clarke subdifferential in Banach spaces. In fact his proof automatically works for any subdifferentials satisfying certain requirements mentioned in [14]. Due to the sum rule in Theorem 4.1, those requirements hold for our basic subdifferential (2.7) in Asplund spaces, but not for its Fréchet counterpart 
$\hat{\partial} \varphi$. Loewen [40] established a version of the approximate mean value theorem for the Fréchet subdifferential in a Banach space whose norm is Fréchet smooth outside the origin. To prove this result, he employed a "fuzzy sum rule" for Fréchet subgradients and some properties of the smooth norm. After completing this paper, we received Thibault's preprint [66] that contains a proof of a "presubdifferential" version of the approximate mean value theorem based on the main idea of Zagrodny [70] and also some ideas of Borwein and Preiss [6]. Using Proposition 2.7 or the sum rule (4.2) proved above, we can conclude that the Fréchet subdifferential in Asplund spaces is a presubdifferential in the sense of [66] and, therefore, conditions (8.2) and (8.3) in Theorem 8.2 follow from the corresponding results of [66]. Note that our proof of the approximate mean value theorem is different from those in $[40,66,70]$.

It is worth mentioning that the mean value inequality (8.3) holds even in the case when $\varphi(b)=\infty$. Indeed, one has the following easy consequence of Theorem 8.2 .

8.4. Corollary. Let $X$ be an Asplund space and let $\varphi: X \rightarrow(-\infty, \infty]$ be a l.s.c. function finite at some point $a \in X$. Then for any $b \in X$ there exist $c \in[a, b]$ and $a$ pair of sequences $x_{k} \rightarrow c$ as $k \rightarrow \infty$ and $x_{k}^{\star} \in \hat{\partial} \varphi\left(x_{k}\right), k=1,2, \ldots$, such that (8.3) holds.

Proof. It remains to prove (8.3) when $\varphi(b)=\infty$. To do this we consider, following $[40,70]$, a sequence of functions

$$
\phi_{k}(x)= \begin{cases}\varphi(x) & \text { if } x \neq b \\ \varphi(a)+k & \text { if } x=b\end{cases}
$$

and employ Theorem 8.2 for each $k=1,2, \ldots$

8.5. Corollary. Under the assumptions of Corollary 8.4, for any $b \in X$ and $\varepsilon>0$ one has

$$
|\varphi(b)-\varphi(a)| \leq\|b-a\| \sup \left\{\left\|x^{\star}\right\| \text { s.t. } x^{\star} \in \hat{\partial} \varphi(c), c \in[a, b]+\varepsilon B\right\} .
$$

Proof. This follows directly from Corollary 8.4.

When $\varphi$ is Lipschitz continuous, we can pass to the limit in (8.3) and obtain a mean value inequality in terms of the subdifferential (2.7).

8.6. Corollary. Let $X$ be an Asplund space, let $U \in X$ be an open set containing $[a, b]$, and let $\varphi: U \rightarrow \mathbf{R}$ be Lipschitz continuous. Then one has

$$
\left\langle x^{\star}, b-a\right\rangle \geq \varphi(b)-\varphi(a) \text { for some } x^{\star} \in \partial \varphi(c), c \in[a, b) .
$$

Proof. According to Theorem 8.2 one can find a point $c \in[a, b)$ and sequences $x_{k} \rightarrow$ $c, x_{k}^{\star} \in \hat{\partial} \varphi\left(x_{k}\right)$ satisfying (8.3). It follows directly from the Lipschitz continuity of $\varphi$ and representation (2.11) as $\varepsilon=0$ that the sequence $\left\{x_{k}^{\star}\right\}$ is bounded in $X^{\star}$ and, due to Asplundity of $X$, is weak-star sequentially compact. Denoting by $x^{\star}$ its limiting point, one has $x^{\star} \in \partial \varphi(c)$ due to (2.9). This proves (8.12).

8.7. Remark. Using Corollary 8.6 and arguments similar to Borwein and Preiss [6, Theorem 3.2], one can establish the mean value inequality (8.12) with $c \in(a, b)$. It was proved in [6] for spaces with smooth renorms. 
Now let us consider some useful applications of the approximate mean value theorem in Asplund spaces. The first one characterizes the local Lipschitzian behavior of l.s.c. functions, generalizing Loewen's results in [40, Theorem 4.1].

8.8. Theorem. Let $X$ be an Asplund space and let $\varphi: X \rightarrow(-\infty, \infty]$ be a l.s.c. function finite at $\bar{x}$. Then the following assertions involving a constant $l \geq 0$ are equivalent:

(a) There exists $\gamma>0$ such that

$$
\hat{\partial} \varphi(x) \subset l B^{\star} \text { for any } x \in B_{\gamma}(\bar{x}) \text { with }|\varphi(x)-\varphi(\bar{x})| \leq \gamma .
$$

(b) $\hat{\partial} \varphi(x) \subset l B^{\star}$ in some neighborhood of $\bar{x}$.

(c) $\varphi$ is Lipschitz continuous around $\bar{x}$ with modulus $l$.

Proof. To prove $(\mathrm{a}) \Longrightarrow$ (b) we use Corollary 8.4 and arguments similar to [40, Theorem 4.1]. Implication (b) $\Longrightarrow$ (c) follows from Corollary 8.5. The Lipschitz condition in (c) implies (a) by virtue of (2.11) as $\varepsilon=0$.

8.9. Corollary. Let $X$ be an Asplund space, let $\varphi: X \rightarrow(-\infty, \infty]$ be a l.s.c. function, and let $U \subset X$ be an open set. Then $\varphi$ is locally constant in $U$ if and only if

$$
x^{\star} \in \hat{\partial} \varphi(x) \Longrightarrow x^{\star}=0 \forall x \in U .
$$

Thus (8.13) is equivalent to $\varphi$ being constant on $U$ if $U$ is connected.

Proof. This follows from Theorem 8.8 as $l=0$.

8.10. Remarks. (a) The result obtained implies a nonconvex generalization of the Bishop-Phelps density theorem in Corollary 3.4. Indeed, we take $\varphi(x)=\delta(x, \Omega)$ and use (2.13) as $\varepsilon=0$.

(b) An analogue of Corollary 8.9 for the proximal subdifferential was first obtained by Clarke [12] in finite dimensions and then was generalized by Clarke, Stern, and Wolenski [13] to the case of Hilbert spaces. Moreover, the paper [13] contains a proximal analogue of Theorem 8.8 and its application to monotonicity properties of l.s.c. functions in Hilbert spaces. The latter result in terms of Fréchet subgradients was obtained by Loewen [40] on the basis of the approximate mean value theorem in spaces with Fréchet differentiable norms. Developing this line and applying Corollary 8.4, we can easily get the monotonicity result in the Asplund space setting.

To conclude this section we use the results obtained above to establish relationships between our sequential limiting constructions and Clarke's concepts of generalized normals and subgradients. According to [10], Clarke's normal cone and generalized gradient (subdifferential) for extended-real-valued functions in Banach spaces are defined by the following three-step procedure. If $\varphi: X \rightarrow \mathbf{R}$ is Lipschitz continuous around $\bar{x}$, then

$$
\partial_{C} \varphi(\bar{x}):=\left\{x^{\star} \in X^{\star} \mid\left\langle x^{\star}, v\right\rangle \leq \varphi^{\circ}(\bar{x} ; v) \quad \forall v \in X\right\},
$$

where the generalized directional derivative is given by

$$
\varphi^{\circ}(\bar{x} ; v):=\limsup _{x \rightarrow \bar{x}, t \downarrow 0}[\varphi(x+t v)-\varphi(x)] / t .
$$


Further, Clarke's normal cone can be defined by using the generalized gradient (8.14) of the Lipschitz continuous distance function

$$
N_{C}(\bar{x} ; \Omega):=\operatorname{cl}^{\star}\left[\bigcup_{\lambda>0} \lambda \partial_{C} \operatorname{dist}(\bar{x}, \Omega)\right] \forall \bar{x} \in \operatorname{cl} \Omega
$$

Finally, if $\varphi: X \rightarrow \overline{\mathbf{R}}$ is finite at $\bar{x}$, then

$$
\partial_{C} \varphi(\bar{x}):=\left\{x^{\star} \in X^{\star} \mid\left(x^{\star},-1\right) \in N_{C}((\bar{x}, \varphi(\bar{x})) ; \text { epi } \varphi)\right\} .
$$

It is proved in [31] that in any Banach space $X$ one always has the inclusions

$$
N(\bar{x} ; \Omega) \subset N_{C}(\bar{x} ; \Omega) \text { and } \partial \varphi(\bar{x}) \subset \partial_{C} \varphi(\bar{x})
$$

for the normal cone (2.2) and the subdifferential (2.7). Let us obtain more precise relationships between the constructions under consideration in Asplund spaces.

8.11. Theorem. Let $X$ be an Asplund space. Then the following relationships hold:

(i) For any closed set $\Omega \subset X$ and $\bar{x} \in \Omega$ one has

$$
N_{C}(\bar{x} ; \Omega)=\operatorname{cl}^{\star} \operatorname{co} N(\bar{x} ; \Omega) .
$$

(ii) For any function $\varphi: X \rightarrow \overline{\mathbf{R}}$ l.s.c. around $\bar{x} \in \operatorname{dom} \varphi$ one has

$$
\partial_{C} \varphi(\bar{x})=\operatorname{cl}^{\star} \operatorname{co}\left[\partial \varphi(\bar{x})+\partial^{\infty} \varphi(\bar{x})\right] .
$$

If, in particular, $\varphi$ is Lipschitz continuous around $\bar{x}$, then

$$
\partial_{C} \varphi(\bar{x})=\operatorname{cl}^{\star} \operatorname{co} \partial \varphi(\bar{x})
$$

Proof. First let us consider the case when $\varphi$ is Lipschitz continuous in some neighborhood $U$ of $\bar{x}$. Using condition (8.3) in Theorem 8.2 and the construction of Clarke's directional derivative (8.15), we can establish the representation

$$
\varphi^{\circ}(\bar{x} ; v)=\max \left\{\left\langle x^{\star}, v\right\rangle \mid x^{\star} \in \partial \varphi(\bar{x})\right\},
$$

that holds for any $v \in X$; cf. [6, proof of Theorem 3.3]. The latter implies (8.21) by virtue of (8.14). Applying (8.21) to the distance function $\varphi(x)=\operatorname{dist}(x, \Omega)$, one has

$$
\bigcup_{\lambda>0} \lambda \partial_{C} \operatorname{dist}(x ; \Omega)=\bigcup_{\lambda>0} \lambda\left[\operatorname{cl}^{\star} \operatorname{co} \partial \operatorname{dist}(x, \Omega)\right] \subset \operatorname{cl}^{\star} \operatorname{co}\left[\bigcup_{\lambda>0} \lambda \partial \operatorname{dist}(x, \Omega)\right] .
$$

This yields $N_{C}(\bar{x} ; \Omega) \subset \mathrm{cl}^{\star} \operatorname{co} N(\bar{x} ; \Omega)$ by virtue of (8.16) and Proposition 2.6. The opposite inclusion in (8.19) follows directly from the first part of (8.18) and the fact that Clarke's normal cone is convex and closed in the weak-star topology of $X^{\star}$.

It remains to prove (8.20) for any l.s.c. function $\varphi$ finite at $\bar{x}$. According to (2.7) and (2.8) one has $N((\bar{x}, \varphi(\bar{x}))$; epi $\varphi)=N_{1} \cup N_{2}$, where

$$
N_{1}:=\left\{\lambda\left(x^{\star},-1\right) \mid x^{\star} \in \partial \varphi(\bar{x}), \lambda>0\right\} \text { and } N_{2}:=\left\{\left(x^{\star}, 0\right) \mid x^{\star} \in \partial^{\infty} \varphi(\bar{x})\right\} .
$$

Thus (8.20) follows from (2.17) and (2.19) due to Loewen's extension [37] of the Rockafellar finite dimensional result [59]. (Note that Proposition 4.2 in [37] is presented for normed linear spaces but its proof is actually valid for any locally convex spaces, i.e., it covers the case of $X^{\star}$ ). This completes the proof of the theorem. 
8.12. Remarks. (a) In finite dimensions, the basic relationship (8.19) is equivalent to the Clarke proximal normal formula; cf. [10, 39, 63]. In [67], Treiman obtained the first infinite dimensional version of (8.19) and the associated subdifferential formulas in spaces with Fréchet differentiable renorms using the original definition (2.2) with $\varepsilon \downarrow 0$. Borwein and Strojwas [8] showed that one can let $\varepsilon=0$ in those formulas if $X$ is reflexive; cf. also an alternative proof in Loewen [37] for the case of Hilbert spaces. Borwein and Preiss [6] established formula (8.21) for locally Lipschitz functions in spaces with Fréchet differentiable renorms, while the other relationships (8.19) and (8.20) in the same setting were first mentioned by Ioffe [25]. Theorem 8.11 contains extensions of these results to the case of Asplund spaces.

(b) In [57], Preiss obtained a profound refinement of formula (8.21) for locally Lipschitz functions in Asplund spaces. In the refined formula, the sequential limiting points of Fréchet subgradients in (8.21) are replaced by those for the classical Fréchet derivative of $\varphi$, which is proved to exist on a dense set.

\section{Connections With approximate subdifferentials}

In this section we study relationships between our sequential normal and subdifferential constructions and the "approximate subdifferentials" of Ioffe [22, 23, 24], who developed another line of infinite dimensional generalizations of the nonconvex constructions in [41, 42]. Ioffe proposed several modifications of approximate subdifferentials; some of them are well defined in arbitrary locally convex spaces. Loosely speaking, all these subdifferentials are related to upper limits of more primitive subdifferentials based on the Dini directional derivatives. Here we consider the case of Asplund spaces, where these constructions can be defined as follows. (Note that any Asplund space is a "weakly trustworthy" space; cf. [23, 18]).

Let $\varphi: X \rightarrow \overline{\mathbf{R}}$ be 1.s.c. around $\bar{x} \in \operatorname{dom} \varphi$. First the $A$-subdifferential of $\varphi$ at $\bar{x}$ is defined by taking the topological upper limit

$$
\partial_{A} \varphi(\bar{x}):=\operatorname{Limsup}_{x \stackrel{\varphi}{\rightarrow} \bar{x}, \varepsilon \downarrow 0} \partial_{\varepsilon}^{-} \varphi(x)
$$

(with respect to the norm topology in $X$ and the weak-star topology in $X^{\star}$ ) of the Dini subdifferential constructions

$$
\partial_{\varepsilon}^{-} \varphi(x):=\left\{x^{\star} \in X^{\star} \mid\left\langle x^{\star}, v\right\rangle \leq d \varphi(x ; v)+\varepsilon\|v\| \forall v \in X\right\},
$$

where

$$
d \varphi(x ; v):=\liminf _{u \rightarrow v, t \downarrow 0} t^{-1}(\varphi(x+t u)-\varphi(x)) .
$$

Then, using the $A$-subdifferential of the Lipschitz continuous distance function, the $G$-normal cone to $\Omega \subset X$ at $\bar{x} \in \mathrm{cl} \Omega$ is defined by

$$
N_{G}(\bar{x} ; \Omega):=\operatorname{cl}^{\star} \check{N}_{G}(\bar{x} ; \Omega),
$$

where the construction

$$
\check{N}_{G}(\bar{x} ; \Omega):=\bigcup_{\lambda>0} \lambda \partial_{A} \operatorname{dist}(\bar{x}, \Omega)
$$

is called the nucleus of $N_{G}(\bar{x} ; \Omega)$. Finally, the $G$-subdifferential of $\varphi$ at $\bar{x}$ is defined geometrically as

$$
\partial_{G} \varphi(\bar{x}):=\left\{x^{\star} \in X^{\star} \mid\left(x^{\star},-1\right) \in N_{G}((\bar{x}, \varphi(\bar{x})) ; \text { epi } \varphi)\right\},
$$


while its $G$-nucleus $\check{\partial ̆}_{G} \varphi(\bar{x})$ corresponds to $(9.4)$ with $N_{G}$ replaced by $\check{N}_{G}$. Note that constructions (9.1) and (9.3) coincide with those introduced in [20] under the name of $M$-subdifferential and $M$-normal cone, respectively.

Ioffe proves in [24] that the $G$-subdifferential (9.4) is always smaller than its $A$-counterpart (9.1), and they both coincide with the $G$-nucleus $\check{\partial}_{G} \varphi(\bar{x})$ for directionally Lipschitz functions. Using the sequential (vs. topological) upper limit in (9.1), one can consider the sequential analogues

$$
\begin{gathered}
\partial_{A}^{\sigma} \varphi(\bar{x}):=\lim _{\substack{\varphi \\
x \rightarrow \bar{x}, \varepsilon \downarrow 0}} \partial_{\varepsilon}^{-} \varphi(x), \quad \check{N}_{G}^{\sigma}(\bar{x} ; \Omega):=\bigcup_{\lambda 0} \lambda \partial_{A}^{\sigma} \operatorname{dist}(\bar{x}, \Omega), \\
\check{\partial}_{G}^{\sigma} \varphi(\bar{x}):=\left\{x^{\star} \in X^{\star} \mid\left(x^{\star},-1\right) \in \check{N}_{G}^{\sigma}((\bar{x}, \varphi(\bar{x})) ; \operatorname{epi} \varphi)\right\}
\end{gathered}
$$

of the topological objects defined above; cf. [25]. The question is: how do all these constructions relate to our sequential normal cone (2.2) and subdifferential (2.7)? To answer this question we will use some recent results of Borwein and Fitzpatrick [4].

Recall that a Banach space $X$ is weakly compactly generated (WCG) provided there is a weakly compact set $K$ such that $X=\operatorname{cl}($ span $K)$. Clearly all reflexive Banach spaces and all separable Banach spaces are weakly compactly generated. For the case of Asplund spaces, there are precise characterizations of the WCG property; see $[4,16]$. Note that the WCG property subtantially narrows the class of Asplund spaces; it implies, in particular, the existence of a Fréchet differentiable renorm [16]. Let us present the basic interrelations between weak-star topological and sequential limits that were proved in [4, Theorem 1.3].

9.1. Proposition. Let $X$ be a Banach space and let $\left\{S_{k}\right\}$ be a sequence of bounded subsets of $X^{\star}$ such that $S_{k+1} \subset S_{k}$ for all $k=1,2, \ldots$ The following assertions hold:

(i) If the unit ball of $X^{\star}$ is weak-star sequentially compact, then

$$
\bigcap_{k=1}^{\infty} \operatorname{cl}^{\star} S_{k}=\operatorname{cl}^{\star}\left\{\lim _{k \rightarrow \infty} x_{k}^{\star} \mid x_{k}^{\star} \in S_{k} \text { for all } k\right\} .
$$

(ii) If $X$ is a subspace of a WCG space, then

$$
\bigcap_{k=1}^{\infty} \operatorname{cl}^{\star} S_{k}=\left\{\lim _{k \rightarrow \infty} x_{k}^{\star} \mid x_{k}^{\star} \in S_{k} \text { for all } n\right\} .
$$

Now we establish the main relationships between the subdifferential and normal cone constructions under consideration.

9.2. Theorem. Let $X$ be an Asplund space. Then the following hold:

(i) For any function $\varphi: X \rightarrow \bar{R}$ l.s.c. around $\bar{x} \in \operatorname{dom} \varphi$ one has the inclusions

$$
\partial \varphi(\bar{x}) \subset \partial_{A}^{\sigma} \varphi(\bar{x}) \subset \partial_{A} \varphi(\bar{x}) .
$$

Moreover, if $\varphi$ is Lipschitz continuous around $\bar{x}$, then

$$
\operatorname{cl}^{\star}(\partial \varphi(\bar{x}))=\operatorname{cl}^{\star}\left(\partial_{A}^{\sigma} \varphi(\bar{x})\right)=\partial_{A} \varphi(\bar{x}) .
$$

In the latter case the sets $\partial \varphi(\bar{x})$ and $\partial_{A}^{\sigma} \varphi(\bar{x})$ are weak-star closed, and

$$
\partial \varphi(\bar{x})=\partial_{A}^{\sigma} \varphi(\bar{x})=\partial_{A} \varphi(\bar{x})
$$

if $X$ is a WCG space. 
(ii) Let $\Omega \subset X$ and $\bar{x} \in \operatorname{cl} \Omega$. Then

$$
N(\bar{x} ; \Omega) \subset \check{N}_{G}^{\sigma}(\bar{x} ; \Omega) \subset \check{N}_{G}(\bar{x} ; \Omega) \subset N_{G}(\bar{x} ; \Omega)=\operatorname{cl}^{\star} N(\bar{x} ; \Omega) .
$$

Moreover, if $X$ is a WCG space, then

$$
N(\bar{x} ; \Omega)=\check{N}_{G}^{\sigma}(\bar{x} ; \Omega)=\check{N}_{G}(\bar{x} ; \Omega) .
$$

Proof. One can easily check from the definitions that $\hat{\partial} \varphi(x) \subset \partial_{\varepsilon}^{-} \varphi(x)$ for any $x \in \operatorname{dom} \varphi$ and $\varepsilon \geq 0$. Therefore, inclusions (9.6) follow directly from (9.1), (9.5), and Theorem 2.9(iii).

Let us prove (9.7) for any function $\varphi$ that is Lipschitz continuous around $\bar{x}$. Based on the definitions, we observe the following representations:

$$
\partial_{A} \varphi(\bar{x})=\bigcap_{k=1}^{\infty} \mathrm{cl}^{\star} S_{k} \text { and } \partial_{A}^{\sigma} \varphi(\bar{x})=\left\{\lim _{k \rightarrow \infty} x_{k}^{\star} \mid x_{k}^{\star} \in S_{k} \text { for all } k\right\},
$$

where $S_{k}:=\bigcup\left\{\partial_{1 / k}^{-} \varphi(x)\right.$ with $\left.\|x-\bar{x}\| \leq 1 / k\right\}$. Obviously $S_{k+1} \subset S_{k}$ for all $k=1,2, \ldots$ Moreover, all the sets $S_{k}$ are bounded in $X^{\star}$ due to the Lipschitz continuity of $\varphi$ around $\bar{x}$. Employing Proposition 9.1(i), we conclude that $\partial_{A} \varphi(\bar{x})=$ $\mathrm{cl}^{\star}\left(\partial_{A}^{\sigma} \varphi(\bar{x})\right)$. To establish (9.7) it remains to verify $\partial_{A}^{\sigma} \varphi(\bar{x}) \subset \operatorname{cl}^{\star}(\partial \varphi(\bar{x}))$. The latter follows from the inclusion

$$
\partial_{A}^{\sigma} \varphi(\bar{x}) \subset \partial \varphi(\bar{x})+V^{\star}
$$

for any weak-star neighborhood $V^{\star}$ of the origin in $X^{\star}$.

To prove (9.12) we observe that for any neighborhood $V^{\star}$ under consideration there exist a finite dimensional subspace $L \subset X$ and a number $r>0$ such that

$$
L^{0}+3 r B^{\star} \subset V^{\star}, \quad \text { where } L^{0}:=\left\{x^{\star} \in X^{\star} \mid\left\langle x^{\star}, x\right\rangle \leq 0 \quad \forall x \in L\right\} .
$$

Let $x^{\star} \in \partial_{A}^{\sigma} \varphi(\bar{x})$, i.e., there exist $x_{k} \rightarrow \bar{x}, \varepsilon_{k} \downarrow 0$, and $x_{k}^{\star} \stackrel{w^{\star}}{\rightarrow} x^{\star}$ with $x_{k}^{\star} \in \partial_{\varepsilon_{k}}^{-} \varphi\left(x_{k}\right)$ for all $k$. We always take $k$ big enough to get $0<\varepsilon_{k} \leq r$ and $1 / k \leq r$. Using the definition of the Dini $\varepsilon$-subdifferential, one concludes that for any $r>0$ and finite dimensional subspace $L \subset X$ the function

$$
f_{k}(x):=\varphi(x)-\left\langle x_{k}^{\star}, x-x_{k}\right\rangle+2 r\left\|x-x_{k}\right\|+\delta\left(x-x_{k}, L\right)
$$

attains a local minimun at $x_{k}$ for each $k$; cf. [22, Lemma 1]. Therefore, $0 \in \hat{\partial} f_{k}\left(x_{k}\right)$ and we can apply Proposition 2.7 for the sum of functions in (9.13). Employing this result with $\varepsilon=0$ and $\gamma=r$, we find $\tilde{x}_{k} \in B_{1 / k}\left(x_{k}\right)$ such that

$$
x_{k}^{\star} \in \hat{\partial} \varphi\left(\tilde{x}_{k}\right)+3 r B^{\star}+L^{0} \subset \hat{\partial} \varphi\left(\tilde{x}_{k}\right)+V^{\star}
$$

for all $k$. Since $\varphi$ is Lipschitz continuous around $\bar{x}$, one has the uniform boundedness of $\hat{\partial} \varphi(x)$ around this point due to Theorem 8.8. Taking into account the weak-star sequential compactness of bounded sets in $X^{\star}$ and representation (2.9), we obtain (9.12) by passing to the limit in (9.14) as $k \rightarrow \infty$. This proves equalities (9.7).

Now let $X$ be a WCG space, while $\varphi$ is still Lipschitz continuous around $\bar{x}$. Then one has $\operatorname{cl}^{\star}\left(\partial_{A}^{\sigma} \varphi(\bar{x})\right)=\partial_{A}^{\sigma} \varphi(\bar{x})=\partial_{A} \varphi(\bar{x})$ due to (9.11) and Proposition 9.1(ii). Using the same procedure, we conclude that $\partial \varphi(\bar{x})$ is weak-star closed; cf. [4]. Therefore, (9.8) follows from (9.7) in the WCG case.

To establish the normal cone relationships (9.9) and (9.10) in (ii) we just observe that all these conclusions follow from (i) and Proposition 2.6 due to definitions (9.2), (9.3), and (9.5). This completes the proof of the theorem. 
9.3. Corollary. Let $X$ be an Asplund space and let $\varphi: X \rightarrow \overline{\mathbf{R}}$ be l.s.c. around $\bar{x}$. Then one has

$$
\partial \varphi(\bar{x}) \subset \check{\partial}_{G}^{\sigma} \varphi(\bar{x}) \subset \check{\partial}_{G} \varphi(\bar{x}) \subset \partial_{G} \varphi(\bar{x})=\operatorname{cl}^{\star}(\partial \varphi(\bar{x})) .
$$

If, in addition, $X$ is a WCG space and $\varphi$ is Lipschitz continuous around $\bar{x}$, then

$$
\partial \varphi(\bar{x})=\check{\partial}_{G}^{\sigma} \varphi(\bar{x})=\check{\partial}_{G} \varphi(\bar{x})=\partial_{G} \varphi(\bar{x}) .
$$

Proof. The inclusions in (9.15) immediately follow from (9.9) by virtue of the definitions. To obtain the equality in (9.15) from the one in (9.9) we observe that

$L \cap N_{G}((\bar{x}, \varphi(\bar{x})) ; \operatorname{epi} \varphi)=L \cap \operatorname{cl}^{\star} N((\bar{x}, \varphi(\bar{x})) ; \operatorname{epi} \varphi)=\operatorname{cl}^{\star}(L \cap N((\bar{x}, \varphi(\bar{x}))$; epi $\varphi))$ with $L:=X^{\star} \times\{-1\} \subset X^{\star} \times \mathbf{R}$. Finally, under the WCG property of $X$ and the Lipschitz continuity of $\varphi$ the set $\partial \varphi(\bar{x})$ is weak-star closed (see the proof of Theorem 9.2) and equalities (9.16) follow from (9.15). This proves the corollary.

9.4. Remarks. (a) Theorem 9.2 immediately implies the same relationships (9.5) between the singular subdifferential (2.8) and its $G$-counterparts for any l.s.c. functions in Asplund spaces.

(b) Equalities (9.16) can be established not only for locally Lipschitz functions but also for a more general class of functions satisfying the normal compactness condition (Section 4) in WCG Asplund spaces. This follows from the fact that $\partial \varphi(\bar{x})$ is weak-star closed for such functions. The latter is proved by Loewen [38] for reflexive spaces (that is essential in his proof) and appears in our paper [53] in the general case considered. Similarly, all the normal cones in (9.10) are weak-star closed and coincide with $N_{G}(\bar{x} ; \Omega)$ if $\Omega$ is normally compact around $\bar{x}$ in the case of WCG Asplund spaces.

(c) In [4], Borwein and Fitzpatrick give examples showing that sequential and topological limits of derivative objects can provide quite different sets outside of WCG spaces. In particular, their Example 2.4 demonstrates that our basic sequential subdifferential (2.7) may be strictly smaller than the $G$-subdifferential (9.4) and its nucleus even for the case of Lipschitz continuous (concave) functions defined on spaces with Fréchet differentiable norms.

(d) In [25], Ioffe proves the equality $N_{G}(\bar{x} ; \Omega)=\operatorname{cl}^{\star} N(\bar{x} ; \Omega)$ and the corresponding equalities for the subdifferentials in the case of spaces with Fréchet differentiable renorms. Note that Theorem 1 in [25] actually claims the equality $N(\bar{x} ; \Omega)=\check{N}_{G}^{\sigma}(\bar{x} ; \Omega)$, but there is a $g a p$ in the proof of the basic Lemma 4 therein related to the difference between the sequential and topological closures.

In [5], Borwein and Ioffe establish that the nucleus (8.3) of the $G$-normal cone and the corresponding subdifferential can be represented as weak-star closures of sequential constructions in Banach spaces having differentiable renorms of any kind (Fréchet, Gâteaux, etc.). For the case of spaces with Fréchet differentiable renorms they prove the representation

$$
\check{N}_{G}(\bar{x} ; \Omega)=\bigcup_{l=1}^{\infty} \operatorname{cl}^{\star}\left\{\lim _{k \rightarrow \infty} x_{k}^{\star} \mid x_{k}^{\star} \in N_{F}^{l}\left(x_{k} ; \Omega\right), x_{k} \stackrel{\Omega}{\rightarrow} \bar{x}\right\},
$$

where $N_{F}^{l}(x ; \Omega)$ is the collection of all F-normals of rank $l$ to the closed set $\Omega$ at $x \in \Omega$, defined as follows: $x^{\star} \in N_{F}^{l}(x ; \Omega)$ if there exists a function $\psi: X \rightarrow \mathbf{R}$ Fréchet differentiable in a neighborhood $U$ of $x$ such that $x^{\star}=\psi^{\prime}(x), \psi(x)=0, \psi$ is Lipschitz continuous on $U$ with modulus $l$, and $\psi(u) \leq 0$ for all $u \in \Omega \cap U$. 
Let us show that our sequential normal cone (2.2) is related to the limiting set in (9.17) with no weak-star closure.

9.5. Theorem. Let $X$ be a Banach space having an equivalent Fréchet differentiable norm and let $\Omega$ be a closed subset of $X$. Then for any $\bar{x} \in \Omega$ one has

$$
N(\bar{x} ; \Omega)=\bigcup_{l=1}^{\infty}\left\{\lim _{k \rightarrow \infty} x_{k}^{\star} \mid x_{k}^{\star} \in N_{F}^{l}\left(x_{k} ; \Omega\right), x_{k} \stackrel{\Omega}{\rightarrow} \bar{x}\right\} .
$$

Proof. It easily follows from the definitions that the right-hand side of (9.18) cannot be bigger than $N(\bar{x} ; \Omega)$. Let us prove the opposite inclusion using the normal cone representation in Proposition 2.6. To this end we pick any $x^{\star} \in \partial \operatorname{dist}(\bar{x}, \Omega)$ and then employ Theorem 2.9(iii) to find sequences $x_{k} \rightarrow \bar{x}$ and $x_{k}^{\star} \stackrel{w^{\star}}{\rightarrow} x^{\star}$ as $k \rightarrow \infty$ with $x_{k}^{\star} \in \hat{\partial} \operatorname{dist}\left(x_{k}, \Omega\right)$ for all $k$. Following the proof of Proposition 1 in [5], one can establish that for any $u^{\star} \in \hat{\partial} \operatorname{dist}(u, \Omega), \varepsilon>0$, and $l>1$ there exist $v \in \Omega$ and $v^{\star} \in N_{F}^{l}(v ; \Omega)$ such that $\|v-u\| \leq \operatorname{dist}(u, \Omega)+\varepsilon$ and $v^{\star} \in u^{\star}+\varepsilon B^{\star}$. Now taking $\left(u, u^{\star}\right)=\left(x_{k}, x_{k}^{\star}\right)$ and a sequence $\varepsilon_{k} \downarrow 0$ as $k \rightarrow \infty$, for any natural numbers $k$ and $l$ we find $\tilde{x}_{k} \in \Omega$ and $\tilde{x}_{k}^{\star} \in N_{F}^{l}\left(\tilde{x}_{k} ; \Omega\right)$ (drop the index $l$ ) such that $\left\|\tilde{x}_{k}-x_{k}\right\| \leq\left\|x_{k}-\bar{x}\right\|+\varepsilon_{k}$ and $\left\|\tilde{x}_{k}^{\star}-x_{k}^{\star}\right\| \leq \varepsilon_{k}$. Thus $\tilde{x}_{k} \rightarrow \bar{x}$ and $\tilde{x}_{k}^{\star} \rightarrow x^{\star}$ as $k \rightarrow \infty$. This completes the proof of the theorem.

9.6. Remark. Similarly we can establish representations of the subdifferential (2.7) and the singular subdiffefential (2.8) in terms of the limiting $F$-subdifferentials of rank $l[5]$ for spaces with Fréchet differentiable renorms.

As one can see, the sequential subdifferential (2.7) turns out to be the smallest among all other subdifferentials considered above. Let us show that (2.7) is actually minimal among any of the abstract subdifferentials satisfying natural requirements.

9.7. Theorem. Let $X$ be a Banach space and let $D \varphi: X \Rightarrow X^{\star}$ be an arbitrary subdifferential mapping on the class of functions $\varphi: X \rightarrow \overline{\mathbf{R}}$ with the invariance property

$$
D \phi(u)=D \varphi(x+u) \text { for } \phi(u)=\varphi(x+u) \quad \forall x, u \in X .
$$

Assume that the subdifferential D $\varphi$ satisfies the following two requirements:

(i) $D \varphi(u)$ is contained in the subdifferential of convex analysis for convex functions of the form

$$
\varphi(u)=\left\langle u^{\star}, u\right\rangle+\alpha\|u\| \forall u^{\star} \in X^{\star}, \alpha>0 .
$$

(ii) For any functions $\varphi_{i}, i=1,2$, such that $\varphi_{1}$ is l.s.c. around $u=0, \varphi_{2}$ is convex of form (9.20), and the sum $\varphi_{1}+\varphi_{2}$ attains a local minimum at $u=0$, one has

$$
0 \in \bigcap_{\gamma>0} \bigcup_{u_{i} \in U_{i \gamma}}\left\{D \varphi_{1}\left(u_{1}\right)+D \varphi_{2}\left(u_{2}\right)+\gamma B^{\star}\right\},
$$

where $U_{i \gamma}:=\left\{u \in X\right.$ with $\left.\|u\| \leq \gamma,\left|\varphi_{i}(u)-\varphi_{i}(0)\right| \leq \gamma\right\}, i=1,2$.

Then for any function $\varphi$ and any point $\bar{x} \in \operatorname{dom} \varphi$ around which $\varphi$ is l.s.c., one has the inclusion

$$
\partial \varphi(\bar{x}) \subset \limsup _{x \stackrel{\varphi}{\rightarrow} \bar{x}} D \varphi(x) .
$$


In particular, if $D \varphi$ is sequentially upper semicontinuous at $\bar{x}$ in the sense that

$$
D \varphi(\bar{x})=\limsup _{x \rightarrow \bar{\varphi}} D \varphi(x),
$$

then $\partial \varphi(\bar{x}) \subset D \varphi(\bar{x})$.

Proof. Let $\varphi$ be l.s.c. around $\bar{x}$ and let $x^{\star} \in \partial \varphi(\bar{x})$. By virtue of (2.12) one gets sequences $x_{k} \stackrel{\varphi}{\rightarrow} \bar{x}, x_{k}^{\star} \rightarrow x^{\star}$, and $\varepsilon_{k} \downarrow 0$ as $k \rightarrow \infty$ with $x_{k}^{\star} \in \tilde{\partial}_{\varepsilon_{k}} \varphi\left(x_{k}\right)$ for all $k$. Now using (2.11), we find a sequence $\delta_{k} \downarrow 0$ such that

$$
\varphi(x)-\varphi\left(x_{k}\right)-\left\langle x_{k}^{\star}, x-x_{k}\right\rangle \geq-2 \varepsilon_{k}\left\|x-x_{k}\right\| \forall x \in B_{\delta_{k}}\left(x_{k}\right), k=1,2, \ldots .
$$

The latter means that for any fixed $k$ the function

$$
f_{k}(u):=\varphi\left(x_{k}+u\right)-\left\langle x_{k}^{\star}, u\right\rangle+2 \varepsilon_{k}\|u\|
$$

attains a local minimum at $u=0$. Denoting $\varphi_{1}(u):=\varphi\left(x_{k}+u\right)$ and $\varphi_{2}(u):=$ $-\left\langle x_{k}^{\star}, u\right\rangle+2 \varepsilon_{k}\|u\|$, we represent (9.24) as the sum of two functions satisfying the assumptions in (ii). Now applying (9.21) along the sequence $\gamma=\varepsilon_{k}$ and then using properties (9.19) and (i), one gets $u_{k} \in X$ such that

$$
\begin{gathered}
\left\|u_{k}\right\| \leq \varepsilon_{k}, \quad\left|\varphi\left(x_{k}+u_{k}\right)-\varphi\left(x_{k}\right)\right| \leq \varepsilon_{k}, \text { and } \\
x_{k}^{\star} \in D \varphi\left(x_{k}+u_{k}\right)+3 \varepsilon_{k} B^{\star} \forall k=1,2, \ldots .
\end{gathered}
$$

Passing to the limit in (9.25) as $k \rightarrow \infty$, we obtain (9.22). The final inclusion $\partial \varphi(\bar{x}) \subset D \varphi(\bar{x})$ follows from (9.22) under the upper semicontinuity assumption (9.23). This ends the proof of the theorem.

9.8. Remarks. (a) The "zero fuzzy sum rule" (9.21) was first established by Ioffe [21] for Dini subdifferentials in finite dimensions. Such a rule appears to be necessary for any reasonable subdifferentials, and now it is known for most subdifferential constructions used in applications; see [5, 9, 11, 18, 25, 27, 40, 51] and their references. We observe that results in this vein are closely related to the $\varepsilon$-extremal principle in Section 3; see [51] for more discussions. Note that (9.21) immediately follows from the stationary principle and the "fuzzy sum rule" as in Proposition 2.7 with $\varepsilon=0$ for a subdifferential $D \varphi$. The latter obviously holds for any subdifferential satisfying the exact sum rule

$$
D\left(\varphi_{1}+\varphi_{2}\right)(\bar{x}) \subset D \varphi_{1}(\bar{x})+D \varphi_{2}(\bar{x})
$$

on the class of functions in requirement (ii) of Theorem 9.7. Thus our basic subdifferential (2.7) is the smallest among all reasonable subdifferential constructions subject to the sequential closure operation in (9.23). Note that the latter regularization procedure seems to be unavoidable for getting a full calculus and criteria for openness and metric regularity of nonsmooth mappings as well as for important applications to optimization, sensitivity, etc. According to the present paper and related developments in [53] we can conclude that such properties of the subdifferential (2.7) are available in the case of Asplund spaces.

(b) Theorem 9.7 generalizes the finite dimensional results in Ioffe [22] and Mordukhovich $[43,44]$, replacing the stationary and exact sum rule requirements by a more flexible requirement (9.21). In the case of Banach spaces, Ioffe [24, Theorem 8.1] proves that the $G$-nucleus (9.4) belongs to any subdifferential $D \varphi(\bar{x})$ which is topologically upper semicontinuous at $\bar{x}$ under some additional requirements. Note that Theorem 9.7 establishes the minimality of (2.7) in a more general class of subdifferentials, which may not be topologically closed. 
(c) In [24, Proposition 8.2], Ioffe states that $\check{\partial ̆}_{G} \varphi(\bar{x}) \subset \partial \varphi(\bar{x})$ for l.s.c. functions defined on spaces with Fréchet differentiable renorms. However, he proves in fact that the $G$-nucleus $\check{\partial}_{G} \varphi(\bar{x})$ is contained in the topological counterpart of (2.7), where the sequential upper limit in representation (2.12) is replaced by the topological one. The latter result follows directly from (9.15) in the general case of Asplund spaces. Moreover, in Corollary 9.3 we prove that just the opposite inclusion $\partial \varphi(\bar{x}) \subset \check{\partial}_{G} \varphi(\bar{x})$ holds for any l.s.c. function defined on an Asplund space. To this end we note that in general neither does $\check{\partial}_{G} \varphi$ admit the upper semicontinuity property topologically nor does $\partial \varphi$ have its sequential counterpart. Sufficient conditions for these properties can be found, respectively, in [24] and [38, 53].

\section{ACKNOWLEDGMENT}

We are indebted to J. M. Borwein, M. Fabian, L. Thibault, and the referee for helpful suggestions and remarks.

\section{REFERENCES}

1. E. Asplund, Fréchet differentiability of convex functions, Acta Math. 121 (1968), 31-47. MR 37:6754

2. J.-P. Aubin and H. Frankowska, Set-valued analysis, Birkhäuser, Boston, Mass., 1990. MR 91d:49001

3. J. M. Borwein, Epi-Lipschitz-like sets in Banach spaces: theorems and examples, Nonlinear Anal. 11 (1987), 1207-1217. MR 89b:90025

4. J. M. Borwein and S. P. Fitzpatrick, Weak-star sequential compactness and bornological limit derivatives, J. of Convex Analysis (to appear).

5. J. M. Borwein and A. D. Ioffe, Proximal analysis in smooth spaces, Set-Valued Anal. (to appear).

6. J. M. Borwein and D. Preiss, A smooth variational principle with applications to subdifferentiability and differentiability of convex functions, Trans. Amer. Math. Soc. 303 (1987), 517-527. MR 88k:49013

7. J. M. Borwein and H. M. Strojwas, Tangential approximations, Nonlinear Anal. 9 (1985), 1347-1366. MR 87i:90320

8. J. M. Borwein and H. M. Strojwas, Proximal analysis and boundaries of closed sets in Banach space, Part I: Theory, Can. J. Math. 38 (1986),431-452; Part II: Applications, Can. J. Math. 39(1987), 428-472. MR 87h:90258; MR 88f:46034

9. J. M. Borwein and Q. J. Zhu, Viscosity solutions and viscosity subderivatives in smooth Banach spaces with applications to metric regularity, SIAM J. Control Optim. (to appear).

10. F. H. Clarke, Optimization and nonsmooth analysis, Wiley, 1983. MR 85m:49002

11. F. H. Clarke, Methods of dynamic and nonsmooth optimization, CBMS-NSF Regional Conference Series in Applied Mathematics, vol. 57, Soc. Indust. Appl. Math., Philadelphia, Pa., 1989. MR 91j: 49001

12. F. H. Clarke, An indirect method in the calculus of variations, Trans. Amer. Math. Soc. 336 (1993), 655-573. MR 93f:49002

13. F. H. Clarke, R. J. Stern, and P. R. Wolenski, Subgradient criteria for monotonicity and the Lipschitz condition, Can. J. Math. 45 (1993), 1167-1183. MR 94j:49018

14. R. Correa, A. Jofré, and L. Thibault, Subdifferential monotonicity as characterization of convex functions, Numer. Funct. Anal. Optimiz. 15 (1994), 531-535. MR 95d:49029

15. M. G. Crandall and J. P. Lions, Hamilton-Jacobi equations in infinite dimensions. I: Uniqueness of viscosity solutions, J. Funct. Anal. 62 (1985), 379-396. MR 86j:35154

16. R. Deville, G. Godefroy, and V. Zizler, Smoothness and renorming in Banach spaces, Pitman Monographs and Surveys in Pure and Applied Math., vol. 64, Longman, 1993. MR 94d:46012

17. I. Ekeland, On the variational principle, J. Math. Anal. Appl. 47 (1974), 325-358. MR 49:11344

18. M. Fabian, Subdifferentiallity and trustworthiness in the light of a new variational principle of Borwein and Preiss, Acta Univ. Carolinae 30 (1989), 51-56. MR 91c:49024 
19. B. E. Ginsburg and A. D. Ioffe, The maximum principle in optimal control of systems governed by semilinear equations, Nonsmooth Analysis and Geometric Methods in Deterministic Optimal Control (B. S. Mordukhovich and H. J. Sussmann, eds.), IMA Volumes in Mathematics and its Applications, vol. 78, Springer, 1996, pp. 81-110.

20. A. D. Ioffe, Nonsmooth analysis: differential calculus of nondifferentiable mappings, Trans. Amer. Math. Soc. 266 (1981), 1-56. MR 82j:58018

21. A. D. Ioffe, Calculus of Dini subdifferentials and contingent derivatives of set-valued maps, Nonlinear Anal. 8 (1984), 517-539. MR 85k:46049

22. A. D. Ioffe, Approximate subdifferentials and applications. I: The finite dimensional theory, Trans. Amer. Math. Soc. 281 (1984), 389-416. MR 89m:49029

23. A. D. Ioffe, Approximate subdifferentials and applications. II: Functions on locally convex spaces, Mathematika 33 (1986), 111-128. MR 87k:49028

24. A. D. Ioffe, Approximate subdifferential and applications. III: The metric theory, Mathematika 36 (1989), 1-38. MR 90g:49012

25. A. D. Ioffe, Proximal analysis and approximate subdifferentials, J. London Math. Soc. 41 (1990), 175-192. MR 91i:46045

26. A. D. Ioffe, Nonsmooth subdifferentials: their calculus and applications, Proceedings of the First World Congress of Nonlinear Analysts, (V. Lakshmikantham, ed.), W. de Gruyter, Berlin, 1995.

27. A. D. Ioffe and R. T. Rockafellar, The Euler and Weierstrass conditions for nonsmooth variational problems, Calculus of Variations and PDEs (to appear).

28. A. Jofré, D. T. Luc, and M. Théra, $\varepsilon$-Subdifferential and $\varepsilon$-monotonicity, preprint, January 1995

29. A. Jourani and L. Thibault, A note of Fréchet and approximate subdifferentials of composite functions, Bull. Austral. Math. Soc. 49 (1994), 111-116. MR 95f:49019

30. A. Jourani and L. Thibault, Extensions of subdifferential calculus rules in Banach spaces and applications, Can. J. Math. (to appear).

31. A. Y. Kruger, Properties of generalized differentials, Siberian Math. J. 26 (1985), 822-832. MR 87d:90147

32. A. Y. Kruger, Generalized differentials of nonsmooth functions and necessary conditions for an extremum, Siberian Math. J. 26 (1985), 370-379. MR 86j:49038

33. A. Y. Kruger, A covering theorem for set-valued mappings, Optimization 19 (1988), 763-780. MR 89m: 49030

34. A. Y. Kruger and B. S. Mordukhovich, Extremal points and the Euler equation in nonsmooth optimization, Dokl. Akad. Nauk BSSR 24 (1980), 684-687. (Russian) MR 82b:90127

35. A. Y. Kruger and B. S. Mordukhovich, Generalized normals and derivatives, and necessary optimality conditions in nondifferentiable programming, Part I: Depon. VINITI, No. 408-80; Part II: Depon. VINITI, No. 494-80, Moscow, 1980. (Russian)

36. E. B. Leach, A note on inverse function theorem, Proc. Amer. Math. Soc. 12 (1961), 694-697. MR 23:A3442

37. P. D. Loewen, Proximal normal formula in Hilbert spaces, Nonlinear Anal. 11 (1987), 979-995. MR 89b: 49017

38. P. D. Loewen, Limits of Fréchet normals in nonsmooth analysis, Optimization and Nonlinear Analysis (A. D. Ioffe, L. Marcus, and S. Reich, eds.), Pitman Research Notes Math. Ser. 244, 1992, pp. 178-188. MR 93h:49028

39. P. D. Loewen, Optimal control via nonsmooth analysis, CRM Proceedings and Lecture Notes, Vol. 2, Amer. Math. Soc., Providence, RI, 1993. MR 94h:49003

40. P. D. Loewen, A mean value theorem for Fréchet subgradients, Nonlinear Anal. 23 (1994), 1365-1381. MR 95h:49023

41. B. S. Mordukhovich, Maximum principle in problems of time optimal control with nonsmooth constraints, J. Appl. Math. Mech. 40 (1976), 960 - 969. MR 58:7284

42. B. S. Mordukhovich, Metric approximations and necessary optimality conditions for general classes of nonsmooth extremal problems, Soviet Math. Dokl. 22 (1980), 526-530. MR 82b:90104

43. B. S. Mordukhovich, Nonsmooth analysis with nonconvex generalized differentials and adjoint mappings, Dokl. Akad. Nauk BSSR 28 (1984), 976-979. MR 86c:49018

44. B. S. Mordukhovich, Approximation methods in problems of optimization and control, "Nauka", Moscow, 1988. MR 89m:49001 
45. B. S. Mordukhovich, Sensitivity analysis in nonsmooth optimization, Theoretical Aspects of Industrial Design (D. A. Field and V. Komkov, eds.), SIAM Proc. Appl. Math., vol. 58, Soc. Indust. Appl. Math., Philadelphia, Pa., 1992, pp. 32-46. MR 93a:49012

46. B. S. Mordukhovich, Complete characterization of openness, metric regularity, and Lipschitzian properties of multifunctions, Trans. Amer. Math. Soc. 340 (1993), 1-35. MR 94a:49011

47. B. S. Mordukhovich, Lipschitzian stability of constraint systems and generalized equations, Nonlinear Anal. 22 (1994), 173-206. MR 94m:49041

48. B. S. Mordukhovich, Stability theory for parametric generalized equations and variational inequalities via nonsmooth analysis, Trans. Amer. Math. Soc. 343 (1994), 609-658. MR 94h: 49031

49. B. S. Mordukhovich, Generalized differential calculus for nonsmooth and set-valued mappings, J. Math. Anal. Appl. 183 (1994), 250-288. MR 95i:49029

50. B. S. Mordukhovich and Y. Shao, Differential characterizations of covering, metric regularity, and Lipschitzian properties of multifunctions between Banach spaces, Nonlinear Anal. 25 (1995), 1401-1424. CMP 96:02

51. B. S. Mordukhovich and Y. Shao, Extremal characterizations of Asplund spaces, Proc. Amer. Math. Soc. 124 (1996), 197-205.

52. B. S. Mordukhovich and Y. Shao, On nonconvex subdifferential calculus in Banach spaces, J. of Convex Analysis 2 (1995), 211-228

53. B. S. Mordukhovich and Y. Shao, Stability of set-valued mappings in infinite dimensions: point criteria and applications, SIAM J. Control Optim. (to appear).

54. J.-P. Penot, A mean value theorem with small subdifferentials, preprint, February 1995.

55. R. R. Phelps, Convex functions, monotone operators and differentiability, 2nd edition, Lecture Notes in Mathematics, vol. 1364, Springer, 1993. MR 94f:46055

56. R. Poliquin, Subgradient monotonicity and convex functions, Nonlinear Anal. 14 (1990), 305317. MR 91b:90155

57. D. Preiss, Differentiability of Lipschitz functions on Banach spaces, J. Func. Anal. 91 (1990), 312-345. MR 91g:46051

58. R. T. Rockafellar, Generalized directional derivatives and subgradients of nonconvex functions, Can. J. Math. 32 (1980), 257-280. MR 81f:49006

59. R. T. Rockafellar, Proximal subgradients, marginal values, and augmented Lagrangians in nonconvex optimization, Math. Oper. Res. 6 (1981), 424-436. MR 83m:90088

60. R. T. Rockafellar, Extensions of subgradient calculus with applications to optimization, Nonlinear Anal. 9 (1985), 665-698. MR 87a:90148

61. R. T. Rockafellar, Lipschitzian properties of multifunctions, Nonlinear Anal. 9 (1985), 867885. MR 87a:90149

62. R. T. Rockafellar, Maximal monotone relations and the second derivatives of nonsmooth functions, Ann. Inst. H. Poincaré Anal. Non Linéaire 2 (1985), 167-184. MR 87c:49021

63. R. T. Rockafellar and R. J-B Wets, Variational analysis, Springer (to appear).

64. L. Thibault, Subdifferentials of compactly Lipschitzian vector-valued functions, Ann. Mat. Pura Appl. 125 (1980), 157-192. MR 92h:58006

65. L. Thibault, On subdifferentials of optimal value functions, SIAM J. Control Optim. 29 (1991), 1019-1036. MR 92e:49027

66. L. Thibault, A note on the Zagrodny mean value theorem, preprint, July 1994.

67. J. S. Treiman, Clarke's gradients and epsilon-subgradients in Banach spaces, Trans. Amer. Math. Soc. 294 (1986), 65-78. MR 87d:90188

68. D. E. Ward and J. M. Borwein, Nonconvex calculus in finite dimensions, SIAM J. Control Optim. 25 (1987), 1312-1340. MR 88m:58011

69. J. Warga, Fat homeomorphisms and unbounded derivate containers, J. Math. Anal. Appl. 81 (1981), 545-560; errata, ibid 81 (1981), 582-583. MR 83f:58007; MR 84b:58016

70. D. Zagrodny, Approximate mean value theorem for upper subderivatives, Nonlinear Anal. 12 (1988), 1413-1428. MR 89k:58034

Department of Mathematics, Wayne State University, Detroit, Michigan 48202 E-mail address: boris@@math.wayne.edu 